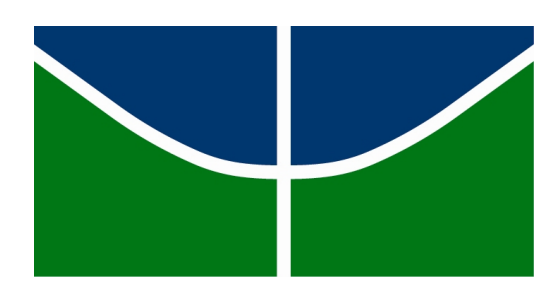

\author{
Universidade de Brasília \\ Instituto de Ciências Exatas \\ Departamento de Matemática
}

\title{
Equações elípticas semilineares e quasilineares com potenciais que mudam de sinal
}

\author{
por \\ José Carlos de Oliveira Junior
}


Universidade de Brasília

Instituto de Ciências Exatas

Departamento de Matemática

\section{Equações elípticas semilineares e quasilineares com potenciais que mudam de sinal}

por

\section{José Carlos de Oliveira Junior}

Tese apresentada ao Departamento de Matemática da Universidade de Brasília como parte dos requisitos necessários para obtenção do grau de

\section{DOUTOR EM MATEMÁTICA}

24 de setembro de 2015

Comissão Examinadora:

$\overline{\text { Profa. Dra. Liliane de Almeida Maia - Orientadora (MAT-UnB) }}$

$\overline{\text { Prof. Dr. Marcelo Fernandes Furtado (MAT-UnB) }}$

Prof. Dr. Ricardo Ruviaro (MAT-UnB)

Prof. Dr. Gaetano Siciliano (IME-USP)

$\overline{\text { Prof. Dr. Sérgio Henrique Monari Soares (ICMC-USP) }}$

*O autor foi bolsista CAPES e CNPq durante a elaboração deste trabalho. 
Aos meus pais,

José Carlos e Maria de Lourdes;

Aos meus irmãos,

Carolina e Pedro Henrique;

À minha amada noiva,

Renata. 
"... pois estive apoiado em ombros de gigantes". (I. Newton) 


\section{Agradecimentos}

- A Deus, por mais este sonho realizado. Neste período, pude entender um infinitésimo a mais do quanto Ele está próximo.

- Aos meus pais, José Carlos (mais do que em memória) e Maria de Lourdes, por valores ensinados a mim que vão durar por toda minha vida. À minha família, pelo apoio, referência e risadas, que tornaram tudo isso possível. Obrigado, minha amada avó Nacirema, pelo carinho e pelo acolhimento. Aos meus irmãos, Carolina e Pedro Henrique, pela força, apoio e gargalhadas de sempre.

- Aos amigos do poker, pelos momentos de ímpar descontração. À Ester, que foi tão gentil e cuidadosa comigo no período de minha chegada em Brasília. Aos amigos Maurílio e Gabriela, pelas boas e longas conversas. À Tânia, por estar perto, mesmo que distante, pelas valiosas e confortantes palavras nos momentos difíceis, pela amizade e pelo carinho de anos.

- A todos os amigos do departamento de matemática da UnB. Em especial, aos amigos Alireza, Somayeh, Ilana e Keidna. Agradeço por terem tornado esse período mais suave e "diferenciável".

- Aos funcionários da secretaria de graduação e pós-graduação. Destaco Marta e Bruna, pela amizade e pela presteza que exerceram na secretaria.

- Aos professores Marcelo Fernandes Furtado, Ricardo Ruviaro, Gaetano Siciliano e Sérgio Henrique Monari Soares, pela confiança e pelas valiosas sugestões que enriqueceram este trabalho.

- De uma forma especial, ao professor Ricardo Ruviaro, que foi mais do que um amigo neste período. Obrigado, professor, pelo apoio e força nos momentos complicados. 
- À minha querida orientadora, Liliane de Almeida Maia. Agradeço pela amizade, pela confiança, pelo cuidado, pela preocupação e pela paciência em responder minhas inúmeras dúvidas. Vou ser para sempre grato.

- À minha amada noiva, Renata Alves da Silva, pelo amor incondicional, pelas palavras de ânimo e por tornar minha vida mais prazerosa. Você foi essencial para que tudo isso acontecesse. Obrigado, amor!

- À CAPES e ao CNPq, pelo apoio financeiro durante a elaboração deste trabalho. 


\section{Resumo}

Neste trabalho, consideramos o problema autônomo

$$
-\Delta u+V(x) u=f(u) \text { em } \mathbb{R}^{N}
$$

com $u \in H^{1}\left(\mathbb{R}^{N}\right) \backslash\{0\}$, em que $N \geq 3$, a função $V$ é não periódica, radialmente simétrica e muda de sinal e a não linearidade $f$ é assintoticamente linear. Além disso, impomos que $V$ possui um limite positivo no infinito e que o espectro do operador $L=-\Delta+V$ tem ínfimo negativo. Sob essas condições, baseando-se em interações entre soluções transladadas do problema no infinito associado, é possível mostrar que tal problema satisfaz a geometria do teorema de linking clássico e garantir a existência de uma solução fraca não trivial.

Em seguida, estabelecemos a existência de uma solução não trivial para o problema não autônomo

$$
-\Delta u+V(x) u=f(x, u) \text { em } \mathbb{R}^{N},
$$

com $u \in H^{1}\left(\mathbb{R}^{N}\right) \backslash\{0\}$, sob hipóteses similares ao problema anterior, admitindo também que $f(x, u)=f(|x|, u)$ dentre outras condições. Aplicamos novamente o teorema de linking para garantir que tal problema possui uma solução não trivial.

Por fim, provamos que o problema quasilinear

$$
-\Delta u+V(x) u-u \Delta\left(u^{2}\right)=g(x, u) \text { em } \mathbb{R}^{3}
$$

com $u \in H^{1}\left(\mathbb{R}^{3}\right) \backslash\{0\}$, em que o potencial $V$ muda de sinal, podendo ser não limitado inferiormente, e a não linearidade $g(x, u)$, quando $|x| \rightarrow \infty$, tem um certo tipo de monotonicidade, possui uma solução não trivial. A existência de tal solução é provada por meio de uma mudança de variável que transforma o problema num problema semilinear, nos permitindo, assim, empregar o teorema do passo da montanha combinado com o lema splitting.

Palavras-chave: Assintoticamente linear, linking, teoria espectral, equações de Schrödinger não lineares, problemas fortemente indefinidos, equações quasilineares. 


\section{Abstract}

In this work, we consider the autonomous problem

$$
-\Delta u+V(x) u=f(u) \text { in } \mathbb{R}^{N},
$$

with $u \in H^{1}\left(\mathbb{R}^{N}\right) \backslash\{0\}$, where $N \geq 3, V$ is a non-periodic radially symmetric function that changes sign and the nonlinearity $f$ is asymptotically linear. Furthermore, we impose that $V$ has a positive limit at infinity and the spectrum of the operator $L=-\Delta+V$ has negative infimum. Under these conditions, employing interaction between translated solutions of the problem at infinity, it is possible to show that such problem satisfies the geometry of the classical linking theorem and garantee the existence of a nontrivial weak solution.

After that, we establish the existence of a nontrivial weak solution for the nonautonomous problem

$$
-\Delta u+V(x) u=f(x, u) \text { in } \mathbb{R}^{N},
$$

with $u \in H^{1}\left(\mathbb{R}^{N}\right) \backslash\{0\}$, under similar hyphoteses to the previous problem, assuming also that $f(x, u)=f(|x|, u)$ among others conditions. We apply again the classical linking theorem to ensure that such problem possesses a nontrivial weak solution.

Finally, we prove that the quasilinear problem

$$
-\Delta u+V(x) u-u \Delta\left(u^{2}\right)=g(x, u) \text { in } \mathbb{R}^{3},
$$

with $u \in H^{1}\left(\mathbb{R}^{3}\right) \backslash\{0\}$, where the potential $V$ changes sign and may be unbounded from below and the nonlinearity $g(x, u),|x| \rightarrow \infty$, has a kind of monotonicity, possesses a nontrivial weak solution. The existence of such solution is proved by means of a change of variables that makes the problem become a semilinear problem and hence allow us apply the mountain pass theorem combined with splitting lemma.

Keywords: Asymptotically linear, linking theorem, spectral theory, nonlinear Schrödinger equations, strongly indefinite problem, quasilinear equations. 


\section{Sumário}

1 Espectro de um operador auto-adjunto e propriedades das autofunções 4

1.1 O espectro de um operador auto-adjunto $S \ldots \ldots \ldots \ldots$

1.2 Propriedades das autofunções . . . . . . . . . . . . . . . 9

2 Existência de solução para um problema fortemente indefinido $\quad 11$

2.1 Estrutura variacional do problema . . . . . . . . . . . . . . 14

2.2 Limitação das sequências de Cerami . . . . . . . . . . . . . . . 15

2.3 Uma solução não trivial . . . . . . . . . . . . . . . . . . 22

$3 \quad$ Um problema não autônomo fortemente indefinido 35

3.1 Estrutura variacional do problema . . . . . . . . . . . . 37

3.2 Limitação das sequências de Cerami . . . . . . . . . . . . . . . 38

3.3 Uma solução não trivial . . . . . . . . . . . . . . . . . . . . . . 44

4 Uma equação de Schrödinger quasilinear com um potencial indefinido 49

4.1 Resultados principais . . . . . . . . . . . . . . . 51

5 Apêndice A $\quad 63$

$\begin{array}{ll}\text { Referências Bibliográficas } & 66\end{array}$ 


\section{Introdução}

Nosso trabalho está estruturalmente dividido em quatro capítulos. No primeiro capítulo, vamos recordar noções de Análise Funcional e apresentar uma pequena parte da Teoria Espectral no que diz respeito a operadores auto-adjuntos, espectro, autovalores e autofunções de um operador linear. O ponto de partida para o segundo capítulo é a equação de Schrödinger não linear com um potencial que possui parte negativa. Nosso objetivo é considerar esta classe de problemas e mostrar a existência de uma solução não trivial para

$$
-\Delta u+V(x) u=f(u) \quad \text { em } \mathbb{R}^{N}
$$

em que $N \geq 3$, com potencial contínuo não periódico $V$ que muda de sinal e possui limite assintótico $V_{\infty}>0$ no infinito e com $f$ uma função assintoticamente linear no infinito. Muitos autores têm estudado o problema $(A)$ com vários tipos de potenciais $V$ e não linearidades $f$.

Kryszewski e Szulkin em [27], Pankov em [35] e Pankov e Pflüger em [36], provaram a existência de uma solução não trivial para o problema não autônomo, com $f(x, u)$ em $(A)$, admitindo $f(x, s)$ superquadrática em $s$ e hipóteses de periodicidade. Recentemente, Szulkin e Weth em [47] estudaram existência e multiplicidade de soluções para o caso não autônomo desde que $V$ e $f$ sejam periódicas em $x$ e $s \mapsto f(x, s) /|s|$ seja estritamente crescente em $(-\infty, 0)$ e em $(0, \infty)$. Sob condições similares, Furtado, Maia e Medeiros em [22] provaram a existência de uma solução positiva e de uma solução nodal usando o método de Nehari.

Para $f$ assintoticamente linear no infinito, Jeanjean e Tanaka em [25] estabeleceram a existência de uma solução positiva para $(A)$ no caso definido, $V(x) \geq \alpha>0$.

Em 2006, Liu, Su e Weth em [32] estudaram o problema não autônomo sem hipótese de periodicidade para as funções $V$ e $f$ e provaram a existência de soluções positiva, negativa e nodal para o problema $(A)$.

O problema autônomo $(A)$ foi considerado por Azzollini e Pomponio em [3] sob as condições de Berestycki-Lions na função $f$ e $V$ uma função de classe $C^{1}$, radialmente simétrica, que possui uma limitação técnica na parte positiva de seu gradiente. Eles 
provaram a existência de uma solução radialmente simétrica para o problema $(A)$.

Estamos interessados em estudar o problema $(A)$ em que $V$ é não periódica, muda de sinal e possui limite assintótico $V_{\infty}$ no infinito e $f$ é assintoticamente linear no infinito. Uma dificuldade encontrada em estudar problemas deste tipo é que o funcional associado $I$ é fortemente indefinido. É conveniente, então, decompor o espaço $H^{1}\left(\mathbb{R}^{N}\right)$ em uma soma direta de dois subespaços, $E^{+}$e $E^{-}$, um deles de dimensão finita, e assumir a condição de não quadraticidade em $F$, a primitiva de $f$. Sob certas condições no potencial $V$ e na função $f$, provamos o resultado principal do segundo capítulo, que garante a existência de uma solução não trivial para o problema $(A)$.

O terceiro capítulo é dedicado a estabelecer a existência de uma solução não trivial para o problema elíptico

$$
-\Delta u+V(x) u=f(x, u) \quad \text { em } \mathbb{R}^{N},
$$

sem condições de periodicidade em $V$ e $f$, onde o potencial $V$ muda de sinal e a não linearidade $f$ é assintoticamente linear no infinito.

Problemas desse tipo tem sido estudado extensivamente para $V$ e $f$ periódicas na variável $x$, a fim de suprir a falta de compacidade em $\mathbb{R}^{N}$ (veja $[24,27,28,36,47]$ e suas referências).

A respeito de problemas assintoticamente lineares, Stuart e Zhou no trabalho [46] assumiram $V$ constante e $f$ radialmente simétrica em $x$ e foram capazes de mostrar a existência de uma solução positiva radialmente simétrica para o problema $(N A)$.

Assim como no capítulo anterior, embora não seja possível aplicar o teorema do passo da montanha, fazemos uso do teorema de linking clássico [38] sob a condição de Cerami [6], a fim de obter uma solução não trivial para o problema $(N A)$, admitindo certas hipóteses no potencial $V$ e na não linearidade $f$.

Nosso objetivo no quarto capítulo é mostrar a existência de uma solução estacionária não trivial da seguinte equação de Schrödinger quasilinear

$$
-\Delta u+V(x) u-u \Delta\left(u^{2}\right)=g(x, u) \quad \text { em } \mathbb{R}^{3},
$$

onde $g$ tem um crescimento subcrítico e $V$ é um potencial que muda de sinal, mas não é necessariamente simétrico, podendo ser ilimitado inferiormente.

Existem muitos trabalhos sobre o problema $(Q)$ com $V$ satisfazendo $\inf _{\mathbb{R}^{N}} V(x)>0$ e $g$ uma não linearidade que possui crescimento subcrítico ou crítico (veja, por exemplo, $[8,21,31,37,41,42,48])$. O caso de massa zero, $V \equiv 0$, foi estudado em [8]. O caso em que o potencial converge a zero foi considerado em [1].

Outro trabalho recente em equações de Schrödinger quasilineares com potencial que 
muda de sinal é [20], o qual estuda o problema $(Q)$ sob hipóteses muito similares às nossas. Todavia, a existência de uma solução fraca não trivial para o problema não autônomo é garantida acrescentando-se um termo de perturbação não trivial a não linearidade, o que trivializa os argumentos dos autores, pois os mesmos não precisam lidar com questões de compacidade.

Para provarmos o resultado principal do quarto capítulo, ou seja, a existência de uma solução não trivial para a equação $(Q)$, usaremos métodos variacionais combinados com o princípio de concentração e compacidade de Lions [30]. A fim de lidar com o potencial $V$ que muda de sinal, fomos beneficiados com algumas ideias de [22].

O Apêndice A contém resultados técnicos e suas demonstrações, que serão utilizados no segundo e no terceiro capítulos deste trabalho. 


\section{Capítulo 1}

\section{Espectro de um operador auto-adjunto e propriedades das autofunções}

Neste capítulo, vamos recordar noções de Análise Funcional e apresentar uma pequena parte da teoria espectral no que diz respeito a operadores auto-adjuntos, espectro, autovalores e autofunções de um operador linear. O desenvolvimento deste capítulo está baseado num trabalho sobre teoria espectral para operadores auto-adjuntos de Schrödinger [44].

\subsection{O espectro de um operador auto-adjunto $S$}

Começamos recordando definições básicas concernentes a operadores auto-adjuntos. Seja $(H,\langle\rangle$,$) um espaço de Hilbert sobre \mathbb{R}$.

Definição 1 Seja $L: D(L) \subset H \rightarrow H$ um operador linear cujo dominio $D(L)$ é um subespaço denso de $H$. O operador adjunto de $L, L^{*}: D\left(L^{*}\right) \subset H \rightarrow H$, é definido como segue:

$$
v \in D\left(L^{*}\right) \Longleftrightarrow\left\{\begin{array}{c}
v \in H \text { e existe um elemento } w \in H \\
\text { tal que }\langle L(u), v\rangle=\langle u, w\rangle \text { para todo } u \in D(L) .
\end{array}\right.
$$

e $L^{*} v=w$ para todo $v \in D\left(L^{*}\right)$, onde $w$ é o (único, pela densidade de $D(L)$ em $H$ ) elemento associado a $v$ na definição de $D\left(L^{*}\right)$. Dizemos que um operador $L$ é autoadjunto se $L=L^{*}$, isto é, $D(L)=D\left(L^{*}\right)$ e $L^{*} v=L v$ para todo $v \in D\left(L^{*}\right)$. 
Definição 2 Dado $V \in L^{\infty}\left(\mathbb{R}^{N}\right)$, definimos o operador de Schrödinger $S: D(S) \subset$ $L^{2}\left(\mathbb{R}^{N}\right) \rightarrow L^{2}\left(\mathbb{R}^{N}\right)$ gerado pelo potencial $V$ por

$$
D(S)=H^{2}\left(\mathbb{R}^{N}\right) \quad \text { e } \quad S u=-\Delta u+V u \quad \text { para } \quad u \in H^{2}\left(\mathbb{R}^{N}\right) .
$$

O primeiro resultado sobre operadores de Schrödinger é o seguinte.

Teorema 3 Para $V \in L^{\infty}\left(\mathbb{R}^{N}\right)$, o operador de Schrödinger $S: D(S) \subset L^{2}\left(\mathbb{R}^{N}\right) \rightarrow$ $L^{2}\left(\mathbb{R}^{N}\right)$ gerado pelo potencial $V$ é auto-adjunto.

Demonstração. Uma vez que $H^{2}\left(\mathbb{R}^{N}\right)$ é denso em $L^{2}\left(\mathbb{R}^{N}\right)$, o operador adjunto $S^{*}$ : $D\left(S^{*}\right) \subset L^{2}\left(\mathbb{R}^{N}\right) \rightarrow L^{2}\left(\mathbb{R}^{N}\right)$ está bem definido. Além disso, para toda função $u, v \in$ $H^{2}\left(\mathbb{R}^{N}\right)$, segue diretamente da definição de derivada fraca e de argumentos de densidade que

$$
\int_{\mathbb{R}^{N}}(S u) v d x=\int_{\mathbb{R}^{N}}(-\Delta u+V(x) u) v d x=\int_{\mathbb{R}^{N}} u(-\Delta v+V(x) v) d x
$$

onde $-\Delta v+V(x) v \in L^{2}\left(\mathbb{R}^{N}\right)$. Isto garante que $H^{2}\left(\mathbb{R}^{N}\right) \subset D\left(S^{*}\right)$ e que $S^{*}(v)=$ $-\Delta v+V(x) v=S v$ para todo $v \in H^{2}\left(\mathbb{R}^{N}\right)$. Para completar a prova do teorema, resta mostrar que $D\left(S^{*}\right) \subset H^{2}\left(\mathbb{R}^{N}\right)$. Ora, se $v \in D\left(S^{*}\right)$, então $v \in L^{2}\left(\mathbb{R}^{N}\right)$ e existe um elemento único $w \in L^{2}\left(\mathbb{R}^{N}\right)$ tal que

$$
\int_{\mathbb{R}^{N}}(S u) v d x=\int_{\mathbb{R}^{N}} u w d x \quad \text { para todo } u \in D(S)=H^{2}\left(\mathbb{R}^{N}\right) \subset L^{2}\left(\mathbb{R}^{N}\right) .
$$

Assim,

$$
\int_{\mathbb{R}^{N}} v(\Delta u) d x=\int_{\mathbb{R}^{N}}(V(x) v-w) u d x \quad \text { para todo } u \in H^{2}\left(\mathbb{R}^{N}\right) .
$$

Pelo Lema 2.11 em [44], $v \in H^{2}\left(\mathbb{R}^{N}\right)$, concluindo a prova do teorema.

Definição 4 Seja $L: D(L) \subset H \rightarrow H$ um operador auto-adjunto. Definimos o resolvente de $L$ como o conjunto

$$
\rho(L)=\{\lambda \in \mathbb{R} ; L-\lambda I: D(L) \rightarrow H \text { é um isomorfismo }\}
$$

e o espectro de L como o conjunto

$$
\sigma(L)=\mathbb{R} \backslash \rho(L)
$$

Os elementos de $\rho(L)$ são chamados valores regulares de L. O espectro pontual de L é o conjunto

$$
\sigma_{p}(L)=\{\lambda \in \mathbb{R} ; \operatorname{ker}(L-\lambda I) \neq\{0\}\}
$$


e seus elementos são chamados autovalores de L. O espectro discreto de L é o conjunto

$$
\sigma_{d}(L)=\left\{\lambda \in \sigma_{p}(L) ; \operatorname{dim} \operatorname{ker}(L-\lambda I)<\infty \text { e } \lambda \text { é um ponto isolado de } \sigma_{p}(L)\right\}
$$

e seu complemento em $\sigma(L)$ é chamado espectro essencial de $L$,

$$
\sigma_{e}(L)=\sigma(L) \backslash \sigma_{d}(L)
$$

Observe que, por definição, os elementos de $\sigma_{d}(L)$ são autovalores isolados de $L$ de multiplicidade finita. Os conjuntos definidos acima desempenham um papel fundamental na teoria de operadores auto-adjuntos. Não somente eles, mas também o número

$$
\Lambda=\inf \left\{\int_{\mathbb{R}^{N}}\left(|\nabla u|^{2}+V(x) u^{2}\right) d x ; u \in H^{1}\left(\mathbb{R}^{N}\right) \text { e } \int_{\mathbb{R}^{N}} u^{2} d x=1\right\}
$$

para $V \in L^{\infty}\left(\mathbb{R}^{N}\right)$. Uma vez que $|V(x)| \leq\|V\|_{L^{\infty}\left(\mathbb{R}^{N}\right)}$, tem-se

$$
\int_{\mathbb{R}^{N}}\left(|\nabla u|^{2}+V(x) u^{2}\right) d x \geq \int_{\mathbb{R}^{N}} V(x) u^{2} d x \geq-\|V\|_{L^{\infty}\left(\mathbb{R}^{N}\right)} \int_{\mathbb{R}^{N}} u^{2} d x
$$

para qualquer $u \in H^{1}\left(\mathbb{R}^{N}\right)$. Isso mostra que $\Lambda \geq-\|V\|_{L^{\infty}\left(\mathbb{R}^{N}\right)}$.

O teorema a seguir mostra que o espectro do operador $S$ nunca é vazio e, além disso, caracteriza seu ínfimo, relacionando-o com o número $\Lambda$.

Teorema 5 Seja $V \in L^{\infty}\left(\mathbb{R}^{N}\right)$. Então,

$$
\text { i) } \sigma(S) \subset[\Lambda,+\infty) \quad e \quad \text { ii) } \Lambda \in \sigma(S)
$$

Em particular, $\Lambda=\inf \sigma(S)$.

A demonstração deste resultado se baseia em dois lemas, os quais enunciamos abaixo.

Lema 6 Seja $L: D(L) \subset H \rightarrow H$ um operador auto-adjunto definido num espaço de Hilbert $H$ sobre $\mathbb{R}$. Para $\lambda \in \mathbb{R}$, temos que

$$
L-\lambda I: D(L) \subset H \rightarrow H \quad \text { é um isomorfismo }
$$

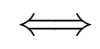

Existe $c>0$ tal que $\|(L-\lambda I) u\| \geq c\|u\|$ para todo $u \in D(L)$. 
Demonstração. Veja Lema 3.2 em [44].

Lema 7 Seja $V \in L^{\infty}\left(\mathbb{R}^{N}\right)$. Então,

(1) também temos

$$
\Lambda=\inf \left\{\int_{\mathbb{R}^{N}}(S u) u d x ; u \in H^{2}\left(\mathbb{R}^{N}\right) e \int_{\mathbb{R}^{N}} u^{2} d x=1\right\}
$$

(2) se $u \in H^{1}\left(\mathbb{R}^{N}\right)$ com $\int_{\mathbb{R}^{N}} u^{2} d x=1 e \int_{\mathbb{R}^{N}}\left(|\nabla u|^{2}+V(x) u^{2}\right) d x=\Lambda$, então

$$
u \in H^{2}\left(\mathbb{R}^{N}\right), u \in \operatorname{ker}(S-\Lambda I) \quad \text { e } \Lambda \in \sigma_{p}(S) .
$$

Demonstração. Veja Lema 3.9 em [44].

Prova do Teorema 5 Vamos provar $(i)$. Pelo Lema 7 parte $(1)$, para todo $u \in H^{2}\left(\mathbb{R}^{N}\right)$,

$$
\Lambda \int_{\mathbb{R}^{N}} u^{2} d x \leq \int_{\mathbb{R}^{N}}(S u) u d x
$$

Portanto, dado $\lambda \in \mathbb{R}$, somando $-\lambda \int_{\mathbb{R}^{N}} u^{2} d x$ na desigualdade acima, segue da desigualdade de Hölder que

$$
(\Lambda-\lambda) \int_{\mathbb{R}^{N}} u^{2} d x \leq \int_{\mathbb{R}^{N}}\{(S u-\lambda I) u\} u d x \leq\|(S-\lambda I) u\|_{L^{2}\left(\mathbb{R}^{N}\right)}\|u\|_{L^{2}\left(\mathbb{R}^{N}\right)} .
$$

Assim,

$$
\|(S-\lambda I) u\|_{L^{2}\left(\mathbb{R}^{N}\right)}\|\geq(\Lambda-\lambda)\| u \|_{L^{2}\left(\mathbb{R}^{N}\right)} \text { para todo } u \in D(S) .
$$

Segue do Lema 6 que $\lambda \in \rho(S)$ se $\Lambda-\lambda>0$. Isso conclui a demonstração de $(i)$. Para provar que (ii) é válido, pela parte $(i)$, sabemos que $\sigma(S) \subset[\Lambda,+\infty)$. Uma vez que $\sigma(S)$ é um conjunto fechado, seja $m \geq \Lambda$ tal que $\sigma(S) \subset[m,+\infty)$ e vamos mostrar que com isso $m \leq \Lambda$. Escolha $\xi \in(-\infty, m)$. Desde que $\xi \in \rho(S)$, podemos tomar

$$
A:=(S-\xi I)^{-1}
$$

tal que

$$
A: L^{2}\left(\mathbb{R}^{N}\right) \rightarrow H^{2}\left(\mathbb{R}^{N}\right) \subset L^{2}\left(\mathbb{R}^{N}\right) \text { é um operador linear contínuo e auto-adjunto. }
$$


Uma vez que $H^{2}\left(\mathbb{R}^{N}\right) \neq L^{2}\left(\mathbb{R}^{N}\right)$, o operador $A$ não é sobrejetivo e, assim, $0 \in \sigma(A)$. Para $\lambda \neq 0$,

$$
A-\lambda I=\lambda\left(\frac{1}{\lambda} I-(S-\xi I)\right) A=\lambda\left\{\left(\frac{1}{\lambda}+\xi\right) I-S\right\} A .
$$

Assim, $A-\lambda I: L^{2}\left(\mathbb{R}^{N}\right) \rightarrow L^{2}\left(\mathbb{R}^{N}\right)$ é um isomorfismo se, e somente se, $S-\left(\frac{1}{\lambda}+\xi\right) I: H^{2}\left(\mathbb{R}^{N}\right) \rightarrow L^{2}\left(\mathbb{R}^{N}\right)$ é um isomorfismo, isto é, se, e somente se, $\left(\frac{1}{\lambda}+\xi\right) \in \rho(S)$. Isto nos diz que, se $\lambda \in \rho(A)$, então $\lambda=\frac{1}{\mu-\xi}$ para algum $\mu \in \rho(S)$. Portanto, podemos escrever

$$
\sigma(A)=\{0\} \cup\left\{\frac{1}{\mu-\xi} ; \mu \in \rho(S)\right\}
$$

e, consequentemente,

$$
\sigma(A) \subset\left[0, \frac{1}{m-\xi}\right] .
$$

Pelo Lema 3.4 de [44], isto implica que

$$
\int_{\mathbb{R}^{N}}(A v) v d x \geq 0 \text { para todo } v \in L^{2}\left(\mathbb{R}^{N}\right) .
$$

Para cada $u \in H^{2}\left(\mathbb{R}^{N}\right)$, seja $v=(S-\xi I) u$. Então,

$$
\int_{\mathbb{R}^{N}}[(S-\xi I) u] u d x=\int_{\mathbb{R}^{N}}(A v) v d x \geq 0 .
$$

Isto mostra que $\int_{\mathbb{R}^{N}}(S u) u d x \geq \xi \int_{\mathbb{R}^{N}} u^{2} d x$ para todo $u \in H^{2}\left(\mathbb{R}^{N}\right)$ e segue do Lema 7 parte (2) que $\xi \leq \Lambda$. Pela arbitrariedade de $\xi$, temos $m \leq \Lambda$, completando a prova do teorema.

Assim como o Teorema 5 caracteriza o ínfimo do espectro do operador $S$, em certas condições, é possível caracterizar o ínfimo do espectro essencial do operador $S$. Para isso, considere

$$
V_{\infty}:=\lim _{R \rightarrow+\infty} V(x)
$$

O resultado a seguir mostra a importância do número $V_{\infty}$ devido à sua relação com o espectro essencial do operador $S$.

Teorema 8 Seja $V \in L^{\infty}\left(\mathbb{R}^{N}\right)$ satisfazendo (1.2). Então, $\sigma_{e}(S)=\left[V_{\infty},+\infty\right)$. 
Demonstração. Veja Teorema 3.15 em [44].

Observe que, pelo Teorema $8, \sigma_{d}(S) \neq \emptyset$ se, e somente se, $\Lambda<V_{\infty}$.

\subsection{Propriedades das autofunções}

Procuramos obter propriedades para as soluções da equação $S u=\lambda u$, para algum $\lambda \in \mathbb{R}$. A primeira delas é sobre regularidade.

Teorema 9 Seja $V \in L^{\infty}\left(\mathbb{R}^{N}\right)$ e considere $u \in \operatorname{ker}(S-\lambda I)$ para algum $\lambda \in \mathbb{R}$. Então,

$$
u \in C\left(\mathbb{R}^{N}\right) \cap H^{2}\left(\mathbb{R}^{N}\right) \cap W^{1, s}\left(\mathbb{R}^{N}\right) \quad \text { para } \quad 2 \leq s \leq+\infty \quad e \quad \lim _{|x| \rightarrow+\infty} u(x)=0 .
$$

Demonstração. A demonstração se baseia no argumento de bootstrap, considerando $g(x)=\lambda u-V(x) u$. Veja Teorema 3.18 em [44].

A seguinte propriedade mostra informações sobre o decaimento das autofunções do operador $S$.

Teorema 10 Seja $V \in L^{\infty}\left(\mathbb{R}^{N}\right)$ e escolha $\xi<V_{\infty}$. Para qualquer $\eta \in\left(0, \sqrt{V_{\infty}-\xi}\right)$, existe uma constante $C>0$, dependendo apenas de $\xi$ e $\eta$, tal que

$$
|u(x)| \leq C\|u\|_{\infty} e^{-\eta|x|}, \quad \text { para todo } \quad x \in \mathbb{R}^{N}
$$

desde que $u \in \operatorname{ker}(S-\lambda u)$, para algum $\lambda \leq \xi$.

Demonstração. Veja Teorema 3.19 em [44].

O seguinte resultado mostra provavelmente a mais importante propriedade do número $\Lambda$.

Teorema 11 Seja $V \in L^{\infty}\left(\mathbb{R}^{N}\right)$ com $\Lambda<V_{\infty}$, onde $\Lambda$ e $V_{\infty}$ estão definidos em (1.1) e (1.2), respectivamente. Então, existe um elemento $\psi \in C\left(\mathbb{R}^{N}\right) \cap H^{2}\left(\mathbb{R}^{N}\right)$ tal que

$$
\psi(x)>0 \quad \text { para todo } \quad x \in \mathbb{R}^{N} \quad e \quad \operatorname{ker}(S-\Lambda I)=\operatorname{span}\{\psi\}
$$

Demonstração. Ver Teorema 3.20 em [44].

Problemas fortemente indefinidos são caracterizados por $\Lambda<0$. Daremos exemplos de condições suficientes na função $V$ para que isto ocorra. Ora, queremos encontrar uma 
função teste $u \in H^{1}\left(\mathbb{R}^{N}\right)$ tal que $\int_{\mathbb{R}^{N}}\left(|\nabla u|^{2}+V(x) u^{2}\right) d x<0$. Quando $N=1$ ou 2 e $V$ é uma função contínua com $V(x)<0$, isto é sempre possível (veja [39], Teorema XIII.77). Para $N \geq 3$, considere a equação diferencial de Bessel

$$
t^{2} y^{\prime \prime}(t)+t y^{\prime}(t)+\left(t-\alpha^{2}\right) y=0
$$

$\operatorname{com} \alpha, t \in \mathbb{R}$. É provado que existem duas soluções linearmente independentes para esta equação. Por meio de série de potências, obtém-se uma dessas soluções, chamada de função de Bessel do primeiro tipo e denotada por $J_{\alpha}$. Seja $t_{n}$ a menor raiz positiva da função $J_{(4-n) / 2}$ e considere

$$
V(x) \leq \begin{cases}-T & \forall|x|<R \\ 0 & \forall|x| \geq R\end{cases}
$$

com $R^{2} T>t_{n}$. Sob essas condições, vale $\Lambda<0$ (veja [16], página 283). 


\section{Capítulo 2}

\section{Existência de solução para um problema fortemente indefinido}

Uma equação de Schrödinger não linear que modela a propagação de um feixe de luz em um certo meio pode apresentar um potencial que muda de sinal no termo linear e determinar uma equação elíptica semilinear em $\mathbb{R}^{N}$ com um potencial que possui parte negativa (veja [45] e suas referências). Nosso objetivo é considerar esta classe de problemas e mostrar a existência de uma solução não trivial para

$$
-\Delta u+V(x) u=f(u) \quad \text { em } \mathbb{R}^{N}
$$

$N \geq 3$, com potencial contínuo não periódico $V$ que muda de sinal e possui limite assintótico $V_{\infty}>0$ no infinito e com $f$ sendo uma função assintoticamente linear no infinito.

Kryszewski e Szulkin em [27] provaram a existência de uma solução não trivial para o problema não autônomo, com $f(x, u)$ em $(P)$, sob a condição de 0 pentencer a um gap do espectro do operador $-\Delta+V$ assumindo que $V$ é um potencial periódico e $f(x, s)$ é superquadrática em $s$. A prova deles se baseia na decomposição de $H^{1}\left(\mathbb{R}^{N}\right)$ em dois subespaços de dimensão infinita e no teorema de linking generalizado, o qual é aplicado ao funcional energia associado ao problema. Este teorema de linking generalizado requer a construção de uma nova teoria do grau para suprir a falta de compacidade das imersões de Sobolev em $\mathbb{R}^{N}$.

O caso não autônomo também foi estudado por Pankov em [35] e por Pankov e Pflüger em [36]. Eles provaram a existência de uma solução não trivial desde que $V$ e $f$ sejam funções periódicas em $x$ e a não linearidade $f$ satisfaça a condição de AmbrossetiRabinowitz. Os autores consideraram uma sequência de problemas aproximados e provaram um teorema geral que garante a existência de um limite não trivial. Eles aplicaram 
argumentos da variedade de Nehari generalizada e o teorema de linking aos problemas aproximados conseguindo uma sequência de soluções periódicas, a qual converge a uma solução do problema não autônomo em $\mathbb{R}^{N}$. Recentemente, Szulkin e Weth em [47] estudaram existência e multiplicidade de soluções para o caso não autônomo desde que $V$ e $f$ sejam periódicas em $x, s \mapsto f(x, s) /|s|$ seja estritamente crescente em $(-\infty, 0)$ e em $(0, \infty)$ e $F$, a primitiva de $f$, seja superquadrática no infinito. Destacamos que esta última hipótese é crucial para que os autores demonstrem seus resultados. Eles utilizaram o método de minimização sobre a variedade de Nehari generalizada, introduzida por Pankov em [35]. Já Évéquoz e Weth em [19] aplicaram este método para encontrar uma solução no caso em que $V$ é não periódica e pode mudar de sinal, tem limite no infinito, a não linearidade $F$ é superquadrática no inifinito e a função $s \mapsto f(s) / s$ é estritamente crescente. Sob condições similares, Furtado, Maia e Medeiros em [22] provaram a existência de uma solução positiva e de uma solução nodal usando o método de Nehari.

Uma solução positiva radial foi obtida por Stuart e Zhou em [46], onde o problema é radialmente simétrico, o potencial $V$ é constante e $s \mapsto f(|x|, s) / s$ é não decrescente em $s \in(0, \infty)$. Em [24], Jeanjean obteve uma solução positiva, assumindo $V$ uma constante, $f(x, s)$ periódica em $x$ e $s \mapsto F(x, s) / s^{2}$ não decrescente em $s \in(0, \infty)$. Li e Szulkin em [28] também estudaram o problema não autônomo com $V$ e $f$ funções periódicas, considerando uma função auxiliar $g$ tal que $s \mapsto g(x, s) / s$ é estritamente crescente para $s>0$ e estritamente decrescente para $s<0$.

Outrossim, Jeanjean e Tanaka em [25] estabeleceram a existência de uma solução positiva para $(P)$ no caso definido, $V(x) \geq \alpha>0$, e com $f$ assimtoticamente linear no infinito, $f(s) s^{-1} \rightarrow a>0$ quando $s \rightarrow+\infty$, com $a>\inf \sigma(-\Delta+V)$, onde $\sigma(-\Delta+V)$ denota o espectro do operador auto-adjunto $-\Delta+V$.

Em 2006, Liu, Su e Weth em [32] estudaram o problema não autônomo sem hipótese de periodicidade para as funções $V$ e $f$ e provaram a existência de soluções positiva, negativa e nodal sob condições bem técnicas que colocam o problema dentro das condições do teorema do passo da montanha. Ding e Ruf em [14] provaram a existência e multiplicidade de soluções para uma equação de Dirac em $\mathbb{R}^{3}$, admitindo $\lim _{|s| \rightarrow \infty} f(x, s) / s:=m<\beta$, onde $\beta$ é o limite superior do gap espectral, e $f(x, s)$ é uma não linearidade assintoticamente quadrática sem hipótese alguma sobre periodicidade. Entre outras condições, a hipótese $m<\beta$ garante a convergência das sequências de Cerami, dada em [14], Lema 3.14. Todavia, esta hipótese não é satisfeita nesse trabalho, uma vez que $m>V_{\infty} \geq \beta$ pelas condições $\left(V_{2}\right),\left(V_{3}\right)$ e $\left(f_{2}\right)$ abaixo.

O problema autônomo $(P)$ foi estudado por Azzollini e Pomponio em [3] sob as condições de Berestycki-Lions na função $f$. No trabalho deles, $V$ é uma função de classe $C^{1}$, radialmente simétrica que possui uma limitação técnica na parte positiva de seu gradi- 
ente. Eles provaram a existência de uma solução radialmente simétrica para o problema $(P)$.

Estamos interessados em estudar o problema $(P)$ em que $V$ não periódica, muda de sinal e possui limite assintótico $V_{\infty}$ no infinito e $f$ é assintoticamente linear no infinito e satisfaz $s \mapsto f(s) / s$ é estritamente crescente em $s \in(0,+\infty)$. Aplicamos o teorema de linking clássico [38] com a condição de Cerami como em [29], Proposição 2.10, e [6]. Isto foi possível utilizando uma solução ground state positiva $u_{0}$ do problema limite

$$
-\Delta w+V_{\infty} w=f(w), \text { em } \mathbb{R}^{N}
$$

projetada em um subespaço vetorial de dimensão infinita, que possui codimensão finita, contido em $H^{1}\left(\mathbb{R}^{N}\right)$. Além disso, foi crucial estimar as interações das transladadas de $u_{0}$ a fim de obter a geometria de linking (veja Lema 17).

Uma dificuldade encontrada em estudar problemas deste tipo é que o funcional associado $I$ é fortemente indefinido. É conveniente, então, decompor o espaço $H^{1}\left(\mathbb{R}^{N}\right)$ em uma soma direta de dois subespaços, $E^{+}$e $E^{-}$, um deles de dimensão finita, e assumir a condição de não quadraticidade em $F$ (veja hipótese $\left(f_{3}\right)$ ).

Até onde entendemos, nossos resultados são originais no que se refere à existência de uma solução não trivial para $(P)$ sem hipóteses de periodicidade em $V$, um potencial que muda de sinal, e uma não linearidade $f$ assintoticamente linear no infinito.

Consideraremos o problema elíptico $(P) \operatorname{com} N \geq 3, u \in E:=H^{1}\left(\mathbb{R}^{N}\right)$ e $V$ um potencial satisfazendo as seguintes condições:

$\left(V_{1}\right) V \in C\left(\mathbb{R}^{N}, \mathbb{R}\right)$ e $-V_{0} \leq V(x) \leq V_{\infty}$, em que $V_{0}, V_{\infty}>0 ;$

$\left(V_{2}\right) \lim _{|x| \rightarrow+\infty} V(x)=V_{\infty} ;$

$\left(V_{3}\right) 0 \notin \sigma(L)$ e $\inf \sigma(L)<0$, onde $\sigma(L)$ é o espectro do operador $L=-\Delta+V$;

$\left(V_{4}\right) V(x) \leq V_{\infty}-C_{1} e^{-\gamma|x|}$, em que $C_{1}>0$ e $0<\gamma<\sqrt{V_{\infty}}$.

As condições que impomos sobre a não linearidade $f$ são:

$\left(f_{1}\right) f \in C^{3}(\mathbb{R}, \mathbb{R})$ e $\lim _{s \rightarrow 0} \frac{f(s)}{s}=0 ;$

$\left(f_{2}\right) \lim _{|s| \rightarrow+\infty} \frac{|f(s)|}{|s|}=m>V_{\infty} \quad$ e $\quad \frac{|f(s)|}{|s|}<m$ para todo $s \in \mathbb{R} \backslash\{0\} ;$

$\left(f_{3}\right)$ Se $F(s):=\int_{0}^{s} f(t) d t$ e $Q(s):=\frac{1}{2} f(s) s-F(s)$, então, para todo $s \in \mathbb{R} \backslash\{0\}$,

$$
F(s) \geq 0, \quad Q(s)>0 \quad \text { e } \quad \lim _{s \rightarrow \infty} Q(s)=+\infty .
$$


$\left(f_{4}\right)$ Existem $C_{2}>0$ e $1<p_{1} \leq p_{2}$ tais que $p_{1}, p_{2}<2^{*}-1$ e

$$
\left|f^{(k)}(s)\right| \leq C_{2}\left(|s|^{p_{1}-k}+|s|^{p_{2}-k}\right)
$$

para $k \in\{0,1,2,3\}$ e $s \in \mathbb{R}$

$\left(f_{5}\right)$ A função $s \mapsto f(s) / s$ é crescente em $s \in(0,+\infty)$.

Se considerarmos $f(s)=\frac{m s^{3}}{m+s^{2}}, s \in \mathbb{R}$, com $m>V_{\infty}$, é possível mostrar que $f$ satisfaz as hipóteses $\left(f_{1}\right)-\left(f_{5}\right)$.

O resultado principal deste capítulo é o seguinte teorema.

Teorema 12 Sob as hipóteses $\left(V_{1}\right)-\left(V_{4}\right)$ e $\left(f_{1}\right)-\left(f_{5}\right)$, a equação $(P)$ possui uma solução fraca não trivial $u \in H^{1}\left(\mathbb{R}^{N}\right)$.

\subsection{Estrutura variacional do problema}

Seja $E:=H^{1}\left(\mathbb{R}^{N}\right)$. O funcional energia $I: E \rightarrow \mathbb{R}$ associado à equação $(P)$ é dado por

$$
I(u)=\frac{1}{2} \int_{\mathbb{R}^{N}}\left(|\nabla u|^{2}+V(x) u^{2}\right) d x-\int_{\mathbb{R}^{N}} F(u) d x
$$

com $u \in E$. É bem conhecido que, pelas hipóteses $\left(V_{2}\right)$ e $\left(V_{3}\right)$, o problema de autovalor

$$
-\Delta u+V(x) u=\lambda u, \quad u \in L^{2}\left(\mathbb{R}^{N}\right)
$$

possui uma sequência de autovalores tais que $\lambda_{1}<\lambda_{2} \leq \cdots \leq \lambda_{k}<0$ (see [17], Teorema 30, p. 150). Denotamos por $\phi_{i}$ a autofunção correspondendo ao autovalor $\lambda_{i}$, para $i \in\{1,2, \cdots, k\}$, em $H^{1}\left(\mathbb{R}^{N}\right)$. Considerando

$$
E^{-}:=\operatorname{span}\left\{\phi_{i}, i=1,2, \cdots, k\right\} \quad \text { e } \quad E^{+}:=\left(E^{-}\right)^{\perp},
$$

vemos que $E=E^{+} \oplus E^{-}$. De acordo com o Teorema 8, o espectro essencial de $-\Delta+V$ é o intervalo $\left[V_{\infty},+\infty\right)$, e isto implica que $\operatorname{dim} E^{-}<\infty$. Tendo feitas essas considerações, toda função $u \in E$ pode ser escrita como $u=u^{+}+u^{-}$unicamente, onde $u^{+} \in E^{+} \mathrm{e}$ $u^{-} \in E^{-}$. Assim, usando os argumentos do Lema 1.2 de [9], podemos introduzir um 
novo produto interno $\langle\cdot, \cdot\rangle$ em $E$, a saber, a forma bilinear definida por

$$
\langle u, v\rangle=\left\{\begin{array}{cl}
\int_{\mathbb{R}^{N}}(\nabla u \cdot \nabla v+V(x) u v) d x & \text { se } u, v \in E^{+}, \\
-\int_{\mathbb{R}^{N}}(\nabla u \cdot \nabla v+V(x) u v) d x & \text { se } u, v \in E^{-} \\
0 & \text { se } u \in E^{+} \text {e } v \in E^{-},
\end{array}\right.
$$

tal que a norma correspondente $\|\cdot\|$ é equivalente a $\|\cdot\|_{E}$, a norma usual em $E=H^{1}\left(\mathbb{R}^{N}\right)$. Além disso, o funcional $I$ pode ser escrito como

$$
I(u)=\frac{1}{2}\left\|u^{+}\right\|^{2}-\frac{1}{2}\left\|u^{-}\right\|^{2}-\int_{\mathbb{R}^{N}} F(u) d x
$$

para toda função $u=u^{+}+u^{-} \in E$. Chamamos a atenção ao fato de que, como $\lambda_{i} \neq 0$ para todo $i \in\{1,2, \cdots, k\}$, seque de (2.1) e da definição de $\phi_{i}$ que

$$
\int_{\mathbb{R}^{N}} u^{+}(x) v^{-}(x) d x=0
$$

para toda função $u^{+} \in E^{+}$e $v^{-} \in E^{-}$.

Lembramos que, dado $(X,\|\cdot\|)$ um espaço de Banach, dizemos que um funcional $I \in C^{1}(X, \mathbb{R})$ satisfaz a condição de Cerami, $(C)$, se toda sequência $\left(z_{n}\right) \subset X$ com $\left|I\left(z_{n}\right)\right|<M$, para alguma constante $M>0$, e $\left\|I^{\prime}\left(z_{n}\right)\right\|_{E^{*}}\left(1+\left\|z_{n}\right\|\right) \rightarrow 0$ possui uma subsequência $z_{n_{k}} \rightarrow z$ em $X$.

\subsection{Limitação das sequências de Cerami}

Uma consequência imediata das hipóteses $\left(f_{1}\right)$ e $\left(f_{2}\right)$ é o seguinte lema.

Lema 13 Suponha que $\left(f_{1}\right)$ e $\left(f_{2}\right)$ sejam verdadeiras. Então, dados $\varepsilon>0$ e $2 \leq p \leq 2^{*}$, existe $C(\varepsilon, p)=C_{\varepsilon}>0$ tal que

$$
|f(s)| \leq \varepsilon|s|+C_{\varepsilon}|s|^{p-1} \quad \text { e }|F(s)| \leq \varepsilon|s|^{2}+C_{\varepsilon}|s|^{p}
$$

para todo $s \in \mathbb{R}$.

Demonstração. Pelas hipóteses $\left(f_{1}\right)$ e $\left(f_{2}\right)$, dado $\varepsilon>0$, existem $R, \delta>0$ tais que $R>\delta$ com

$$
|f(t)| \leq \varepsilon|t| \text { sempre que }|t|<\delta
$$


$\mathrm{e}$

$$
|f(t)| \leq \varepsilon|t|+m|t| \text { sempre que }|t|>R
$$

Para os valores de $t$ tais que $|t|>R$, vale $|t|<\frac{|t|^{p-1}}{R^{p-2}}$. Portanto, (2.4) se torna

$$
|f(t)| \leq \varepsilon|t|+\frac{m}{R^{p-2}}|t|^{p-1} \text { sempre que }|t|>R .
$$

Pela hipótese $\left(f_{2}\right)$, temos

$$
|f(t)| \leq m|t| \text { sempre que } \delta \leq|t| \leq R
$$

Logo, para valores de $t$ em que $\delta \leq|t| \leq R$, obtemos

$$
|f(t)| \leq \frac{m}{\delta^{p-2}}|t|^{p-1} \text { sempre que } \delta \leq|t| \leq R
$$

Segue de (2.3), (2.5) e (2.6) que

$$
|f(t)| \leq \varepsilon|t|+\left(\frac{m}{R^{p-2}}+\frac{m}{\delta^{p-2}}\right)|t|^{p-1} \text { para todo } t \in \mathbb{R} .
$$

Chamando $C_{\varepsilon}:=\left(\frac{m}{R^{p-2}}+\frac{m}{\delta^{p-2}}\right)$ e, em seguida, integrando a desigualdade $(2.7)$ de 0 a $s$, obtemos

$$
|F(s)| \leq \frac{\varepsilon}{2}|s|^{2}+\frac{C_{\varepsilon}}{p}|s|^{p} \text { para todo } s \in \mathbb{R}
$$

concluindo a demonstração do lema.

Antes de enunciarmos o próximo resultado, observamos que, se $\left(v_{n}\right)$ é uma sequência limitada em $E$, então $\left(v_{n}\right)$ satisfaz apenas uma das condições a seguir.

(i) vanishing: para todo $r>0, \limsup _{n \rightarrow \infty} \sup _{y \in \mathbb{R}^{N}} \int_{B(y, r)}\left|v_{n}\right|^{2} d x=0$;

(ii) nonvanishing: existem $r, \eta>0$ e uma sequência $\left(y_{n}\right) \subset \mathbb{R}^{N}$ tais que

$$
\limsup _{n \rightarrow \infty} \int_{B\left(y_{n}, r\right)}\left|v_{n}\right|^{2} d x>\eta .
$$

Lema 14 Seja $\left(u_{n}\right) \subset$ E uma sequência tal que $I\left(u_{n}\right) \rightarrow c>0$ e $\left\|I^{\prime}\left(u_{n}\right)\right\|_{E^{*}}\left(1+\left\|u_{n}\right\|\right) \rightarrow$ 0 quando $n \rightarrow+\infty$. Então, $\left(u_{n}\right)$ possui uma subsequência limitada. 
Demonstração. Vamos supor que $\left\|u_{n}\right\| \rightarrow \infty$ e obter uma contradição. Para isso, considere $v_{n}=\frac{u_{n}}{\left\|u_{n}\right\|}$ e observe que $\left\|v_{n}\right\|=1$. Embora a sequência $\left(v_{n}\right)$ seja limitada, vamos mostrar que não vale $(i)$ nem $(i i)$, concluindo com isso um absurdo. Primeiramente, suponha que valha $(i i)$ para a sequência $\left(v_{n}\right)$. Escreva $f(s)=m s+(f(s)-m s)=m s+f_{\infty}(s)$ e considere $\varphi \in C_{0}^{\infty}\left(\mathbb{R}^{N}\right)$. Pela equivalência das normas $\|\cdot\| \mathrm{e}\|\cdot\|_{E}$, existem constantes $C_{1}, C_{2}>0$ tais que

$$
\|w\| \leq C_{1}\|w\|_{H^{1}\left(\mathbb{R}^{N}\right)} \leq C_{2}\|w\| \text {, para toda função } w \in E .
$$

Seja $\left(y_{n}\right) \subset \mathbb{R}^{N}$ a sequência dada pela hipótese $(i i)$. Como a sequência $\left(u_{n}\right)$ é de Cerami e considerando $\varphi_{n}(x)=\varphi\left(x-y_{n}\right)$, temos de $(2.8)$ que

$$
\left|I^{\prime}\left(u_{n}\right) \varphi_{n}\right| \leq\left\|I^{\prime}\left(u_{n}\right)\right\|_{E^{*}}\left\|\varphi_{n}\right\| \leq C_{1}\left\|I^{\prime}\left(u_{n}\right)\right\|_{E^{*}}\left\|\varphi_{n}\right\|_{E}=C_{1}\left\|I^{\prime}\left(u_{n}\right)\right\|_{E^{*}}\|\varphi\|_{E} \rightarrow 0
$$

Logo,

$$
\begin{aligned}
o_{n}(1) & =\frac{1}{\left\|u_{n}\right\|} I^{\prime}\left(u_{n}\right) \varphi_{n}=\left\langle v_{n}^{+}-v_{n}^{-}, \varphi_{n}\right\rangle-\int_{\mathbb{R}^{N}} \frac{f\left(u_{n}\right)}{\left\|u_{n}\right\|} \varphi_{n} d x \\
& =\left\langle v_{n}^{+}-v_{n}^{-}, \varphi_{n}\right\rangle-\int_{\mathbb{R}^{N}} m v_{n} \varphi_{n} d x-\int_{\mathbb{R}^{N}} \frac{f_{\infty}\left(u_{n}\right)}{\left\|u_{n}\right\|} \varphi_{n} d x \\
& =\left\langle v_{n}^{+}-v_{n}^{-}, \varphi_{n}\right\rangle-\int_{\mathbb{R}^{N}} m v_{n} \varphi_{n} d x-\int_{\mathbb{R}^{N}} \frac{f_{\infty}\left(u_{n}\right)}{u_{n}} v_{n} \varphi_{n} d x .
\end{aligned}
$$

Considere $\tilde{v}_{n}(x)=v_{n}\left(x+y_{n}\right)$ e $\tilde{u}_{n}(x)=u_{n}\left(x+y_{n}\right)$. Observe que $\left(\tilde{v}_{n}\right)$ é limitada em $E$. De fato, de (2.8), segue que

$$
\left\|\tilde{v}_{n}\right\| \leq C_{1}\left\|\tilde{v}_{n}\right\|_{H^{1}\left(\mathbb{R}^{N}\right)}=C_{1}\left\|v_{n}\right\|_{H^{1}\left(\mathbb{R}^{N}\right)} \leq C_{2}\left\|v_{n}\right\|=C_{2}
$$

Logo, a menos de subsequência,

$$
\begin{array}{lc}
\tilde{v}_{n} \rightarrow \tilde{v} & \text { em } E, \\
\tilde{v}_{n} \rightarrow \tilde{v} & \text { em } L_{l o c}^{2}\left(\mathbb{R}^{N}\right) .
\end{array}
$$

Seja $K=\operatorname{supp}(\varphi)$. Pela hipótese $\left(f_{2}\right), \frac{|f(\cdot)|}{|\cdot|}$ é uma função limitada em $\mathbb{R} \backslash\{0\}$ com $\frac{|f(\cdot)|}{|\cdot|} \leq m$. Como vale (2.10), existe uma função $h \in L^{1}(K)$ tal que $\left|\tilde{v}_{n}(x)\right| \leq h(x)$ q.t.p. em $K$. Assim, lembrando que $f_{\infty}(s)=f(s)-m s$, temos

$$
\left|\frac{f_{\infty}\left(\tilde{u}_{n}\right)}{\tilde{u}_{n}} \tilde{v}_{n} \varphi\right| \leq 2 m h(x) \varphi \in L^{1}(K) .
$$


Observamos que $\tilde{v} \neq 0$, pois, por $(i i)$ e por $(2.10)$,

$$
\int_{B(0, r)} \tilde{v}^{2} d x=\limsup _{n \rightarrow \infty} \int_{B(0, r)} \tilde{v}_{n}^{2} d x=\limsup _{n \rightarrow \infty} \int_{B\left(y_{n}, r\right)} v_{n}^{2} d x>\eta>0 .
$$

Pela hipótese $\left(f_{1}\right)$, temos que $\lim _{|s| \rightarrow \infty} \frac{f_{\infty}(s)}{s}=0$. Assim, por $(2.8),\left\|\tilde{u}_{n}\right\| \rightarrow \infty$ e, portanto, de (2.11) e do Teorema da Convergência Dominada de Lebesgue, segue que

$$
\int_{\mathbb{R}^{N}} \frac{f_{\infty}\left(u_{n}\right)}{u_{n}} v_{n} \varphi_{n} d x=\int_{K} \frac{f_{\infty}\left(\tilde{u}_{n}\right)}{\tilde{u}_{n}} \tilde{v}_{n} \varphi d x=\int_{K} \frac{f_{\infty}\left(\tilde{v}_{n}\left\|\tilde{u}_{n}\right\|\right)}{\tilde{v}_{n}\left\|\tilde{u}_{n}\right\|} \tilde{v}_{n} \varphi d x \rightarrow 0 .
$$

Assim, de (2.9), (2.10), (2.12) o do Teorema da Mudança de Variável, tem-se

$$
\begin{aligned}
o_{n}(1)= & \frac{1}{\left\|u_{n}\right\|} I^{\prime}\left(u_{n}\right) \varphi_{n} \\
= & \left\langle v_{n}^{+}-v_{n}^{-}, \varphi_{n}\right\rangle-\int_{\mathbb{R}^{N}} m v_{n} \varphi_{n} d x-\int_{\mathbb{R}^{N}} \frac{f_{\infty}\left(u_{n}\right)}{\left\|u_{n}\right\|} \varphi_{n} d x \\
= & \int_{\mathbb{R}^{N}}\left(\nabla v_{n}^{+} \nabla \varphi\left(x-y_{n}\right)+V(x) v_{n}^{+} \varphi\left(x-y_{n}\right)\right) d x+\int_{\mathbb{R}^{N}} \nabla v_{n}^{-} \nabla \varphi\left(x-y_{n}\right) d x \\
& +\int_{\mathbb{R}^{N}} V(x) v_{n}^{-} \varphi\left(x-y_{n}\right) d x-\int_{\mathbb{R}^{N}} m v_{n} \varphi_{n} d x-o_{n}(1) \\
= & \int_{\mathbb{R}^{N}}\left(\nabla \tilde{v}_{n}^{+} \nabla \varphi+V\left(x+y_{n}\right) \tilde{v}_{n}^{+} \varphi\right) d x+\int_{\mathbb{R}^{N}}\left(\nabla \tilde{v}_{n}^{-} \nabla \varphi+V\left(x+y_{n}\right) \tilde{v}_{n}^{-} \varphi\right) d x \\
& -\int_{\mathbb{R}^{N}} m \tilde{v}_{n} \varphi d x .
\end{aligned}
$$

Caso 1: $\left|y_{n}\right| \rightarrow \infty$. Neste caso, a hipótese $\left(V_{2}\right)$ garante que $V\left(x+y_{n}\right)$ converge para $V_{\infty}$ q.t.p. em $\mathbb{R}^{N}$ quando $n \rightarrow \infty$. Segue de (2.9) que

$$
\begin{aligned}
o_{n}(1)= & \int_{K}\left(\nabla \tilde{v}_{n}^{+} \nabla \varphi+\left(V_{\infty}+o_{n}(1)\right) \tilde{v}_{n}^{+} \varphi\right) d x+\int_{K}\left(\nabla \tilde{v}_{n}^{-} \nabla \varphi+\left(V_{\infty}+o_{n}(1)\right) \tilde{v}_{n}^{-} \varphi\right) d x \\
& -\int_{K} m \tilde{v}_{n} \varphi d x .
\end{aligned}
$$

Fazendo $n \rightarrow \infty$ em (2.14) e lembrando que vale (2.10), para toda função $\varphi \in C_{0}^{\infty}\left(\mathbb{R}^{N}\right)$, obtemos

$$
\int_{\mathbb{R}^{N}}\left(\nabla\left(\tilde{v}^{+}-\tilde{v}^{-}\right) \nabla \varphi+V_{\infty}\left(\tilde{v}^{+}-\tilde{v}^{-}\right) \varphi\right) d x-\int_{\mathbb{R}^{N}} m \tilde{v} \varphi d x=0
$$

isto é, $\tilde{v} \neq 0$ é solução fraca do problema

$$
-\Delta \tilde{v}+V_{\infty} \tilde{v}=m \tilde{v} \text { em } \mathbb{R}^{N}
$$

Uma vez que $V_{\infty}<m$ e não existe autofunção do laplaciano em $\mathbb{R}^{N}$, isso é um absurdo. Logo, (ii) não pode valer quando $\left|y_{n}\right| \rightarrow \infty$. 
Caso 2: $\left(y_{n}\right)$ é uma sequência limitada. Neste caso, como por (2.8), $\left\|\tilde{u}_{n}\right\| \rightarrow \infty$, segue de $(2.10)$ que

$$
0 \neq|\tilde{v}(x)|=\lim _{n \rightarrow \infty}\left|\tilde{v}_{n}(x)\right|=\lim _{n \rightarrow \infty} \frac{\left|\tilde{u}_{n}(x)\right|}{\left\|\tilde{u}_{n}\right\|}
$$

q.t.p. em $\Omega$ para algum $\Omega \subset B(0, r) \operatorname{com} \mu(\Omega)>0$. Visto que $\left\|\tilde{u}_{n}\right\| \rightarrow \infty$, temos $\tilde{u}_{n}(x) \rightarrow \infty$ q.t.p. em $\Omega$. Logo, a hipótese $\left(f_{3}\right)$ e o Lema de Fatou nos fornecem

$$
\begin{aligned}
\liminf _{n \rightarrow \infty} \int_{\mathbb{R}^{N}}\left(\frac{1}{2} f\left(u_{n}\right) u_{n}-F\left(u_{n}\right)\right) d x & =\liminf _{n \rightarrow \infty} \int_{\mathbb{R}^{N}}\left(\frac{1}{2} f\left(\tilde{u}_{n}\right) \tilde{u}_{n}-F\left(\tilde{u}_{n}\right)\right) d x \\
& \geq \liminf _{n \rightarrow \infty} \int_{\Omega}\left(\frac{1}{2} f\left(\tilde{u}_{n}\right) \tilde{u}_{n}-F\left(\tilde{u}_{n}\right)\right) d x \\
& \geq \int_{\Omega} \liminf _{n \rightarrow \infty}\left(\frac{1}{2} f\left(\tilde{u}_{n}\right) \tilde{u}_{n}-F\left(\tilde{u}_{n}\right)\right) d x \\
& =+\infty
\end{aligned}
$$

Todavia, isso contradiz o fato de

$$
\int_{\mathbb{R}^{N}}\left(\frac{1}{2} f\left(u_{n}\right) u_{n}-F\left(u_{n}\right)\right) d x=I\left(u_{n}\right)-\frac{1}{2} I^{\prime}\left(u_{n}\right) u_{n}=c+o_{n}(1)
$$

Logo, o Caso 2 também não vale. Isso mostra que não pode ocorrer a hipótese (ii) para a sequência $\left(v_{n}\right)$. Suponha, agora, que valha a hipótese $(i)$ para a sequência $\left(v_{n}\right)$ e vamos encontrar novamente uma contradição. Ora, como a sequência $\left(u_{n}\right)$ é de Cerami, $\left\|I^{\prime}\left(u_{n}\right)\right\|_{E^{*}}^{2}\left\|u_{n}\right\|^{2} \rightarrow 0$ quando $n \rightarrow \infty$. Ou seja, $\left\|I^{\prime}\left(u_{n}\right)\right\|_{E^{*}}^{2}\left\|u_{n}^{+}\right\|^{2}+\left\|I^{\prime}\left(u_{n}\right)\right\|_{E^{*}}^{2}\left\|u_{n}^{-}\right\|^{2} \rightarrow 0$. Assim, tanto $I^{\prime}\left(u_{n}\right) u_{n}^{-} \rightarrow 0$ quanto $I^{\prime}\left(u_{n}\right) u_{n}^{+} \rightarrow 0$. Logo,

$$
o_{n}(1)=I^{\prime}\left(u_{n}\right) \frac{u_{n}^{+}}{\left\|u_{n}\right\|^{2}}=\frac{1}{\left\|u_{n}\right\|} I^{\prime}\left(u_{n}\right) v_{n}^{+}=\left\|v_{n}^{+}\right\|^{2}-\int_{\mathbb{R}^{N}}\left(\frac{f\left(u_{n}\right)}{u_{n}} v_{n} v_{n}^{+}\right) d x
$$

e

$$
o_{n}(1)=I^{\prime}\left(u_{n}\right) \frac{u_{n}^{-}}{\left\|u_{n}\right\|^{2}}=\frac{1}{\left\|u_{n}\right\|} I^{\prime}\left(u_{n}\right) v_{n}^{-}=-\left\|v_{n}^{-}\right\|^{2}-\int_{\mathbb{R}^{N}}\left(\frac{f\left(u_{n}\right)}{u_{n}} v_{n} v_{n}^{-}\right) d x
$$

Subtraindo de (2.15) a equação (2.16), temos

$$
\begin{aligned}
o_{n}(1) & =\left\|v_{n}^{+}\right\|^{2}+\left\|v_{n}^{-}\right\|^{2}-\int_{\mathbb{R}^{N}}\left(\frac{f\left(u_{n}\right)}{u_{n}} v_{n}\left(v_{n}^{+}-v_{n}^{-}\right)\right) d x \\
& =\left\|v_{n}\right\|^{2}-\int_{\mathbb{R}^{N}}\left(\frac{f\left(u_{n}\right)}{u_{n}} v_{n}\left(v_{n}^{+}-v_{n}^{-}\right)\right) d x \\
& =1-\int_{\mathbb{R}^{N}}\left(\frac{f\left(u_{n}\right)}{u_{n}} v_{n}\left(v_{n}^{+}-v_{n}^{-}\right)\right) d x .
\end{aligned}
$$


Então, necessariamente, quando $n \rightarrow \infty$,

$$
\int_{\mathbb{R}^{N}}\left(\frac{f\left(u_{n}\right)}{u_{n}} v_{n}\left(v_{n}^{+}-v_{n}^{-}\right)\right) d x \rightarrow 1
$$

Como $E$ está imerso continuamente em $L^{2}\left(\mathbb{R}^{N}\right)$, seja $\mu_{0}>0$ tal que

$$
\|w\|^{2} \geq \mu_{0}\|w\|_{L^{2}\left(\mathbb{R}^{N}\right)}^{2}
$$

para todo $w \in E$. Dado $0<\varepsilon<\frac{1}{2} \mu_{0}$, pela hipótese $\left(f_{1}\right)$, existe $\delta>0$ tal que

$$
\frac{|f(s)|}{|s|} \leq \varepsilon \text { sempre que } 0 \neq|s|<\delta
$$

Para cada $n \in \mathbb{N}$, considere o conjunto

$$
\tilde{\Omega}_{n}=\left\{x \in \mathbb{R}^{N} ;\left|u_{n}(x)\right|<\delta\right\}
$$

Então, da escolha de $\varepsilon$ e da desigualdade de Hölder, segue que

$$
\begin{aligned}
\int_{\tilde{\Omega}_{n}}\left(\frac{f\left(u_{n}\right)}{u_{n}} v_{n}\left(v_{n}^{+}-v_{n}^{-}\right)\right) d x & \leq \varepsilon \int_{\tilde{\Omega}_{n}}\left|v_{n} \| v_{n}^{+}-v_{n}^{-}\right| d x \\
& \leq \varepsilon\left(\left\|v_{n}\right\|_{L^{2}\left(\mathbb{R}^{N}\right)}\left\|v_{n}^{+}\right\|_{L^{2}\left(\mathbb{R}^{N}\right)}+\left\|v_{n}\right\|_{L^{2}\left(\mathbb{R}^{N}\right)}\left\|v_{n}^{-}\right\|_{L^{2}\left(\mathbb{R}^{N}\right)}\right) \\
& \left.\leq 2 \varepsilon\left\|v_{n}\right\|_{L^{2}\left(\mathbb{R}^{N}\right.}^{2}\right) \\
& \leq \frac{2 \varepsilon}{\mu_{0}}\left\|v_{n}\right\|^{2}=\frac{2 \varepsilon}{\mu_{0}}<1,
\end{aligned}
$$

A partir de (2.17), concluímos que

$$
\liminf _{n \rightarrow \infty} \int_{\mathbb{R}^{N} \backslash \tilde{\Omega}_{n}}\left(\frac{f\left(u_{n}\right)}{u_{n}} v_{n}\left(v_{n}^{+}-v_{n}^{-}\right)\right) d x>0 .
$$

Do fato de $\frac{|f(\cdot)|}{|\cdot|}$ ser limitada e novamente pela desigualdade de Hölder com expoente $\frac{p}{2}>1$, vale que

$$
\int_{\mathbb{R}^{N} \backslash \tilde{\Omega}_{n}}\left(\frac{f\left(u_{n}\right)}{u_{n}} v_{n}\left(v_{n}^{+}-v_{n}^{-}\right)\right) d x \leq C \mu\left(\mathbb{R}^{N} \backslash \tilde{\Omega}_{n}\right)^{\frac{p-2}{p}}\left\|v_{n}\right\|_{L^{p}\left(\mathbb{R}^{N}\right)}^{\frac{2}{p}}
$$

Desde que vale $(i)$, o Lema de Lions garante que $\left\|v_{n}\right\|_{L^{p}\left(\mathbb{R}^{N}\right)} \rightarrow 0$, pois $2<p<2^{*}$. Portanto, a menos de subsequência, segue de (2.18) que

$$
\mu\left(\mathbb{R}^{N} \backslash \tilde{\Omega}_{n}\right) \rightarrow \infty \quad \text { quando } \quad n \rightarrow \infty
$$


Pela hipótese $\left(f_{3}\right)$, existe $R>0$ tal que, se $|s|>R$,

$$
\frac{1}{2} f(s) s-F(s)>1
$$

Sem perda de generalidade, podemos supor $0<\delta<R$. Para cada $n \in \mathbb{N}$, considere $A_{n}:=\left\{x \in \mathbb{R}^{N} ;\left|u_{n}(x)\right|>R\right\}$. Logo,

$$
\begin{aligned}
c+o_{n}(1) & =I\left(u_{n}\right)-\frac{1}{2} I^{\prime}\left(u_{n}\right) u_{n} \\
& =\int_{\mathbb{R}^{N}}\left(\frac{1}{2} f\left(u_{n}\right) u_{n}-F\left(u_{n}\right)\right) d x \\
& \geq \int_{A_{n}}\left(\frac{1}{2} f\left(u_{n}\right) u_{n}-F\left(u_{n}\right)\right) d x>\mu\left(A_{n}\right)
\end{aligned}
$$

o que implica que a sequência $\left(\mu\left(A_{n}\right)\right)$ é limitada. Considere também $B_{n}:=\{x \in$ $\left.\mathbb{R}^{N} ; \delta \leq\left|u_{n}(x)\right| \leq R\right\}$. Como $\mathbb{R}^{N} \backslash \tilde{\Omega}_{n}=A_{n} \cup B_{n}$, temos

$$
\mu\left(\mathbb{R}^{N} \backslash \tilde{\Omega}_{n}\right)=\mu\left(A_{n}\right)+\mu\left(B_{n}\right)
$$

Segue de $(2.20)$ e da limitação da sequência $\left(\mu\left(A_{n}\right)\right)$ que

$$
\mu\left(B_{n}\right) \rightarrow \infty
$$

Pela hipótese $\left(f_{3}\right)$, sendo o intervalo $[\delta, R]$ compacto e as funções $f$ e $F$ contínuas, temos que $\bar{\delta}:=\inf _{s \in[\delta, R]}\left(\frac{1}{2} f(s) s-F(s)\right)>0$. Assim, de (2.21),

$$
\begin{aligned}
\int_{\mathbb{R}^{N}}\left(\frac{1}{2} f\left(u_{n}\right) u_{n}-F\left(u_{n}\right)\right) d x & \geq \int_{B_{n}}\left(\frac{1}{2} f\left(u_{n}\right) u_{n}-F\left(u_{n}\right)\right) d x \\
& \geq \bar{\delta} \mu\left(B_{n}\right) \rightarrow \infty
\end{aligned}
$$

Isso novamente contradiz o fato de

$$
\int_{\mathbb{R}^{N}}\left(\frac{1}{2} f\left(u_{n}\right) u_{n}-F\left(u_{n}\right)\right) d x=I\left(u_{n}\right)-\frac{1}{2} I^{\prime}\left(u_{n}\right) u_{n}=c+o_{n}(1) .
$$

Logo, também não pode ocorrer $(i)$ para a sequência $\left(v_{n}\right)$. Mas isso é um absurdo. Concluímos que, a menos de subsequência, $\left(u_{n}\right)$ é limitada. 


\subsection{Uma solução não trivial}

No que segue, provaremos que o funcional $I$ satisfaz a geometria do Teorema de Linking, provado em [29], Proposição 2.10, e demonstraremos nosso resultado principal.

Teorema 15 (Teorema de Linking para sequências de Cerami) Seja $E=E^{+} \oplus E^{-} u m$ espaço de Banach com dim $E^{-}<\infty$. Sejam $R>\rho>0$ e $u \in E^{+}$um elemento fixo tal que $\|u\|=\rho$. Considere

$$
\begin{aligned}
M & :=\left\{w=t u+v^{-}:\|w\| \leq R, t \geq 0, v^{-} \in E^{-}\right\} \\
M_{0} & :=\left\{w=t u+v^{-}: v^{-} \in E^{-},\|w\|=R, t \geq 0 \text { ou }\|w\| \leq R, t=0\right\} \\
N_{\rho} & :=\left\{w \in E^{+}:\|w\|=\rho\right\} .
\end{aligned}
$$

Seja $I \in C^{1}(E, \mathbb{R})$ tal que

$$
b:=\inf _{N_{\rho}} I>a:=\max _{M_{0}} I .
$$

Então, $c \geq b$ e existe uma sequência de Cerami no nível c para o funcional I, onde

$$
c:=\inf _{\gamma \in \Gamma} \max _{w \in M} I(\gamma(w)), \quad \Gamma:=\left\{\gamma \in C(M, E):\left.\gamma\right|_{M_{0}}=I d\right\}
$$

Considere o seguinte problema limite

$$
-\Delta w+V_{\infty} w=f(w) \text { em } \mathbb{R}^{N}
$$

e o funcional energia associado à equação $\left(P_{\infty}\right)$ dado por

$$
I_{\infty}(w)=\frac{1}{2} \int_{\mathbb{R}^{N}}\left(|\nabla w|^{2}+V_{\infty} w^{2}\right) d x-\int_{\mathbb{R}^{N}} F(w) d x
$$

$\operatorname{com} w \in E$. Seja $u_{0} \in E$ uma solução radial, contínua, positiva e de energia mínima da equação $\left(P_{\infty}\right)$ tal que $I_{\infty}\left(u_{0}\right)=c_{\infty}>0$. Desde que $V_{\infty}<m$, a existência de tal solução é verdadeira por [4].

Para simplificar a notação, dados $w \in E$ e $y \in \mathbb{R}^{N}$, escreveremos $w^{+}(\cdot-y)\left(\right.$ ou $\left.w^{-}(\cdot-y)\right)$ nos referindo à projeção em $E^{+}$(respectivamente, em $E^{-}$) da função transladada $w(\cdot-y)$.

Lema 16 Sejam $w$ e $v$ funções em $L^{2}\left(\mathbb{R}^{N}\right)$. Então,

$$
\int_{\mathbb{R}^{N}} w(x-y) v(x) d x \rightarrow 0 \quad \text { quando } \quad|y| \rightarrow \infty .
$$


Demonstração. Como $w, v \in L^{2}\left(\mathbb{R}^{N}\right)$, dado $\varepsilon>0$, existe $R>0$ tal que

$$
\int_{B_{R}^{c}(0)} w^{2}(x) d x<\varepsilon \quad \text { e } \quad \int_{B_{R}^{c}(0)} v^{2}(x) d x<\varepsilon .
$$

De (2.22) e da desigualdade de Hölder,

$$
\begin{aligned}
\left|\int_{B_{R}^{c}(0)} w(x-y) v(x) d x\right| & \leq\left(\int_{B_{R}^{c}(0)} w^{2}(x-y) d x\right)^{\frac{1}{2}}\left(\int_{B_{R}^{c}(0)} v^{2}(x) d x\right)^{\frac{1}{2}} \\
& \leq\left(\int_{\mathbb{R}^{N}} w^{2}(z) d z\right)^{\frac{1}{2}}\left(\int_{B_{R}^{c}(0)} v^{2}(x) d x\right)^{\frac{1}{2}} \\
& <\varepsilon^{\frac{1}{2}}\|w\|_{L^{2}\left(\mathbb{R}^{N}\right)}
\end{aligned}
$$

Escolhendo agora $y \in \mathbb{R}^{N}$ com $|y|$ suficientemente grande de tal forma que $B_{R}(y) \cap$ $B_{R}(0)=\emptyset$, isto é, de tal forma que $B_{R}(y) \subset B_{R}^{c}(0)$, obtemos de $(2.22)$

$$
\begin{aligned}
\left|\int_{B_{R}(0)} w(x-y) v(x) d x\right| & \leq\left(\int_{B_{R}(0)} w^{2}(x-y) d x\right)^{\frac{1}{2}}\left(\int_{B_{R}(0)} v^{2}(x) d x\right)^{\frac{1}{2}} \\
& \leq\left(\int_{B_{R}(y)} w^{2}(z) d z\right)^{\frac{1}{2}}\left(\int_{B_{R}(0)} v^{2}(x) d x\right)^{\frac{1}{2}} \\
& \leq\left(\int_{B_{R}^{c}(0)} w^{2}(z) d z\right)^{\frac{1}{2}}\left(\int_{\mathbb{R}^{N}} v^{2}(x) d x\right)^{\frac{1}{2}} \\
& <\varepsilon^{\frac{1}{2}}\|v\|_{L^{2}\left(\mathbb{R}^{N}\right)}
\end{aligned}
$$

Assim, de (2.23) e (2.24), segue que

$$
\begin{aligned}
\left|\int_{\mathbb{R}^{N}} w(x-y) v(x) d x\right| & \leq\left|\int_{B_{R}(0)} w(x-y) v(x) d x\right|+\left|\int_{B_{R}^{c}(0)} w(x-y) v(x) d x\right| \\
& <\varepsilon^{\frac{1}{2}\left(\|v\|_{L^{2}\left(\mathbb{R}^{N}\right)}+\|w\|_{L^{2}\left(\mathbb{R}^{N}\right)}\right)}
\end{aligned}
$$

para $|y|$ suficientemente grande. Como $\varepsilon>0$ foi tomado arbitrariamente, o lema está provado.

Para $R>0$ e $y \in \mathbb{R}^{N}$, considere

$$
M=\left\{w=t u_{0}^{+}(\cdot-y)+v^{-} ;\|w\| \leq R, t \geq 0, v^{-} \in E^{-}\right\}
$$

$\mathrm{e}$

$$
M_{0}=\left\{w=t u_{0}^{+}(\cdot-y)+v^{-} ; v^{-} \in E^{-},\|w\|=R, t \geq 0 \text { ou }\|w\| \leq R, t=0\right\}
$$


Lema 17 Existem $R>0$ e $y \in \mathbb{R}^{N}$ com $R,|y|$ suficientemente grandes tais que

$$
\left.I\right|_{M_{0}} \leq 0
$$

Demonstração. Observamos que $M_{0}=M_{1} \cup M_{2}$ é uma união disjunta, onde

$$
M_{1}=\left\{w=t u_{0}^{+}(\cdot-y)+v^{-} ; v^{-} \in E^{-},\|w\| \leq R, t=0\right\}
$$

$\mathrm{e}$

$$
M_{2}=\left\{w=t u_{0}^{+}(\cdot-y)+v^{-} ; v^{-} \in E^{-},\|w\|=R, t>0\right\} .
$$

Como $M_{1} \subset E^{-}$, temos que $I(w) \leq 0$ sempre que $w \in M_{1}$. Sejam $R>0$ e $w \in M_{2}$ com $\|w\|=R$. Escrevendo $w=\|w\| \frac{w}{\|w\|}=\|w\| u(w)=\|w\|\left(\lambda(w) u_{0}^{+}(\cdot-y)+v^{-}(w)\right)$, temos

$$
\begin{aligned}
I(\|w\| u(w)) & =\frac{1}{2}\|w\|^{2} \lambda^{2}(w)\left\|u_{0}^{+}(\cdot-y)\right\|^{2}-\frac{1}{2}\|w\|^{2}\left\|v^{-}(w)\right\|^{2}-\int_{\mathbb{R}^{N}} F(\|w\| u(w)) d x \\
& =\frac{1}{2}\|w\|^{2}\left\{\lambda^{2}(w)\left\|u_{0}^{+}(\cdot-y)\right\|^{2}-\left\|v^{-}(w)\right\|^{2}-2 \int_{\mathbb{R}^{N}} \frac{F(R u(w))}{(R u(w))^{2}} u^{2}(w) d x\right\} .
\end{aligned}
$$

Para simplificar a notação, escreveremos $\lambda, u$ e $v^{-}$ao invés de $\lambda(w), u(w)$ e $v^{-}(w)$, respectivamente. Pela hipótese $\left(f_{2}\right), \lim _{s \rightarrow \infty} \frac{F(s)}{s^{2}}=\frac{1}{2} m$ e $\frac{F(s)}{s^{2}} \leq \frac{m}{2}$ para todo $s \neq 0$. Então,

$$
\left|\frac{F(R u)}{(R u)^{2}} u^{2}\right| \leq \frac{m}{2} u^{2} \in L^{1}\left(\mathbb{R}^{N}\right)
$$

e, pelo Teorema da Convergência Dominada de Lebesgue,

$$
\lim _{R \rightarrow \infty} \int_{\mathbb{R}^{N}}\left(\frac{F(R u)}{(R u)^{2}}-\frac{m}{2}\right) u^{2} d x=0
$$

para todo $u \in E$ tal que $\|u\|=1$. Visto que o conjunto $M_{2}$ está contido em um subespaço de dimensão finita de $E, w=R u \in M_{2} \operatorname{com}\|u\|=1$ e, pela hipótese $\left(f_{1}\right), s \mapsto \frac{F(s)}{s^{2}}$ é uma função contínua, então o limite (2.25) é uniforme em $u$ (veja Apêndice A, Lema 43). Lembrando de (2.2) que

$$
\int_{\mathbb{R}^{N}} u_{0}^{+}(x-y) v^{-} d x=0
$$


temos

$$
\begin{aligned}
I(w)= & \frac{1}{2}\|w\|^{2}\left\{\lambda^{2}\left\|u_{0}^{+}(\cdot-y)\right\|^{2}-\left\|v^{-}\right\|^{2}-m \int_{\mathbb{R}^{N}} u^{2} d x+o_{R}(1)\right\} \\
= & \frac{1}{2}\|w\|^{2}\left\{\lambda^{2}\left\|u_{0}^{+}(\cdot-y)\right\|^{2}-\left\|v^{-}\right\|^{2}-m \int_{\mathbb{R}^{N}}\left(\lambda u_{0}^{+}(x-y)+v^{-}\right)^{2} d x+o_{R}(1)\right\} \\
= & \frac{1}{2}\|w\|^{2}\left\{\lambda^{2}\left\|u_{0}^{+}(\cdot-y)\right\|^{2}-\left\|v^{-}\right\|^{2}-m \lambda^{2} \int_{\mathbb{R}^{N}}\left(u_{0}^{+}\right)^{2}(x-y) d x\right. \\
& \left.-m \int_{\mathbb{R}^{N}}\left(v^{-}\right)^{2} d x+o_{R}(1)\right\} \\
\leq & \frac{1}{2}\|w\|^{2}\left\{\lambda^{2}\left[\left\|u_{0}^{+}(\cdot-y)\right\|^{2}-m \int_{\mathbb{R}^{N}}\left(u_{0}^{+}\right)^{2}(x-y) d x\right]+o_{R}(1)\right\}
\end{aligned}
$$

Pela hipótese $\left(V_{1}\right), V(x) \leq V_{\infty}$ para todo $x \in \mathbb{R}^{N}$. Segue que

$$
\begin{aligned}
\left\|u_{0}^{+}(\cdot-y)\right\|^{2} & =\int_{\mathbb{R}^{N}}\left(\left|\nabla u_{0}^{+}(x-y)\right|^{2}+V(x)\left(u_{0}^{+}\right)^{2}(x-y)\right) d x \\
& \leq \int_{\mathbb{R}^{N}}\left(\left|\nabla u_{0}^{+}(x-y)\right|^{2}+V_{\infty}\left(u_{0}^{+}\right)^{2}(x-y)\right) d x \\
& =\left\|u_{0}^{+}(\cdot-y)\right\|_{V_{\infty}}^{2} \\
& \leq\left\|u_{0}(\cdot-y)\right\|_{V_{\infty}}^{2} .
\end{aligned}
$$

Agora, como $u_{0}$ é ponto crítico do funcional $I_{\infty}$, temos pela invariância por translação de $I_{\infty}$ que $u_{0}(\cdot-y)$ também o é. Portanto, $I_{\infty}^{\prime}\left(u_{0}(\cdot-y)\right) u_{0}(\cdot-y)=0$, isto é,

$$
\left\|u_{0}(\cdot-y)\right\|_{V_{\infty}}^{2}=\int_{\mathbb{R}^{N}} f\left(u_{0}(x-y)\right) u_{0}(x-y) d x
$$

De $(2.27)$ e $(2.28)$,

$$
\left\|u_{0}^{+}(\cdot-y)\right\|^{2} \leq \int_{\mathbb{R}^{N}} f\left(u_{0}(x-y)\right) u_{0}(x-y) d x
$$

Substituindo (2.29) em (2.26) e, em seguida, somando e subtraindo a integral $m \int_{\mathbb{R}^{N}} u_{0}^{2}(x-y) d x$, obtemos

$$
\begin{aligned}
I(w) \leq & \frac{1}{2}\|w\|^{2}\left\{\lambda^{2}\left[\int_{\mathbb{R}^{N}} f\left(u_{0}(x-y)\right) u_{0}(x-y) d x-m \int_{\mathbb{R}^{N}}\left(u_{0}^{+}\right)^{2}(x-y) d x\right]+o_{R}(1)\right\} \\
= & \frac{1}{2}\|w\|^{2}\left\{\lambda ^ { 2 } \left[\int_{\mathbb{R}^{N}} f\left(u_{0}(x-y)\right) u_{0}(x-y) d x-m \int_{\mathbb{R}^{N}} u_{0}^{2}(x-y) d x\right.\right. \\
& \left.\left.+m \int_{\mathbb{R}^{N}}\left[u_{0}^{2}(x-y)-\left(u_{0}^{+}\right)^{2}(x-y)\right] d x\right]+o_{R}(1)\right\}
\end{aligned}
$$




$$
\begin{aligned}
= & \frac{1}{2}\|w\|^{2}\left\{\lambda ^ { 2 } \left[\int_{\mathbb{R}^{N}} f\left(u_{0}(z)\right) u_{0}(z) d z-m \int_{\mathbb{R}^{N}} u_{0}^{2}(z) d z\right.\right. \\
& \left.\left.+m \int_{\mathbb{R}^{N}}\left[u_{0}^{2}(x-y)-\left(u_{0}^{+}\right)^{2}(x-y)\right] d x\right]+o_{R}(1)\right\} .
\end{aligned}
$$

Vamos agora estimar as integrais

$$
\int_{\mathbb{R}^{N}} f\left(u_{0}(z)\right) u_{0}(z) d z-m \int_{\mathbb{R}^{N}} u_{0}^{2}(z) d z \quad \text { e } \quad \int_{\mathbb{R}^{N}}\left[u_{0}^{2}(x-y)-\left(u_{0}^{+}\right)^{2}(x-y)\right] d x
$$

Ora, sendo $u_{0}$ radial e contínua, a função $\frac{f\left(u_{0}(\cdot)\right)}{u_{0}(\cdot)}$ assume seu valor máximo em, digamos, $x_{0} \in \mathbb{R}^{N}$. Assim, já que, pela hipótese $\left(f_{2}\right), \frac{f(s)}{s}<m$ para todo $s \neq 0$,

$$
\begin{aligned}
\int_{\mathbb{R}^{N}} f\left(u_{0}(z)\right) u_{0}(z) d z-m \int_{\mathbb{R}^{N}} u_{0}^{2}(z) d z & =\int_{\mathbb{R}^{N}}\left(\frac{f\left(u_{0}(z)\right)}{u_{0}(z)}-m\right) u_{0}^{2}(z) d z \\
& \leq\left(\frac{f\left(u_{0}\left(x_{0}\right)\right)}{u_{0}\left(x_{0}\right)}-m\right)\left\|u_{0}\right\|_{L^{2}\left(\mathbb{R}^{N}\right)}^{2}<-\gamma
\end{aligned}
$$

onde $\gamma=\frac{1}{2}\left(m-\frac{f\left(u_{0}\left(x_{0}\right)\right)}{u_{0}\left(x_{0}\right)}\right)\left\|u_{0}\right\|_{L^{2}\left(\mathbb{R}^{N}\right)}^{2}>0$. Em outras palavras, existe $\gamma>0$ tal que

$$
\int_{\mathbb{R}^{N}} f\left(u_{0}(z)\right) u_{0}(z) d z-m \int_{\mathbb{R}^{N}} u_{0}^{2}(z) d z<-\gamma
$$

Para a segunda integral em $(2.31)$, como $\int_{\mathbb{R}^{N}} u_{0}^{+}(x-y) u_{0}^{-}(x-y) d x=0$, temos

$$
\begin{aligned}
\int_{\mathbb{R}^{N}}\left[u_{0}^{2}(x-y)-\left(u_{0}^{+}\right)^{2}(x-y)\right] d x & =\int_{\mathbb{R}^{N}}\left\{\left[u_{0}^{+}(x-y)+u_{0}^{-}(x-y)\right]^{2}-\left(u_{0}^{+}\right)^{2}(x-y)\right\} d x \\
& =\int_{\mathbb{R}^{N}}\left[\left(u_{0}^{+}\right)^{2}(x-y)+\left(u_{0}^{-}\right)^{2}(x-y)-\left(u_{0}^{+}\right)^{2}(x-y)\right] d x \\
& =\int_{\mathbb{R}^{N}}\left(u_{0}^{-}\right)^{2}(x-y) d x .
\end{aligned}
$$

Afirmação 1: A integral $\int_{\mathbb{R}^{N}}\left(u_{0}^{-}\right)^{2}(x-y) d x \rightarrow 0$ quando $|y| \rightarrow \infty$. Com efeito, sendo $\phi_{1}, \cdots, \phi_{n}$ os autofunções que formam uma base para o espaço $E^{-}$, o Lema 16 e a hipótese $\left(V_{1}\right)$ garantem que, dado $\varepsilon>0$, para cada $i \in\{1, \cdots, n\}$, existe $M_{i}>0$ tal que

$$
\begin{aligned}
\left\langle u_{0}(x-y), \phi_{i}\right\rangle & =\frac{1}{2} \int_{\mathbb{R}^{N}} \nabla u_{0}(x-y) \cdot \nabla \phi_{i}(x) d x+\frac{1}{2} \int_{\mathbb{R}^{N}} V(x) u_{0}(x-y) \phi_{i}(x) d x \\
& <\varepsilon \text { quando }|y| \geq M_{i} .
\end{aligned}
$$


Tomando $\bar{M}=\max \left\{M_{1}, \cdots, M_{n}\right\}$, segue que, para todo $i \in\{1, \cdots, n\}$,

$$
\left\langle u_{0}(x-y), \phi_{i}\right\rangle<\varepsilon \text { quando }|y| \geq \bar{M} \text {. }
$$

Como $u_{0}^{-}(\cdot-y) \in E^{-}$é uma combinação linear das funções $\phi_{1}, \cdots, \phi_{n}$, digamos

$$
u_{0}^{-}(x-y)=\sum_{i=1}^{n} \xi_{i}(y) \phi_{i}(x)
$$

segue de (2.33) que existe $\bar{M}>0$ tal que, se $|y|>\bar{M}$, então

$$
\begin{aligned}
\left\|u_{0}^{-}(\cdot-y)\right\|^{2} & =\left\langle u_{0}(\cdot-y), u_{0}^{-}(\cdot-y)\right\rangle \\
& =\left\langle u_{0}(\cdot-y), \sum_{i=1}^{n} \xi_{i}(y) \phi_{i}(x)\right\rangle \\
& <\operatorname{\varepsilon n} \max \left\{\left|\xi_{1}(y)\right|, \cdots,\left|\xi_{n}(y)\right|\right\}
\end{aligned}
$$

Afirmação 2: Existe uma constante $C>0$, que não depende de $y$, tal que

$$
\max \left\{\left|\xi_{1}(y)\right|, \cdots,\left|\xi_{n}(y)\right|\right\}<C
$$

seja qual for $y \in \mathbb{R}^{N}$. Com efeito, sendo $\operatorname{dim} E^{-}<\infty$, pela equivalência de normas em espaços de dimensão finita, existe $D>0$, que não depende de $y$, tal que

$$
\left\|\sum_{i=1}^{n} \xi_{i}(y) \phi_{i}\right\|_{V_{\infty}}^{2} \geq D\left(\max \left\{\left|\xi_{1}(y)\right|, \cdots,\left|\xi_{n}(y)\right|\right\}\right)^{2}
$$

Portanto,

$$
\left\|u_{0}\right\|_{V_{\infty}}^{2} \geq\left\|u_{0}^{-}(\cdot-y)\right\|_{V_{\infty}}^{2}=\left\|\sum_{i=1}^{n} \xi_{i}(y) \phi_{i}\right\|_{V_{\infty}}^{2} \geq D\left(\max \left\{\left|\xi_{1}(y)\right|, \cdots,\left|\xi_{n}(y)\right|\right\}\right)^{2} .
$$

Isso demonstra a Afirmação 2, escolhendo $C=\frac{\left\|u_{0}\right\|_{V_{\infty}}^{2}}{\sqrt{D}}>0$. Agora, substituindo (2.35) em (2.34), obtemos

$$
\left\|u_{0}^{-}(\cdot-y)\right\|^{2}<\varepsilon n C
$$

para $|y| \geq \bar{M}$. Ora, sendo as normas $\|\cdot\|_{V_{\infty}} \mathrm{e}\|\cdot\|$ equivalentes em $E$, segue que $\left\|u_{0}^{-}(\cdot-y)\right\|_{V_{\infty}} \rightarrow 0$ quando $|y| \rightarrow \infty$. Assim,

$$
\int_{\mathbb{R}^{N}}\left(u_{0}^{-}\right)^{2}(x-y) d x \leq \frac{1}{V_{\infty}}\left\|u_{0}^{-}(\cdot-y)\right\|_{V_{\infty}}^{2} \rightarrow 0 \text { quando }|y| \rightarrow \infty,
$$


concluindo a demonstração da Afirmação 1. Substituindo (2.32) e (2.38) em (2.30), obtemos

$$
I(w) \leq \frac{1}{2}\|w\|^{2}\left\{\lambda^{2}\left[-\gamma+o_{|y|}(1)\right]+o_{R}(1)\right\}
$$

para $|y|$ e $R$ suficientemente grandes. Para concluir a demonstração do lema, como $w=\|w\|\left(\lambda u_{0}^{+}(\cdot-y)+v^{-}\right) \in M_{2}$ e, pela hipótese $\left(f_{3}\right), F$ uma função não negativa, temos

$$
\begin{aligned}
I(w) & =\frac{1}{2}\|w\|^{2}\left(\lambda^{2}\left\|u_{0}^{-}(\cdot-y)\right\|^{2}-\left\|v^{-}\right\|^{2}\right)-\int_{\mathbb{R}^{N}} F(w) d x \\
& \leq \frac{1}{2}\|w\|^{2}\left(\lambda^{2}\left\|u_{0}^{-}(\cdot-y)\right\|^{2}-\left\|v^{-}\right\|^{2}\right) .
\end{aligned}
$$

Uma vez que $\left\|\lambda u_{0}^{+}(\cdot-y)+v^{-}\right\|^{2}=1$, vale que

$$
\lambda^{2}\left\|u_{0}^{+}(\cdot-y)\right\|^{2}+\left\|v^{-}\right\|^{2}=1
$$

Então, a equação (2.40) se torna

$$
\begin{aligned}
I(w) & \leq \frac{1}{2}\|w\|^{2}\left(\lambda^{2}\left\|u_{0}^{-}(\cdot-y)\right\|^{2}-\left\|v^{-}\right\|^{2}\right) \\
& =\frac{1}{2}\|w\|^{2}\left(2 \lambda^{2}\left\|u_{0}^{-}(\cdot-y)\right\|^{2}-1\right) .
\end{aligned}
$$

Pela equivalência das normas e invariância por translação da norma $\|\cdot\|_{V_{\infty}}$, existe $C>0$, que não depende de $y$, tal que $2\left\|u_{0}^{+}(\cdot-y)\right\|^{2} \leq C\left\|u_{0}\right\|_{V_{\infty}}^{2}$. Assim, para

$$
\lambda^{2}<\frac{1}{C\left\|u_{0}\right\|_{V_{\infty}}^{2}} \leq \frac{1}{2\left\|u_{0}^{+}(\cdot-y)\right\|^{2}},
$$

temos $I(w)<0$, e o lema está provado para tais valores de $\lambda$. Se, por outro lado, $\lambda^{2} \geq \frac{1}{C\left\|u_{0}\right\|_{V_{\infty}}^{2}}:=k_{0}>0$, escolhemos $y \in \mathbb{R}^{N}$ com $|y|$ suficientemente grande para que

$$
-\gamma+o_{|y|}(1)<-\frac{\gamma}{2}
$$

Logo, a desigualdade (2.39) se torna

$$
I(w) \leq \frac{1}{2}\|w\|^{2}\left[-\lambda^{2} \frac{\gamma}{2}+o_{R}(1)\right] .
$$

Como $-\lambda^{2} \leq-k_{0}$, escolhendo $R$ suficientemente grande para que

$$
-k_{0} \frac{\gamma}{2}+o_{R}(1) \leq 0
$$


obtemos

$$
I(w) \leq \frac{1}{2}\|w\|^{2}\left[-\lambda^{2} \frac{\gamma}{2}+o_{R}(1)\right] \leq \frac{1}{2}\|w\|^{2}\left[-k_{0} \frac{\gamma}{2}+o_{R}(1)\right] \leq 0
$$

e o lema está provado para os valores de $\lambda$ tais que $\lambda^{2} \geq k_{0}$. Isso conclui a demonstração do lema.

A fim de obter a compacidade de uma sequência de Cerami para o funcional $I$, nos apoiaremos numa versão do Lema de Concentração e Compacidade devido a Lions [30].

Lema 18 (Splitting) Seja $\left(u_{n}\right)$ uma sequência limitada em $H^{1}\left(\mathbb{R}^{N}\right)$ tal que

$$
I\left(u_{n}\right) \rightarrow d>0 \quad \text { e } \quad\left\|I^{\prime}\left(u_{n}\right)\right\|\left(1+\left\|u_{n}\right\|\right) \rightarrow 0
$$

Então, a menos de subsequência, existe uma solução $u_{0}$ de $(P)$, um número $k \in \mathbb{N}, k$ funções $u_{1}, \cdots, u_{k}$ e $k$ sequências de pontos $\left(y_{n}^{j}\right) \in \mathbb{R}^{3}, 1 \leq j \leq k$, satisfazendo:

a) $u_{n} \rightarrow u_{0}$ em $H^{1}\left(\mathbb{R}^{N}\right)$ ou

b) $u_{j}$ são soluções não triviais of $\left(P_{\infty}\right)$;

c) $\left|y_{n}^{j}\right| \rightarrow+\infty$ e $\left|y_{n}^{j}-y_{n}^{i}\right| \rightarrow+\infty i \neq j$;

d) $I\left(u_{n}\right) \rightarrow I\left(u_{0}\right)+\sum_{i=1}^{k} I_{\infty}\left(u_{j}\right)$.

Demonstração. Uma vez que $f$ satisfaz $\left(f_{1}\right)$ e $\left(f_{4}\right)$, a prova deste resultado segue diretamente dos passos (2.11) e (2.46) a (2.55) da Proposição 2.31 de [10].

O resultado a seguir nos fornece uma comparação entre os níveis de energia dos funcionais $I$ e $I_{\infty}$. Este resultado combinado com o Lema 18 nos permite mostrar que toda sequência de Cerami para $I$ possui uma subsequência que converge forte.

Lema $19 c<c_{\infty}:=\inf \left\{I_{\infty}(w) ; w \in H^{1}\left(\mathbb{R}^{N}\right) \backslash\{0\}, I_{\infty}^{\prime}(w)=0\right\}$

Para demonstrá-lo, serão necessários alguns lemas auxiliares. Os dois primeiros podem ser encontrados em [7], nos Lemas 3.3 e 3.5. Por questão de completude, vamos acrescentar aqui a demosntração de cada um deles.

Lema 20 Existe $q \in\left(\frac{1}{2}, 1\right]$ com a seguinte propriedade: para qualquer $C_{3} \geq 1$, existe uma constante $C_{4}>0$ tal que a desigualdade

$$
|F(u+v)-F(u)-F(v)-f(u) v-f(v) u| \leq C_{4}|u v|^{2 q}
$$

é verdadeira para todo $u, v \in \mathbb{R}$ com $|u|,|v| \leq C_{3}$. 
Demonstração. Seja $p:=p_{1}$ e $q:=\min \left\{\frac{1}{4}(p+1), 1\right\}$. Observe que $\left(f_{4}\right)$ implies that $\left|f^{(k)}(u)\right| \leq C|u|^{p-k}$ se $0<|u| \leq C_{3}$. A prova da desigualdade requerida para $f(u)=$ $|u|^{p-1} u$, embora cansativa, é elementar. Reduzimos o caso geral a este caso especial da seguinte forma: Sejam $u, v>0$. Então,

$$
\begin{aligned}
\mid F(u+v)-F & (u)-F(v)-f(u) v-f(v) u \mid \\
& =\left|\int_{0}^{u} \int_{0}^{v} \int_{0}^{r} \int_{t}^{s+t} f^{\prime \prime \prime}(w) d w d t d r d s\right| \\
& \leq C \int_{0}^{u} \int_{0}^{v} \int_{0}^{r} \int_{t}^{s+t} w^{p-3} d w d t d r d s \\
& \leq C\left|(u+v)^{p+1}-u^{p+1}-v^{p+1}-u^{p} v-v^{p} u\right| \leq C(u v)^{2 q} .
\end{aligned}
$$

Isso conclui a demonstração do lema.

Lema 21 Se $\mu_{2}>\mu_{1} \geq 0$, existe $C>0$ tal que, para todo $x_{1}, x_{2} \in \mathbb{R}^{N}$,

$$
\int_{\mathbb{R}^{N}} e^{-\mu_{1}\left|x-x_{1}\right|} e^{-\mu_{2}\left|x-x_{2}\right|} d x \leq C e^{-\mu_{1}\left|x_{1}-x_{2}\right|} .
$$

Demonstração. Como $\mu_{1}\left|x_{1}-x_{2}\right|+\left(\mu_{2}-\mu_{1}\right)\left|x-x_{2}\right| \leq \mu_{1}\left(\left|x-x_{1}\right|+\left|x-x_{2}\right|\right)+\left(\mu_{2}-\right.$ $\left.\mu_{1}\right)\left|x-x_{2}\right|=\mu_{1}\left|x-x_{1}\right|+\mu_{2}\left|x-x_{2}\right|$, temos que

$$
\int_{\mathbb{R}^{N}} e^{-\mu_{1}\left|x-x_{1}\right|} e^{-\mu_{2}\left|x-x_{2}\right|} d x \leq \int_{\mathbb{R}^{N}} e^{-\mu_{1}\left|x_{1}-x_{2}\right|} e^{-\left(\mu_{2}-\mu_{1}\right)\left|x-x_{2}\right|} d x=C e^{-\mu_{1}\left|x_{1}-x_{2}\right|},
$$

e o lema segue.

Observamos que o conjunto $M$ definido no Teorema 15 é fechado e limitado e está contido em um espaço de dimensão finita, a saber, contido no espaço $\mathbb{R} u_{0}^{+}(\cdot-y) \oplus E^{-}$. Portanto, tal conjunto é compacto, o que implica que, para cada $y \in \mathbb{R}^{N}$, existe $w_{y}=v_{y}^{-}+t_{y} u_{0}^{+}(\cdot-y) \in M$ satisfazendo

$$
\max _{w \in M} I(w)=I\left(v_{y}^{-}+t_{y} u_{0}^{+}(\cdot-y)\right)
$$

O resultado a seguir mostra que os valores $t_{y}$ são limitados uniformemente em $y$ por constantes positivas desde que $|y|$ seja suficientemente grande.

Lema 22 Existem $A, B \in \mathbb{R}$, que não dependem de $y$, tais que $0<A \leq t_{y} \leq B$ para $|y|$ suficientemente grande. 
Demonstração. Como $w_{y}=v_{y}^{-}+t_{y} u_{0}^{+}(\cdot-y) \in M$ e o número $R>0$ dado pelo Teorema 15 não depende de $y$, tem-se

$$
R^{2} \geq\left\|w_{y}\right\|^{2}=\left\|v_{y}^{-}\right\|^{2}+t_{y}^{2}\left\|u_{0}^{+}(\cdot-y)\right\|^{2} \geq t_{y}^{2}\left(\left\|u_{0}(\cdot-y)\right\|^{2}-\left\|u_{0}^{-}(\cdot-y)\right\|^{2}\right) .
$$

Como foi provado em (2.37), se necessário, podemos tomar ainda maior o valor $|y|$ para que $\left\|u_{0}^{-}(\cdot-y)\right\|^{2} \leq \frac{C}{2}\left\|u_{0}\right\|_{V_{\infty}}^{2}$, onde $C>0$ é tal que $\left\|u_{0}(\cdot-y)\right\|^{2} \geq C\left\|u_{0}\right\|_{V_{\infty}}^{2}$ e não depende de $y$. Assim,

$$
R^{2} \geq t_{y}^{2}\left(\left\|u_{0}(\cdot-y)\right\|^{2}-\left\|u_{0}^{-}(\cdot-y)\right\|^{2}\right) \geq \frac{t_{y}^{2} C}{2}\left\|u_{0}\right\|_{V_{\infty}}^{2}
$$

ou seja, $t_{y} \leq \frac{2 R^{2}}{C\left\|u_{0}\right\|_{V_{\infty}}^{2}}:=B$. Por outro lado, pelo Lema 13 com $2<p<2^{*}$, para $\varepsilon>0$ ainda a ser escolhido, existe $C_{\varepsilon}>0$ tal que, se $u \in \tilde{E}^{+} \operatorname{com}\|u\|=\rho$, então

$$
I(u)=\frac{1}{2}\|u\|^{2}-\int_{\mathbb{R}^{N}} F(u) d x \geq \frac{1}{2} \rho^{2}-\varepsilon\|u\|_{L^{2}\left(\mathbb{R}^{N}\right)}^{2}-C_{\varepsilon}\|u\|_{L^{p}\left(\mathbb{R}^{N}\right)}^{p} .
$$

Pelas imersões de Sobolev e pela equivalência das normas, existem constantes $C_{3}, C_{4}>0$ que tornam (2.41) em

$$
I(u) \geq \frac{1}{2} \rho^{2}-\varepsilon C_{3}\|u\|^{2}-C_{4}\|u\|^{p}=\left(\frac{1}{2}-\varepsilon C_{3}\right) \rho^{2}-C_{4} \rho^{p} .
$$

Seja $\varepsilon>0$ tal que $D_{\varepsilon}:=\frac{1}{2}-\varepsilon C_{3}>0$. Escolhendo $\rho>0$ suficientemente pequeno de modo que

$$
D_{\varepsilon} \rho^{2}-C_{4} \rho^{p}>0
$$

isto é, $0<\rho<\left(\frac{D_{\varepsilon}}{C_{4}}\right)^{\frac{1}{p-2}}$, obtemos

$$
I(u) \geq D_{\varepsilon} \rho^{2}-C_{4} \rho^{p}:=\rho_{0}>0
$$

para todo $u \in \tilde{E}^{+} \operatorname{com}\|u\|=\rho$. Assim, tomamos $t>0$, que não depende de $y$, suficientemente pequeno de modo que $\left\|t u_{0}^{+}(\cdot-y)\right\|<R$ e $\left\|t u_{0}^{+}(\cdot-y)\right\|=\rho$ para concluir que

$$
I\left(t u_{0}^{+}(\cdot-y)\right) \geq \rho_{0}>0
$$

onde $\rho_{0}>0$ não depende de $y$. Consequentemente,

$$
I\left(v_{y}^{-}+t_{y} u_{0}^{+}(\cdot-y)\right)=\max _{w \in M} I(w) \geq I\left(t u_{0}^{+}(\cdot-y)\right)>\rho_{0},
$$


isto é,

$$
\frac{t_{y}^{2}}{2}\left\|u_{0}^{+}(\cdot-y)\right\|^{2}-\frac{1}{2}\left\|v_{y}\right\|^{2}-\int_{\mathbb{R}^{N}} F\left(v_{y}^{-}+t_{y} u_{0}^{+}(x-y)\right) d x=I\left(v_{y}^{-}+t_{y} u_{0}^{+}(\cdot-y)\right)>\rho_{0} .
$$

Logo, como $F$ é uma função não negativa,

$$
\frac{t_{y}^{2}}{2}\left\|u_{0}^{+}(\cdot-y)\right\|^{2}>\rho_{0},
$$

ou seja,

$$
t_{y}^{2}>\frac{2 \rho_{0}}{C\left\|u_{0}\right\|_{V_{\infty}}^{2}}:=A,
$$

onde $C>0$ é uma constante tal que $\left\|u_{0}^{+}(\cdot-y)\right\|^{2} \leq C\left\|u_{0}\right\|_{V_{\infty}}^{2}$ e não depende de $y$. O lema está provado.

Podemos, agora, provar o Lema 19.

Demonstração do Lema 19: Por simplicidade, vamos denotar $u_{0, y}(x)=u_{0}(x-y) \mathrm{e}$ $C>0$ denotará constantes possivelmente diferentes. Pela definição dos funcionais $I$ e $I_{\infty}$, temos

$$
\begin{aligned}
& I\left(v_{y}^{-}+t_{y} u_{0, y}^{+}\right)= \frac{t_{y}^{2}}{2}\left\|u_{0, y}^{+}\right\|^{2}-\frac{1}{2}\left\|v_{y}^{-}\right\|^{2}-\int_{\mathbb{R}^{N}} F\left(v_{y}^{-}+t_{y} u_{0, y}^{+}(x)\right) d x \\
& \leq \frac{t_{y}^{2}}{2}\left\|u_{0, y}\right\|^{2}-\int_{\mathbb{R}^{N}} F\left(t_{y} u_{0, y}\right) d x-\frac{t_{y}^{2}}{2}\left\|u_{0, y}^{-}\right\|^{2} \\
&+\int_{\mathbb{R}^{N}}\left(F\left(t_{y} u_{0, y}\right)-F\left(v_{y}^{-}+t_{y} u_{0, y}^{+}\right)\right) d x \\
& \leq I_{\infty}\left(t_{y} u_{0, y}\right)+\frac{t_{y}^{2}}{2} \int_{\mathbb{R}^{N}}\left(V(x)-V_{\infty}\right) u_{0, y}^{2}(x) d x \\
&+\int_{\mathbb{R}^{N}}\left(F\left(v_{y}^{-}-t_{y} u_{0, y}^{-}\right)+F\left(t_{y} u_{0, y}\right)-F\left(v_{y}^{-}+t_{y} u_{0, y}^{+}\right)\right) d x
\end{aligned}
$$

uma vez que $F$ é não negativa. Vamos estimar a última integral na desigualdade acima. Chamando $w_{y}^{-}=v_{y}^{-}-t_{y} u_{0, y}^{-}$, queremos estimar o termo

$$
\mathcal{I}_{y}=\left|\int_{\mathbb{R}^{N}}\left(F\left(w_{y}^{-}\right)+F\left(t_{y} u_{0, y}(x)\right)-F\left(w_{y}^{-}+t_{y} u_{0, y}(x)\right)\right) d x\right| .
$$

Para isso, temos

$$
\begin{aligned}
\mathcal{I}_{y} \leq & \int_{\mathbb{R}^{N}}\left|F\left(w_{y}^{-}\right)+F\left(t_{y} u_{0, y}\right)-F\left(w_{y}^{-}+t_{y} u_{0, y}\right)-f\left(w_{y}^{-}\right) t_{y} u_{0, y}-f\left(t_{y} u_{0, y}\right) w_{y}^{-}\right| d x \\
& +t_{y} \int_{\mathbb{R}^{N}}\left|f\left(w_{y}^{-}\right)\right|\left|u_{0, y}\right| d x+\int_{\mathbb{R}^{N}}\left|f\left(t_{y} u_{0, y}\right)\right|\left|w_{y}^{-}\right| d x .
\end{aligned}
$$


Como fizemos em (2.36), podemos mostrar que existe uma constante $C>0$, que não depende de $y$, tal que

$$
\left|w_{y}^{-}(x)\right| \leq C \sum_{i=1}^{k}\left|\phi_{i}(x)\right| \leq C \sum_{i=1}^{k} \sup _{\mathbb{R}^{N}}\left|\phi_{i}(x)\right|:=D,
$$

para todo $x \in \mathbb{R}^{N}$, uma vez que $w_{y}^{-}(x) \in M$ e, portanto, $\left\|w_{y}^{-}\right\|^{2} \leq R^{2}$. Sem perda de generalidade, podemos supor que $D>1$ também satisfaz $\left|u_{0, y}(x)\right| \leq D$, já que $u_{0}$ é contínua, radial e dacai a zero no infinito. Assim, podemos aplicar o Lema 20 e a hipótese $\left(f_{2}\right)$ e obter uma constante $C>0$ tal que

$$
\mathcal{I}_{y} \leq C t_{y}^{\mu} \int_{\mathbb{R}^{N}}\left|w_{y}^{-}\right|^{\mu}\left|u_{0, y}\right|^{\mu} d x+2 m t_{y} \int_{\mathbb{R}^{N}}\left|w_{y}^{-}\right|\left|u_{0, y}\right| d x
$$

em que $\mu=2 q>1$ e $q$ é dado pelo Lema 20. Agora, tomando $\xi=\lambda_{i}<0<V_{\infty}$ no Teorema 10, vemos que toda autofunção $\phi_{i}, i=1, \cdots, k$, satisfaz

$$
\left|\phi_{i}(x)\right| \leq C e^{-\delta|x|} \quad \text { para todo } \quad x \in \mathbb{R}^{N}
$$

para algum $\sqrt{V_{\infty}}<\delta<\sqrt{V_{\infty}-\lambda_{k}}$. Assim, de (2.45), tem-se

$$
\left|w_{y}^{-}(x)\right| \leq C e^{-\delta|x|} \quad \text { para todo } \quad x, y \in \mathbb{R}^{N}
$$

Como $u_{0}$ é a solução de $\left(P_{\infty}\right)$ dada por Berestycki e Lions em [4], temos que $\left|u_{0}(x)\right| \leq C e^{-\sqrt{V_{\infty}}|x|}$ para todo $x \in \mathbb{R}^{N}$. Segue do Lema 21 que

$$
\int_{\mathbb{R}^{N}}\left|w_{y}^{-}\right|\left|u_{0, y}\right| d x \leq C \int_{\mathbb{R}^{N}} e^{-\delta|x|} e^{-\sqrt{V_{\infty}}|x-y|} d x \leq C e^{-\sqrt{V_{\infty}}|y|}
$$

para alguma constante $C>0$. Da mesma maneira, pelo Lema 21, temos

$$
\int_{\mathbb{R}^{N}}\left|w_{y}^{-}\right|^{\mu}\left|u_{0, y}\right|^{\mu} d x \leq C \int_{\mathbb{R}^{N}} e^{-\delta \mu|x|} e^{-\mu \sqrt{V_{\infty}}|x-y|} d x \leq C e^{-\mu \sqrt{V_{\infty}}|y|} \leq C e^{-\sqrt{V_{\infty}}|y|}
$$

pois $\mu>1$. Esta estimativa combinada com (2.47) aplicada em (2.46) implica que

$$
\mathcal{I}_{y} \leq C e^{-\sqrt{V_{\infty}}|y|}
$$

onde usamos o Lema 22 para garantir que a constante $C>0$ não depende de $y$ (mais precisamente, não depende de $t_{y}$ ). Vamos estimar agora o termo

$$
\frac{t_{y}^{2}}{2} \int_{\mathbb{R}^{N}}\left(V(x)-V_{\infty}\right) u_{0, y}^{2}(x) d x .
$$


Ora, pela hipótese $\left(V_{4}\right)$, segue que

$$
\begin{aligned}
\frac{t_{y}^{2}}{2} \int_{\mathbb{R}^{N}}\left(V(x)-V_{\infty}\right) u_{0, y}^{2}(x) d x & =\frac{t_{y}^{2}}{2} \int_{\mathbb{R}^{N}}\left(V(x+y)-V_{\infty}\right) u_{0}^{2}(x) d x \\
& \leq-C e^{-\gamma|y|}
\end{aligned}
$$

para todo $y$, em que, novamente, $C>0$ não depende de $t_{y}$. Segue daí e de (2.48) que (2.43) pode ser escrito como

$$
I\left(v_{y}^{-}+t_{y} u_{0, y}^{+}\right) \leq I_{\infty}\left(t_{y} u_{0, y}\right)-C e^{-\gamma|y|}+C e^{-\sqrt{V_{\infty}}|y|}
$$

que, para $|y|$ suficientemente grande, pela hipótese $\left(V_{4}\right), 0<\gamma<\sqrt{V_{\infty}}$, se torna

$$
I\left(v_{y}^{-}+t_{y} u_{0, y}^{+}\right)<\max _{t \geq 0} I_{\infty}\left(t u_{0}\right)
$$

Pela hipótese $\left(f_{5}\right)$, o máximo $\max _{t \geq 0} I_{\infty}\left(t u_{0}\right)$ é atingido exatamente quando $t=1$, já que $u_{0}$ é ponto crítico não trivial do problema $\left(P_{\infty}\right)$. Este é o único momento em que usamos a hipótese $\left(f_{5}\right)$ no trabalho. Logo, como $u_{0}$ é solução de energia mínima, segue da definição do valor $c>0$ que

$$
c \leq \max _{w \in M} I(w)=I\left(v_{y}^{-}+t_{y} u_{0, y}^{+}\right)<c_{\infty},
$$

como queríamos demonstrar.

Finalmente, provaremos o resultado principal deste capítulo.

Prova do Teorema 12: Pelo Teorema 15, existe uma sequência de Cerami $\left(u_{n}\right)$ para o funcional $I$. Esta sequência é limitada pelo Lema 14. Assim, a menos de subsequência, $u_{n} \rightarrow u$ para alguma $u \in H^{1}\left(\mathbb{R}^{N}\right)$. Pelas imersões compactas de Sobolev, temos $I^{\prime}(u)=$ 0 . Consequentemente,

$$
I(u)=I(u)-\frac{1}{2} I^{\prime}(u) u=\int_{\mathbb{R}^{N}}\left(\frac{1}{2} f\left(u_{n}\right) u_{n}-F\left(u_{n}\right)\right) d x \geq 0,
$$

pela hipótese $\left(f_{3}\right)$. Com isso, se a convergência forte $u_{n} \rightarrow u$ não valesse, Lema de Concentração e Compacidade de Lions na sua versão do Lema 18 forneceria $k \geq 1$ soluções não triviais, $w_{1}, \cdots, w_{k}$, do problema $\left(P_{\infty}\right)$ tais que

$$
c+o_{n}(1)=I\left(u_{n}\right)=I(u)+\sum_{i=1}^{k} I_{\infty}\left(w_{i}\right)+o_{n}(1) \geq k c_{\infty}+o_{n}(1)
$$

Isto nos diz que $c \geq c_{\infty}$, contradizendo o Lema 19. Portanto, $u_{n} \rightarrow u$ e vale que $I(u)=c>0 \operatorname{com} I^{\prime}(u)=0$. Assim, $u \in H^{1}\left(\mathbb{R}^{N}\right)$ é uma solução não trivial do problema $(P)$, e o teorema está demonstrado. 


\section{Capítulo 3}

\section{Um problema não autônomo fortemente indefinido}

Neste capítulo, vamos estabelecer a existência de uma solução não trivial do problema elíptico

$$
-\Delta u+V(x) u=f(x, u), \quad u \in H^{1}\left(\mathbb{R}^{N}\right), \quad u \neq 0
$$

sem condições de periodicidade em $V$ e $f$, onde o potencial $V$ muda de sinal e a não linearidade $f$ é assintoticamente linear no infinito.

Problemas desse tipo tem sido estudado extensivamente para $V$ e $f$ periódicas na variável $x$, a fim de suprir a falta de compacidade em $\mathbb{R}^{N}$ (veja, por exemplo, $[24,27,28,36,47]$ e suas referências).

Stuart e Zhou no ilustre trabalho [46] a respeito de problemas assintoticamente lineares assumiram $V$ constante e $f$ radialmente simétrica em $x$. Explorando a condição de simetria radial, os autores foram capazes de recuperar a compacidade que é perdida quando se trata de problemas em domínios ilimitados e provaram a existência de uma solução positiva e radial de $(P)$.

Nossos resultados estão mais relacionados a [3], [25] e [46]. Diferentemente deles, o potencial $V$ é apenas contínuo e muda de sinal.

Assim como no capítulo anterior, embora não seja possível aplicar o teorema do passo da montanha, fazemos uso do clássico teorema de linking [38] sob a condição de Cerami [6], a fim de obter uma solução não trivial.

Vamos considerar o problema $(P) \operatorname{com} N \geq 3, u \in E:=H^{1}\left(\mathbb{R}^{N}\right)$ e $V$ um potencial satisfazendo as condições:

$\left(V_{1}\right) V \in C^{3}\left(\mathbb{R}^{N} \times \mathbb{R}, \mathbb{R}\right)$ e $-V_{0} \leq V(x) \leq V_{\infty}$, em que $V_{0}, V_{\infty}>0$ 
$\left(V_{2}\right) \lim _{|x| \rightarrow+\infty} V(x)=V_{\infty} ;$

$\left(V_{3}\right) 0 \notin \sigma(L)$ e $\inf \sigma(L)<0$, onde $\sigma(L)$ é o espectro do operador $L=-\Delta+V$;

$\left(V_{4}\right) V(x) \leq V_{\infty}-C_{1} e^{-\gamma|x|}$, em que $C_{1}>0$ e $0<\gamma<\sqrt{V_{\infty}}$.

Sobre a não linearidade $f$, vamos impor as seguintes condições.

$\left(f_{1}\right) f \in C^{3}\left(\mathbb{R}^{N} \times \mathbb{R}, \mathbb{R}\right)$ e $\lim _{s \rightarrow 0} \frac{f(x, s)}{s}=0$ uniformemente em $x \in \mathbb{R}^{N} ;$

$\left(f_{2}\right)$ Existem $a \in C\left(\mathbb{R}^{N}, \mathbb{R}^{+}\right)$e $h \in C(\mathbb{R}, \mathbb{R})$ tais que

$$
\lim _{|s| \rightarrow+\infty} \frac{|f(x, s)|}{|s|}=a(x) \quad \text { e } \quad \lim _{|x| \rightarrow+\infty} \frac{|f(x, s)|}{|s|}=h(s),
$$

$\mathrm{e}$

$$
\lim _{|x| \rightarrow \infty,|s| \rightarrow \infty} \frac{|f(x, s)|}{|s|}=\lim _{|s| \rightarrow \infty} h(s)=\lim _{|x| \rightarrow \infty} a(x)=a_{\infty}
$$

uniformemente em $x \in \mathbb{R}^{N}$. Além disso, $\frac{|f(x, s)|}{|s|} \leq a(x)$ e $a(x) \geq a_{\infty}>V_{\infty}$ para todo $s \neq 0$ e $x \in \mathbb{R}^{N}$;

$\left(f_{3}\right) h(s)<a_{\infty}$ para todo $s \in \mathbb{R}$

$\left(f_{4}\right)$ Sendo $F(x, s):=\int_{0}^{s} f(x, t) d t, \quad H(s):=\int_{0}^{s} h(t) t d t, \quad G(s):=\frac{1}{2} h(s) s^{2}-H(s)$ e $Q(x, s):=\frac{1}{2} f(x, s) s-F(x, s)$, então, para todo $s \in \mathbb{R} \backslash\{0\}$ e $x \in \mathbb{R}^{N}$,

$$
G(s)>0, \quad F(x, s) \geq 0, \quad Q(x, s)>0 \quad \text { e } \quad \lim _{|s| \rightarrow \infty} Q(x, s)=\infty
$$

uniformemente em $x \in \mathbb{R}^{N}$. Além disso, $H(s)<F(x, s)$ para todo $s>0$ e $x \in \mathbb{R}^{N}$;

$\left(f_{5}\right)$ Existem $C_{2}>0$ e $1<p_{1} \leq p_{2}$ tais que $p_{1}, p_{2}<2^{*}-1$ e

$$
\left|f^{(k)}(x, s)\right| \leq C_{2}\left(|s|^{p_{1}-k}+|s|^{p_{2}-k}\right)
$$

para $k \in\{0,1,2,3\}, s \in \mathbb{R}$ e $x \in \mathbb{R}^{N}$

$\left(f_{6}\right)$ A função $s \mapsto f(x, s) / s$ é crescente em $s \in(0,+\infty)$ para todo $x \in \mathbb{R}^{N}$.

Considere $f(x, s)=\frac{|s|^{3}}{1+c(x) s^{2}}$ para $x \in \mathbb{R}^{N}$ e $s \in \mathbb{R}$, onde $c \in C\left(\mathbb{R}^{N}, \mathbb{R}\right)$ é uma função positiva, $c(x) \rightarrow c_{\infty}>0$ quando $|x| \rightarrow \infty$ e $0<c_{0} \leq c(x)<c_{\infty}$, é um exemplo de uma função que satisfaz as condições $\left(f_{1}\right)-\left(f_{4}\right), \operatorname{com} a(x)=\frac{1}{c(x)}$ e $h(s)=\frac{s^{2}}{1+c_{\infty} s^{2}}$.

O principal resultado deste capítulo é o seguinte teorema. 
Teorema 23 Sob as condições $\left(V_{1}\right)-\left(V_{4}\right)$ e $\left(f_{1}\right)-\left(f_{6}\right)$, o problema $(P)$ possui uma solução fraca não trivial $u \in H^{1}\left(\mathbb{R}^{N}\right)$.

Observação 24 A condição $\left(f_{2}\right)$ implica que $h(s) \leq a_{\infty}$ para todo $s \in \mathbb{R}$. Porém, vamos precisar da desigualdade estrita $\left(f_{3}\right)$ no que segue.

\subsection{Estrutura variacional do problema}

Esta seção nada difere da seção do capítulo anterior, onde colocamos o problema na estrutura variacional. Por questão de completude e organização, vamos repetir os argumentos aqui.

Seja $E:=H^{1}\left(\mathbb{R}^{N}\right)$. O funcional energia $I: E \rightarrow \mathbb{R}$ associado à equação $(P)$ é dado por

$$
I(u)=\frac{1}{2} \int_{\mathbb{R}^{N}}\left(|\nabla u|^{2}+V(x) u^{2}\right) d x-\int_{\mathbb{R}^{N}} F(x, u) d x,
$$

com $u \in E$. É bem conhecido que, pelas hipóteses $\left(V_{2}\right)$ e $\left(V_{3}\right)$, o problema de autovalor

$$
-\Delta u+V(x) u=\lambda u, \quad u \in L^{2}\left(\mathbb{R}^{N}\right)
$$

possui uma sequência de autovalores tais que $\lambda_{1}<\lambda_{2} \leq \cdots \leq \lambda_{k}<0$ (see [17], Teorema 30, p. 150). Denotamos por $\phi_{i}$ a autofunção correspondendo ao autovalor $\lambda_{i}$, para $i \in\{1,2, \cdots, k\}$, em $H^{1}\left(\mathbb{R}^{N}\right)$. Considerando

$$
E^{-}:=\operatorname{span}\left\{\phi_{i}, i=1,2, \cdots, k\right\} \quad \text { e } \quad E^{+}:=\left(E^{-}\right)^{\perp}
$$

vemos que $E=E^{+} \oplus E^{-}$. De acordo com o Teorema 8 , o espectro essencial de $-\Delta+V$ é o intervalo $\left[V_{\infty},+\infty\right)$, e isto implica que $\operatorname{dim} E^{-}<\infty$. Tendo feitas essas considerações, toda função $u \in E$ pode ser escrita como $u=u^{+}+u^{-}$unicamente, onde $u^{+} \in E^{+} \mathrm{e}$ $u^{-} \in E^{-}$. Assim, usando os argumentos do Lema 1.2 de [9], podemos introduzir um novo produto interno $\langle\cdot, \cdot\rangle$ em $E$, a saber, a forma bilinear definida por

$$
\langle u, v\rangle=\left\{\begin{array}{cl}
\int_{\mathbb{R}^{N}}(\nabla u \cdot \nabla v+V(x) u v) d x & \text { se } u, v \in E^{+} \\
-\int_{\mathbb{R}^{N}}(\nabla u \cdot \nabla v+V(x) u v) d x & \text { se } u, v \in E^{-} \\
0 & \text { se } u \in E^{+} \text {e } v \in E^{-},
\end{array}\right.
$$


tal que a norma correspondente $\|\cdot\|$ é equivalente a $\|\cdot\|_{E}$, a norma usual em $E=H^{1}\left(\mathbb{R}^{N}\right)$. Além disso, o funcional $I$ pode ser escrito como

$$
I(u)=\frac{1}{2}\left\|u^{+}\right\|^{2}-\frac{1}{2}\left\|u^{-}\right\|^{2}-\int_{\mathbb{R}^{N}} F(x, u) d x
$$

para toda função $u=u^{+}+u^{-} \in E$. Chamamos a atenção ao fato de que, como $\lambda_{i} \neq 0$ para todo $i \in\{1,2, \cdots, k\}$, seque de (3.1) e da definição de $\phi_{i}$ que

$$
\int_{\mathbb{R}^{N}} u^{+}(x) v^{-}(x) d x=0
$$

para toda função $u^{+} \in E^{+}$e $v^{-} \in E^{-}$.

Os resultados do capítulo anterior, que lidamos com um problema autônomo, são provados de maneira semelhante neste capítulo. Vamos apresentá-los com suas devidas adaptações.

\subsection{Limitação das sequências de Cerami}

Como consequência direta das hipóteses $\left(f_{1}\right)$ e $\left(f_{2}\right)$, segue o seguinte lema auxiliar.

Lema 25 Sob as hipóteses $\left(f_{1}\right) e\left(f_{2}\right)$, dado $\varepsilon>0$, existe $C_{\varepsilon}>0$ tal que, para $2 \leq p \leq 2^{*}$,

$$
|F(x, s)| \leq \varepsilon|s|^{2}+C_{\varepsilon}|s|^{p} \quad e \quad|f(x, s)| \leq \varepsilon|s|+C_{\varepsilon}|s|^{p-1}
$$

para todo $s \in \mathbb{R}$ e para todo $x \in \mathbb{R}^{N}$.

Demonstração. Pelas hipóteses $\left(f_{1}\right)$ e $\left(f_{2}\right)$, dado $\varepsilon>0$, existem $R, \delta>0$ tais que $R>\delta$ com

$$
|f(x, t)| \leq \varepsilon|t| \text { sempre que }|t|<\delta \text { e para todo } x \in \mathbb{R}^{N}
$$

e

$$
|f(x, t)-a(x) t| \leq \varepsilon|t| \text { sempre que }|t|>R \text { e para todo } x \in \mathbb{R}^{N} \text {. }
$$

A desigualdade 3.3 implica que

$$
|f(x, t)| \leq \varepsilon|t|+a_{0}|t| \text { sempre que }|t|>R \text { e para todo } x \in \mathbb{R}^{N}
$$


onde $a_{0}=\sup _{\mathbb{R}^{N}}|a(x)|$. Para os valores de $t$ tais que $|t|>R$, vale $|t|<\frac{|t|^{p-1}}{R^{p-2}}$. Portanto, (3.4) se torna

$$
|f(x, t)| \leq \varepsilon|t|+\frac{a_{0}}{R^{p-2}}|t|^{p-1} \text { sempre que }|t|>R \text { e para todo } x \in \mathbb{R}^{N}
$$

Pela hipótese $\left(f_{2}\right)$, temos

$$
|f(x, t)| \leq|a(x) t| \leq a_{0}|t| \text { sempre que } \delta \leq|t| \leq R \text { e para todo } x \in \mathbb{R}^{N} .
$$

Logo, para valores de $t$ em que $\delta \leq|t| \leq R$, obtemos

$$
|f(x, t)| \leq \frac{a_{0}}{\delta^{p-2}}|t|^{p-1} \text { sempre que } \delta \leq|t| \leq R \text { e para todo } x \in \mathbb{R}^{N}
$$

Segue de (3.2), (3.5) e (3.6) que

$$
|f(x, t)| \leq \varepsilon|t|+\left(\frac{a_{0}}{R^{p-2}}+\frac{a_{0}}{\delta^{p-2}}\right)|t|^{p-1} \text { para todo } t \in \mathbb{R} \text { e para todo } x \in \mathbb{R}^{N} .
$$

Chamando $C_{\varepsilon}:=\left(\frac{a_{0}}{R^{p-2}}+\frac{a_{0}}{\delta^{p-2}}\right)$ e, em seguida, integrando a desigualdade (3.7) de $0 \mathrm{a}$ $s$, obtemos

$$
|F(x, s)| \leq \frac{\varepsilon}{2}|s|^{2}+\frac{C_{\varepsilon}}{p}|s|^{p} \text { para todo } s \in \mathbb{R}
$$

concluindo a demonstração do lema.

Notamos que, se $\left(v_{n}\right)$ é uma sequência limitada em $E$, então $\left(v_{n}\right)$ satisfaz apenas uma das condições a seguir.

(i) vanishing: para todo $r>0, \limsup _{n \rightarrow \infty} \sup _{y \in \mathbb{R}^{N}} \int_{B(y, r)}\left|v_{n}\right|^{2} d x=0$;

(ii) nonvanishing: existem $r, \eta>0$ e uma sequência $\left(y_{n}\right) \subset \mathbb{R}^{N}$ tais que

$$
\limsup _{n \rightarrow \infty} \int_{B\left(y_{n}, r\right)}\left|v_{n}\right|^{2} d x>\eta .
$$

Lema 26 Seja $\left(u_{n}\right) \subset$ E uma sequência tal que $I\left(u_{n}\right) \rightarrow c>0$ e $\left\|I^{\prime}\left(u_{n}\right)\right\|_{E^{*}}\left(1+\left\|u_{n}\right\|\right) \rightarrow$ 0 quando $n \rightarrow+\infty$. Então, $\left(u_{n}\right)$ possui uma subsequência limitada.

Demonstração. Vamos supor que $\left\|u_{n}\right\| \rightarrow \infty$ e obter uma contradição. Para isso, considere $v_{n}=\frac{u_{n}}{\left\|u_{n}\right\|}$ e observe que $\left\|v_{n}\right\|=1$. Suponha que valha (ii) para a sequência $\left(v_{n}\right)$. Escreva $f(x, s)=a(x) s+(f(x, s)-a(x) s)=a(x) s+f_{\infty}(x, s)$ e considere $\varphi \in$ 
$C_{0}^{\infty}\left(\mathbb{R}^{N}\right)$. Pela equivalência das normas $\|\cdot\|$ e $\|\cdot\|_{E}$, existem constantes $C_{1}, C_{2}>0$ tais que

$$
\|w\| \leq C_{1}\|w\|_{H^{1}\left(\mathbb{R}^{N}\right)} \leq C_{2}\|w\|, \text { para toda função } w \in E .
$$

Seja $\left(y_{n}\right) \subset \mathbb{R}^{N}$ a sequência dada pela hipótese $(i i)$. Como a sequência $\left(u_{n}\right)$ é de Cerami e considerando $\varphi_{n}(x)=\varphi\left(x-y_{n}\right)$, temos de $(3.8)$ que

$$
\left|I^{\prime}\left(u_{n}\right) \varphi_{n}\right| \leq\left\|I^{\prime}\left(u_{n}\right)\right\|_{E^{*}}\left\|\varphi_{n}\right\| \leq C_{1}\left\|I^{\prime}\left(u_{n}\right)\right\|_{E^{*}}\left\|\varphi_{n}\right\|_{E}=C_{1}\left\|I^{\prime}\left(u_{n}\right)\right\|_{E^{*}}\|\varphi\|_{E} \rightarrow 0
$$

Logo,

$$
\begin{aligned}
o_{n}(1) & =\frac{1}{\left\|u_{n}\right\|} I^{\prime}\left(u_{n}\right) \varphi_{n}=\left\langle v_{n}^{+}-v_{n}^{-}, \varphi_{n}\right\rangle-\int_{\mathbb{R}^{N}} \frac{f\left(x, u_{n}\right)}{\left\|u_{n}\right\|} \varphi_{n} d x \\
& =\left\langle v_{n}^{+}-v_{n}^{-}, \varphi_{n}\right\rangle-\int_{\mathbb{R}^{N}} a(x) v_{n} \varphi_{n} d x-\int_{\mathbb{R}^{N}} \frac{f_{\infty}\left(x, u_{n}\right)}{\left\|u_{n}\right\|} \varphi_{n} d x \\
& =\left\langle v_{n}^{+}-v_{n}^{-}, \varphi_{n}\right\rangle-\int_{\mathbb{R}^{N}} a(x) v_{n} \varphi_{n} d x-\int_{\mathbb{R}^{N}} \frac{f_{\infty}\left(x, u_{n}\right)}{u_{n}} v_{n} \varphi_{n} d x
\end{aligned}
$$

Considere $\tilde{v}_{n}(x)=v_{n}\left(x+y_{n}\right)$ e $\tilde{u}_{n}(x)=u_{n}\left(x+y_{n}\right)$. Observe que $\left(\tilde{v}_{n}\right)$ é limitada em $E$. Assim, a menos de subsequência,

$$
\begin{array}{rc}
\tilde{v}_{n} \rightarrow \tilde{v} & \text { em } E, \\
\tilde{v}_{n} \rightarrow \tilde{v} & \text { em } L_{l o c}^{2}\left(\mathbb{R}^{N}\right), \\
\left|\tilde{v}_{n}(x)\right| \leq h_{0}(x) & \text { q.t.p. em } K,
\end{array}
$$

para alguma função $h_{0} \in L^{1}(K)$, onde $K=\operatorname{supp}(\varphi)$. Pelas hipóteses $\left(V_{1}\right)$ e $\left(f_{2}\right)$ e lembrando que $f_{\infty}(x, s)=f(x, s)-a(x) s$, temos

$$
\left|\frac{f_{\infty}\left(x+y_{n}, \tilde{u}_{n}\right)}{\tilde{u}_{n}} \tilde{v}_{n} \varphi\right| \leq C h_{0}(x) \varphi \in L^{1}(K) .
$$

Observamos que $\tilde{v} \neq 0$. Da hipótese $\left(f_{2}\right)$ e de (3.11), segue do Teorema da Convergência Dominada de Lebesgue que

$$
\int_{\mathbb{R}^{N}} \frac{f_{\infty}\left(x, u_{n}\right)}{u_{n}} v_{n} \varphi_{n} d x \rightarrow 0 \text { quando } n \rightarrow \infty .
$$

Assim, de (3.9) e (3.12), o Teorema da Mudança de Variável nos fornece

$$
\begin{aligned}
o_{n}(1)= & \int_{\mathbb{R}^{N}}\left(\nabla \tilde{v}_{n}^{+} \nabla \varphi+V\left(x+y_{n}\right) \tilde{v}_{n}^{+} \varphi\right) d x+\int_{\mathbb{R}^{N}}\left(\nabla \tilde{v}_{n}^{-} \nabla \varphi+V\left(x+y_{n}\right) \tilde{v}_{n}^{-} \varphi\right) d x \\
& -\int_{\mathbb{R}^{N}} a\left(x+y_{n}\right) \tilde{v}_{n} \varphi d x .
\end{aligned}
$$


Caso 1: $\left|y_{n}\right| \rightarrow \infty$. Neste caso, pela hipótese $\left(V_{2}\right), V\left(x+y_{n}\right) \rightarrow V_{\infty}$ e, pela hipótese $\left(f_{2}\right), a\left(x+y_{n}\right) \rightarrow a_{\infty}$ q.t.p. em $\mathbb{R}^{N}$. Assim,

$$
\begin{aligned}
o_{n}(1)= & \int_{K}\left(\nabla \tilde{v}_{n}^{+} \nabla \varphi+\left(V_{\infty}+o_{n}(1)\right) \tilde{v}_{n}^{+} \varphi\right) d x+\int_{K}\left(\nabla \tilde{v}_{n}^{-} \nabla \varphi+\left(V_{\infty}+o_{n}(1)\right) \tilde{v}_{n}^{-} \varphi\right) d x \\
& -\int_{K}\left(a_{\infty}+o_{n}(1)\right) \tilde{v}_{n} \varphi d x .
\end{aligned}
$$

Portanto, para toda função $\varphi \in C_{0}^{\infty}\left(\mathbb{R}^{N}\right)$, tomando $n \rightarrow \infty$ em (3.13), obtemos

$$
\int_{\mathbb{R}^{N}}\left(\nabla\left(\tilde{v}^{+}+\tilde{v}^{-}\right) \nabla \varphi+V_{\infty}\left(\tilde{v}^{+}+\tilde{v}^{-}\right) \varphi\right) d x-\int_{\mathbb{R}^{N}} a_{\infty} \tilde{v} \varphi d x=0,
$$

isto é, $\tilde{v} \neq 0$ é solução fraca do problema

$$
-\Delta \tilde{v}+V_{\infty} \tilde{v}=a_{\infty} \tilde{v} \text { em } \mathbb{R}^{N}
$$

Uma vez que $V_{\infty}<a_{\infty}$ e não existe autofunção do laplaciano em $\mathbb{R}^{N}$, isso é um absurdo. Logo, (ii) não pode valer quando $\left|y_{n}\right| \rightarrow \infty$.

Caso 2: $\left(y_{n}\right)$ é uma sequência limitada. Neste caso, de (3.10),

$$
0 \neq|\tilde{v}(x)|=\lim _{n \rightarrow \infty}\left|\tilde{v}_{n}(x)\right|=\lim _{n \rightarrow \infty} \frac{\left|\tilde{u}_{n}(x)\right|}{\left\|\tilde{u}_{n}\right\|}
$$

q.t.p. em $\Omega \subset B(0,1)$, onde $\mu(\Omega)>0$. Visto que $\left\|\tilde{u}_{n}\right\| \rightarrow \infty$, temos $\tilde{u}_{n}(x) \rightarrow \infty$ q.t.p. em $\Omega$. Logo, as hipóteses $\left(V_{1}\right),\left(f_{4}\right)$ e o Lema de Fatou nos fornecem

$$
\begin{aligned}
\liminf _{n \rightarrow \infty} \int_{\mathbb{R}^{N}}\left(\frac{1}{2} f\left(x, u_{n}\right) u_{n}-F\left(x, u_{n}\right)\right) d x & \geq \liminf _{n \rightarrow \infty} \int_{\Omega}\left(\frac{1}{2} f\left(x-y_{n}, \tilde{u}_{n}\right) \tilde{u}_{n}-F\left(x-y_{n}, \tilde{u}_{n}\right)\right) d x \\
& \geq \int_{\Omega} \liminf _{n \rightarrow \infty}\left(\frac{1}{2} f\left(x-y_{n}, \tilde{u}_{n}\right) \tilde{u}_{n}-F\left(x-y_{n}, \tilde{u}_{n}\right)\right) d x \\
& =+\infty .
\end{aligned}
$$

Todavia, isso contradiz o fato de

$$
\int_{\mathbb{R}^{N}}\left(\frac{1}{2} f\left(x, u_{n}\right) u_{n}-F\left(x, u_{n}\right)\right) d x=I\left(u_{n}\right)-\frac{1}{2} I^{\prime}\left(u_{n}\right) u_{n}=c+o_{n}(1) .
$$

Logo, não vale o Caso 2 quando a sequência $\left(y_{n}\right)$ é limitada. Isso mostra que não pode ocorrer a hipótese $(i i)$ para a sequência $\left(v_{n}\right)$. Suponha, agora, que valha a hipótese $(i)$ para a sequência $\left(v_{n}\right)$. Ora, como a sequência $\left(u_{n}\right)$ é de Cerami,

$$
o_{n}(1)=I^{\prime}\left(u_{n}\right) \frac{u_{n}^{+}}{\left\|u_{n}\right\|^{2}}=\frac{1}{\left\|u_{n}\right\|} I^{\prime}\left(u_{n}\right) v_{n}^{+}=\left\|v_{n}^{+}\right\|^{2}-\int_{\mathbb{R}^{N}}\left(\frac{f\left(x, u_{n}\right)}{u_{n}} v_{n} v_{n}^{+}\right) d x
$$


e

$$
o_{n}(1)=I^{\prime}\left(u_{n}\right) \frac{u_{n}^{-}}{\left\|u_{n}\right\|^{2}}=\frac{1}{\left\|u_{n}\right\|} I^{\prime}\left(u_{n}\right) v_{n}^{-}=-\left\|v_{n}^{-}\right\|^{2}-\int_{\mathbb{R}^{N}}\left(\frac{f\left(x, u_{n}\right)}{u_{n}} v_{n} v_{n}^{-}\right) d x
$$

Subtraindo de (3.15) a equação (3.16), temos

$$
\begin{aligned}
o_{n}(1) & =\left\|v_{n}^{+}\right\|^{2}+\left\|v_{n}^{-}\right\|^{2}-\int_{\mathbb{R}^{N}} a(x)\left(\frac{f\left(u_{n}\right)}{u_{n}} v_{n}\left(v_{n}^{+}-v_{n}^{-}\right)\right) d x \\
& =\left\|v_{n}\right\|^{2}-\int_{\mathbb{R}^{N}} a(x)\left(\frac{f\left(u_{n}\right)}{u_{n}} v_{n}\left(v_{n}^{+}-v_{n}^{-}\right)\right) d x \\
& =1-\int_{\mathbb{R}^{N}}\left(\frac{f\left(x, u_{n}\right)}{u_{n}} v_{n}\left(v_{n}^{+}-v_{n}^{-}\right)\right) d x .
\end{aligned}
$$

Então, necessariamente, quando $n \rightarrow \infty$,

$$
\int_{\mathbb{R}^{N}}\left(\frac{f\left(x, u_{n}\right)}{u_{n}} v_{n}\left(v_{n}^{+}-v_{n}^{-}\right)\right) d x \rightarrow 1
$$

Dado $0<\varepsilon<\frac{1}{2} \mu_{0}$, onde $\mu_{0}>0$ é a constante tal que

$$
\|w\|^{2} \geq \mu_{0}\|w\|_{L^{2}\left(\mathbb{R}^{N}\right)}^{2}
$$

para toda função $w \in E$, pela hipótese $\left(f_{1}\right)$, existe $\delta>0$ tal que, para todo $x \in \mathbb{R}^{N}$,

$$
\frac{|f(x, s)|}{|s|} \leq \varepsilon \text { sempre que } 0 \neq|s|<\delta .
$$

Para cada $n \in \mathbb{N}$, considere o conjunto

$$
\tilde{\Omega}_{n}=\left\{x \in \mathbb{R}^{N} ;\left|u_{n}(x)\right|<\delta\right\}
$$

Então, a desigualdade de Hölder garante que

$$
\begin{aligned}
\int_{\tilde{\Omega}_{n}}\left(\frac{f\left(x, u_{n}\right)}{u_{n}} v_{n}\left(v_{n}^{+}-v_{n}^{-}\right)\right) d x & \leq \varepsilon \int_{\tilde{\Omega}_{n}}\left|v_{n} \| v_{n}^{+}-v_{n}^{-}\right| d x \\
& \leq \varepsilon a_{\infty}\left(\left\|v_{n}\right\|_{L^{2}\left(\mathbb{R}^{N}\right)}\left\|v_{n}^{+}\right\|_{L^{2}\left(\mathbb{R}^{N}\right)}+\left\|v_{n}\right\|_{L^{2}\left(\mathbb{R}^{N}\right)}\left\|v_{n}^{-}\right\|_{L^{2}\left(\mathbb{R}^{N}\right)}\right) \\
& \leq 2 \varepsilon\left\|v_{n}\right\|_{L^{2}\left(\mathbb{R}^{N}\right)}^{2} \\
& \leq \frac{2 \varepsilon}{\mu_{0}}\left\|v_{n}\right\|^{2}=\frac{2 \varepsilon}{\mu_{0}}<1 .
\end{aligned}
$$

A partir de (3.17), concluímos que

$$
\liminf _{n \rightarrow \infty} \int_{\mathbb{R}^{N} \backslash \tilde{\Omega}_{n}}\left(\frac{f\left(x, u_{n}\right)}{u_{n}} v_{n}\left(v_{n}^{+}-v_{n}^{-}\right)\right) d x>0 .
$$


Das hipóteses $\left(V_{1}\right)$ e $\left(f_{2}\right)$, do fato de $\frac{f(\cdot, \cdot)}{|\cdot|}$ ser uma função limitada e da desigualdade de Hölder com expoente $\frac{p}{2}>1$, vale que

$$
\int_{\mathbb{R}^{N} \backslash \tilde{\Omega}_{n}}\left(\frac{f\left(x, u_{n}\right)}{u_{n}} v_{n}\left(v_{n}^{+}-v_{n}^{-}\right)\right) d x \leq C \mu\left(\mathbb{R}^{N} \backslash \tilde{\Omega}_{n}\right)^{\frac{p-2}{p}}\left\|v_{n}\right\|_{L^{p}\left(\mathbb{R}^{N}\right)}^{\frac{2}{p}} .
$$

Desde que vale $(i)$, o Lema de Lions garante que $\left\|v_{n}\right\|_{L^{p}\left(\mathbb{R}^{N}\right)} \rightarrow 0$. Portanto, a menos de subsequência, segue de (3.18) que

$$
\mu\left(\mathbb{R}^{N} \backslash \tilde{\Omega}_{n}\right) \rightarrow \infty \quad \text { quando } \quad n \rightarrow \infty
$$

Pela hipótese $\left(f_{3}\right)$, existe $R>0$ tal que, se $|s|>R$,

$$
\frac{1}{2} f(x, s) s-F(x, s)>1
$$

para todo $x \in \mathbb{R}^{N}$. Sem perda de generalidade, podemos supor $0<\delta<R$. Para cada $n \in \mathbb{N}$, considere $A_{n}:=\left\{x \in \mathbb{R}^{N} ;\left|u_{n}(x)\right|>R\right\}$. Então, pela hipótese $\left(f_{4}\right)$,

$$
\begin{aligned}
c+o_{n}(1) & =I\left(u_{n}\right)-\frac{1}{2} I^{\prime}\left(u_{n}\right) u_{n} \\
& =\int_{\mathbb{R}^{N}}\left(\frac{1}{2} f\left(x, u_{n}\right) u_{n}-F\left(x, u_{n}\right)\right) d x \\
& \geq \int_{A_{n}}\left(\frac{1}{2} f\left(x, u_{n}\right) u_{n}-F\left(x, u_{n}\right)\right) d x>\mu\left(A_{n}\right)
\end{aligned}
$$

o que implica que a sequência $\left(\mu\left(A_{n}\right)\right)$ é limitada. Considere também $B_{n}:=\{x \in$ $\left.\mathbb{R}^{N} ; \delta \leq\left|u_{n}(x)\right| \leq R\right\}$. Como $B_{n}=\left(\mathbb{R}^{N} \backslash \tilde{\Omega}_{n}\right) \backslash A_{n}$, temos

$$
\mu\left(\mathbb{R}^{N} \backslash \tilde{\Omega}_{n}\right)=\mu\left(A_{n}\right)+\mu\left(B_{n}\right)
$$

Segue de (3.20) e da limitação da sequência $\left(\mu\left(A_{n}\right)\right)$ que

$$
\mu\left(B_{n}\right) \rightarrow \infty
$$

Afirmamos que $\bar{\delta}:=\inf _{\substack{s \in[\delta, R] \\ x \in \mathbb{R}^{N}}}\left(\frac{1}{2} f(x, s) s-F(x, s)\right)>0$. Com efeito, seja $\left(x_{n}, s_{n}\right) \in \mathbb{R}^{N} \times$ $[\delta, R]$ uma sequência satisfazendo

$$
\lim _{n \rightarrow \infty}\left(\frac{1}{2} f\left(x_{n}, s_{n}\right) s_{n}-F\left(x_{n}, s_{n}\right)\right)=\bar{\delta}
$$


Ora, como o intervalo $[\delta, R]$ é compacto, podemos sempre supor que $s_{n} \rightarrow s_{0} \in[\delta, R]$. Se $x_{n} \rightarrow x_{0}$, pela continuidade das funções $f$ e $F$, segue da hipótese $\left(f_{4}\right)$ que $\bar{\delta}>0$. Por outro lado, se $\left|x_{n}\right| \rightarrow \infty$, escrevendo

$$
\frac{1}{2} f\left(x_{n}, s_{n}\right) s_{n}-F\left(x_{n}, s_{n}\right)=\frac{1}{2}\left(\frac{f\left(x_{n}, s_{n}\right)}{s_{n}}-h\left(s_{n}\right)\right) s_{n}^{2}-\left(F\left(x_{n}, s_{n}\right)-H\left(s_{n}\right)\right)+G\left(s_{n}\right)
$$

onde $G\left(s_{n}\right)=\frac{1}{2} h\left(s_{n}\right) s_{n}^{2}-H\left(s_{n}\right)$, segue dos limites uniformes em $\left(f_{2}\right)$ e da hipótese $\left(f_{4}\right)$ que

$$
\bar{\delta}=\lim _{n \rightarrow \infty}\left(\frac{1}{2} f\left(x_{n}, s_{n}\right) s_{n}-F\left(x_{n}, s_{n}\right)\right)=G\left(s_{0}\right)>0,
$$

como havíamos afirmado. Assim, de $(3.21)$ e da hipótese $\left(f_{4}\right)$,

$$
\begin{aligned}
\int_{\mathbb{R}^{N}}\left(\frac{1}{2} f\left(x, u_{n}\right) u_{n}-F\left(x, u_{n}\right)\right) d x & \geq \int_{B_{n}}\left(\frac{1}{2} f\left(x, u_{n}\right) u_{n}-F\left(x, u_{n}\right)\right) d x \\
& \geq \bar{\delta} \mu\left(B_{n}\right) \rightarrow \infty
\end{aligned}
$$

Isso novamente contradiz o fato de

$$
\int_{\mathbb{R}^{N}}\left(\frac{1}{2} f\left(x, u_{n}\right) u_{n}-F\left(x, u_{n}\right)\right) d x=I\left(u_{n}\right)-\frac{1}{2} I^{\prime}\left(u_{n}\right) u_{n}=c+o_{n}(1) .
$$

Logo, $(i)$ também não pode ocorrer para a sequência $\left(v_{n}\right)$. Mas isso é um absurdo. Concluímos que, a menos de subsequência, $\left(u_{n}\right)$ é limitada.

\subsection{Uma solução não trivial}

Dedicamos esta seção à prova de que o funcional $I$ satisfaz a geometria do Teorema de Linking, provado em [29], Proposição 2.10, e à demonstração do teorema principal deste capítulo.

Teorema 27 (Teorema de Linking para sequências de Cerami) Seja $E=E^{+} \oplus E^{-} u m$ espaço de Banach com dim $E^{-}<\infty$. Sejam $R>\rho>0$ e $u \in E^{+}$um elemento fixo tal que $\|u\|=\rho$. Considere

$$
\begin{aligned}
M & :=\left\{w=t u+v^{-}:\|w\| \leq R, t \geq 0, v^{-} \in E^{-}\right\} \\
M_{0} & :=\left\{w=t u+v^{-}: v^{-} \in E^{-},\|w\|=R, t \geq 0 \text { ou }\|w\| \leq R, t=0\right\} \\
N_{\rho} & :=\left\{w \in E^{+}:\|w\|=\rho\right\}
\end{aligned}
$$


Seja $I \in C^{1}(E, \mathbb{R})$ tal que

$$
b:=\inf _{N_{\rho}} I>a:=\max _{M_{0}} I .
$$

Então, $c \geq b$ e existe uma sequência de Cerami no nível c para o funcional I, onde

$$
c:=\inf _{\gamma \in \Gamma} \max _{w \in M} I(\gamma(w)), \quad \Gamma:=\left\{\gamma \in C(M, E):\left.\gamma\right|_{M_{0}}=I d\right\}
$$

Considere o problema limite

$$
-\Delta w+V_{\infty} w=h(w) w \text { em } \mathbb{R}^{N}
$$

e o funcional energia associado a ele dado por

$$
I_{\infty}(w)=\frac{1}{2} \int_{\mathbb{R}^{N}}\left(|\nabla w|^{2}+V_{\infty} w^{2}\right) d x-\int_{\mathbb{R}^{N}} H(w) d x
$$

para $w \in E$, onde $H(s)=\int_{0}^{s} h(t) t d t$. Como $V_{\infty}<a_{\infty}$, é provado em [4] que o problema $\left(P_{\infty}\right)$ possui uma solução clássica, radialmente simétrica e positiva $u_{0} \in E$.

Para $R>0$ e $y \in \mathbb{R}^{N}$, considere

$$
M=\left\{w=t u_{0}^{+}(\cdot-y)+v^{-} ;\|w\| \leq R, t \geq 0, v^{-} \in E^{-}\right\}
$$

e

$$
M_{0}=\left\{w=t u_{0}^{+}(\cdot-y)+v^{-} ; v^{-} \in E^{-},\|w\|=R, t \geq 0 \text { ou }\|w\| \leq R, t=0\right\} .
$$

A prova do seguinte lema é uma leve adaptação da prova do Lema 17. Por isso, vamos destacar apenas os passos em que tais adaptações aparecem.

Lema 28 Existem $R>0$ e $y \in \mathbb{R}^{N}$, com $R,|y|$ suficientemente grandes, tais que

$$
\left.I\right|_{M_{0}} \leq 0
$$

Demonstração. Como $M_{0}=M_{1} \cup M_{2}$ é uma união disjunta, onde

$$
M_{1}=\left\{w=t u_{0}^{+}(\cdot-y)+v^{-} ; v^{-} \in E^{-},\|w\| \leq R, t=0\right\}
$$

e

$$
M_{2}=\left\{w=t u_{0}^{+}(\cdot-y)+v^{-} ; v^{-} \in E^{-},\|w\|=R, t>0\right\},
$$


e $I(w) \leq 0$ sempre que $w \in M_{1}$, sejam $R>0$ e $w \in M_{2}$ com $\|w\|=R$. Escrevendo $w=\|w\| \frac{w}{\|w\|}=\|w\| u(w)=\|w\|\left(\lambda(w) u_{0}^{+}(\cdot-y)+v^{-}(w)\right)$, temos

$$
\begin{aligned}
I(w) & =I(\|w\| u(w))=\frac{1}{2}\|w\|^{2} \lambda^{2}(w)\left\|u_{0}^{+}(\cdot-y)\right\|^{2}-\frac{1}{2}\|w\|^{2}\left\|v^{-}(w)\right\|^{2}-\int_{\mathbb{R}^{N}} F(x,\|w\| u(w)) d x \\
& =\frac{1}{2}\|w\|^{2}\left\{\lambda^{2}(w)\left\|u_{0}^{+}(\cdot-y)\right\|^{2}-\left\|v^{-}(w)\right\|^{2}-2 \int_{\mathbb{R}^{N}} \frac{F(x, R u(w))}{(R u(w))^{2}} u^{2}(w) d x\right\}
\end{aligned}
$$

Escreveremos $\lambda, u$ e $v^{-}$ao invés de $\lambda(w), u(w)$ e $v^{-}(w)$, respectivamente. Pela hipótese $\left(f_{4}\right), \lim _{s \rightarrow \infty} \frac{F(x, s)}{s^{2}}=\frac{1}{2} a(x)$ e $\frac{F(x, s)}{s^{2}} \leq \frac{1}{2} a(x)$ para todo $s \neq 0$ e $x \in \mathbb{R}^{N}$. Segue da hipótese $\left(f_{4}\right)$ que

$$
\left|\frac{F(x, R u)}{(R u)^{2}} u^{2}\right| \leq \frac{C}{2} u^{2} \in L^{1}\left(\mathbb{R}^{N}\right)
$$

e, pelo Teorema da Convergência Dominada de Lebesgue,

$$
\lim _{R \rightarrow \infty} \int_{\mathbb{R}^{N}}\left(\frac{F(x, R u)}{(R u)^{2}}-\frac{a(x)}{2}\right) u^{2} d x=0
$$

para todo $u \in E$ tal que $\|u\|=1$. Visto que o conjunto $M_{2}$ está contido em um subespaço de dimensão finita de $E, w=R u \in M_{2}$ com $\|u\|=1$, o limite (3.22) é uniforme em $u$ (veja Apêndice A, Lema 44). Segue do fato de $a(x) \geq a_{\infty}$ que

$$
\begin{aligned}
I(w)= & \frac{1}{2}\|w\|^{2}\left\{\lambda^{2}\left\|u_{0}^{+}(\cdot-y)\right\|^{2}-\left\|v^{-}\right\|^{2}-\int_{\mathbb{R}^{N}} a(x) u^{2} d x+o_{R}(1)\right\} \\
\leq & \frac{1}{2}\|w\|^{2}\left\{\lambda^{2}\left\|u_{0}^{+}(\cdot-y)\right\|^{2}-\left\|v^{-}\right\|^{2}-a_{\infty} \int_{\mathbb{R}^{N}}\left(\lambda u_{0}^{+}(x-y)+v^{-}\right)^{2} d x+o_{R}(1)\right\} \\
= & \frac{1}{2}\|w\|^{2}\left\{\lambda^{2}\left\|u_{0}^{+}(\cdot-y)\right\|^{2}-\left\|v^{-}\right\|^{2}-a_{\infty} \lambda^{2} \int_{\mathbb{R}^{N}}\left(u_{0}^{+}\right)^{2}(x-y) d x\right. \\
& \left.-a_{\infty} \int_{\mathbb{R}^{N}}\left(v^{-}\right)^{2} d x+o_{R}(1)\right\} \\
\leq & \frac{1}{2}\|w\|^{2}\left\{\lambda^{2}\left[\left\|u_{0}^{+}(\cdot-y)\right\|^{2}-a_{\infty} \int_{\mathbb{R}^{N}}\left(u_{0}^{+}\right)^{2}(x-y) d x\right]+o_{R}(1)\right\} .
\end{aligned}
$$

Pela hipótese $\left(f_{3}\right)$ e pelo fato de $u_{0}$ ser ponto crítico do funcional $I$, temos que

$$
\left\|u_{0}^{+}(\cdot-y)\right\|^{2} \leq \int_{\mathbb{R}^{N}} h\left(u_{0}(x-y)\right) u_{0}^{2}(x-y) d x .
$$

Substituindo (3.24) em (3.23) e, em seguida, somando e subtraindo a integral $a_{\infty} \int_{\mathbb{R}^{N}} u_{0}^{2}(x-y) d x$, obtemos da hipótese $\left(V_{1}\right)$,

$I(w) \leq \frac{1}{2}\|w\|^{2}\left\{\lambda^{2}\left[\int_{\mathbb{R}^{N}} h\left(u_{0}(x-y)\right) u_{0}^{2}(x-y) d x-a_{\infty} \int_{\mathbb{R}^{N}}\left(u_{0}^{+}\right)^{2}(x-y) d x\right]+o_{R}(1)\right\}$ 


$$
\begin{aligned}
= & \frac{1}{2}\|w\|^{2}\left\{\lambda ^ { 2 } \left[\int_{\mathbb{R}^{N}} h\left(u_{0}(x-y)\right) u_{0}^{2}(x-y) d x-a_{\infty} \int_{\mathbb{R}^{N}} u_{0}^{2}(x-y) d x\right.\right. \\
& \left.\left.+a_{\infty} \int_{\mathbb{R}^{N}}\left[u_{0}^{2}(x-y)-\left(u_{0}^{+}\right)^{2}(x-y)\right] d x\right]+o_{R}(1)\right\} \\
= & \frac{1}{2}\|w\|^{2}\left\{\lambda ^ { 2 } \left[\int_{\mathbb{R}^{N}} h\left(u_{0}(z)\right) u_{0}^{2}(z) d z-a_{\infty} \int_{\mathbb{R}^{N}} u_{0}^{2}(z) d z\right.\right. \\
& \left.\left.+a_{\infty} \int_{\mathbb{R}^{N}}\left[u_{0}^{2}(x-y)-\left(u_{0}^{+}\right)^{2}(x-y)\right] d x\right]+o_{R}(1)\right\} .
\end{aligned}
$$

Como foi feito para se obter (2.32), usando a hipótese $\left(f_{3}\right)$, existe $\gamma>0$ tal que

$$
\int_{\mathbb{R}^{N}}\left(h\left(u_{0}(z)\right)-a_{\infty}\right) u_{0}^{2}(z) d z<-\gamma
$$

De (2.38), temos

$$
\int_{\mathbb{R}^{N}} a_{\infty}\left[u_{0}^{2}(x-y)-\left(u_{0}^{+}\right)^{2}(x-y)\right] d x \rightarrow 0 \text { quando }|y| \rightarrow \infty .
$$

Substituindo (3.26) e (3.27) em (3.25), obtemos

$$
I(w) \leq \frac{1}{2}\|w\|^{2}\left\{\lambda^{2}\left[-\gamma+o_{|y|}(1)\right]+o_{R}(1)\right\}
$$

para $|y|$ e $R$ suficientemente grandes. Isso conclui a demonstração do lema.

A fim de obter a compacidade de uma sequência de Cerami para o funcional $I$, vamos nos basear novamente no Lema 18.

Observação 29 Podemos também demonstrar o Lema 22 com as mesmas ideias contidas lá, e, por este motivo, não vamos incluir aqui sua demonstração. Portanto, existem $A, B \in \mathbb{R}$, que não dependem de $y$, tais que $0<A \leq t_{y} \leq B$ para $|y|$ suficientemente grande. Aqui, ty é tal que $\max _{w \in M} I(w)=I\left(v_{y}^{-}+t_{y} u_{0}^{+}(\cdot-y)\right)$.

Estamos aptos a provar a estimativa de energia dada pelo resultado a seguir.

Lema $30 c<c_{\infty}:=\inf \left\{I_{\infty}(w) ; w \in H^{1}\left(\mathbb{R}^{N}\right) \backslash\{0\}, I_{\infty}^{\prime}(w)=0\right\}$

Demonstração. Embora a demonstração siga de maneira análoga àquela do capítulo anterior, há aqui uma pequena adaptação. Quando somamos e subtraímos termos para se obter a estimativa

$$
\begin{aligned}
I\left(v_{y}^{-}+t_{y} u_{0, y}^{+}\right) \leq & I_{\infty}\left(t_{y} u_{0, y}\right)+\frac{t_{y}^{2}}{2} \int_{\mathbb{R}^{N}}\left(V(x)-V_{\infty}\right) u_{0, y}^{2}(x) d x \\
& +\int_{\mathbb{R}^{N}}\left(F\left(v_{y}^{-}-t_{y} u_{0, y}^{-}\right)+F\left(t_{y} u_{0, y}\right)-F\left(v_{y}^{-}+t_{y} u_{0, y}^{+}\right)\right) d x
\end{aligned}
$$


precisamos somar e subtrair a integral $\int_{\mathbb{R}^{N}}\left[H\left(t_{y} u_{0, y}\right)-F\left(t_{y} u_{0, y}\right)\right] d x$, que é negativa pela hipótese $\left(f_{4}\right)$. A partir daí, a prova segue os mesmos passos da do Lema 19.

Podemos demonstrar o principal resultado deste capítulo.

Prova do Teorema 23: Segue diretamente dos mesmos passos da demonstração do Teorema 12. 


\section{Capítulo 4}

\section{Uma equação de Schrödinger quasilinear com um potencial indefinido}

Neste capítulo, estudamos a existência de solução de onda estacionária para a equação de Schrödinger quasilinear

$$
i \frac{\partial z}{\partial t}=-\Delta z+W(x) z-l\left(|z|^{2}\right) z-k\left[\Delta \rho\left(|z|^{2}\right)\right] \rho^{\prime}\left(|z|^{2}\right) z,
$$

onde $z: \mathbb{R} \times \mathbb{R}^{3} \rightarrow \mathbb{C}, W: \mathbb{R}^{3} \rightarrow \mathbb{R}$ é um potencial dado, $k$ é uma constante real e $l$ e $\rho$ são funções reais. A propagação de um curto pulso de laser ultra intenso de auto-focagem pode ser modelada por esta equação assim como outros fenômenos na física dos plasmas e na mecânica dos fluídos (veja [31] e suas referências).

Nosso objetivo é mostrar a existência de uma solução estacionária não trivial de (4.1) com $\rho(t)=t$ e $k=1$, o que nos leva a considerar a equação quasilinear

$$
-\Delta u+V(x) u-u \Delta\left(u^{2}\right)=g(x, u) \quad \text { em } \mathbb{R}^{3}, \quad u \in H^{1}\left(\mathbb{R}^{3}\right) \backslash\{0\}
$$

onde $g$ tem um crescimento subcrítico e $V$ é um potencial que muda de sinal, mas não é necessariamente simétrico, podendo ser ilimitado inferiormente.

Neste cenário, muito pouco é encontrado na literatura. Indicamos o trabalho [33], que lida com um problema similar, onde $V$ é radialmente simétrica e pode mudar de sinal. Mais recentemente, em [49], sob a condição de que $V$ é uma função satisfazendo $\inf _{\mathbb{R}^{N}} V(x)>-\infty$, os autores aplicam uma abordagem dual com o teorema do passo da montanha para obter infinitas soluções para o problema não autônomo $(P) \operatorname{com} g(x, u)$ 
ímpar na variável $u$. Outro trabalho recente em equação de Schrödinger quasilinear com potencial que muda de sinal é [20], o qual ataca o problema $(P)$ sob hipóteses muito similares às nossas. Todavia, a existência de uma solução fraca não trivial para o problema não autônomo é garantida acrescentando-se um termo de perturbação não trivial a não linearidade, o que simplifica drasticamente os argumentos, pois os autores não precisam lidar com questões de compacidade.

Existem muitos trabalhos concernentes ao problema $(P) \operatorname{com} V$ satisfazendo $\inf _{\mathbb{R}^{N}} V(x)>$ 0 e $g$ uma não linearidade que possui crescimento subcrítico ou crítico (veja, por exemplo, [8, 21, 31, 37, 41, 42, 48]). O caso de massa zero, $V \equiv 0$, foi estudado em [8]. O caso em que o potencial converge a zero foi considerado em [1].

Para provar a existência de uma solução para a equação $(P)$, usamos métodos variacionais combinados com o princípio de concentração e compacidade de Lions [30]. A fim de lidar com o potencial $V$ que muda de sinal, fomos beneficiados com algumas ideias de [22]. Os argumentos que aplicamos impõem uma restrição na dimensão do espaço euclidiano, que ficará clara no decorrer do trabalho.

Seja $2^{*}=6$. As condições sobre a não linearidade $g$ são as seguintes.

$\left(g_{1}\right) g \in C^{1}\left(\mathbb{R}^{3} \times \mathbb{R}, \mathbb{R}\right)$ e $\lim _{s \rightarrow 0} \frac{g(x, s)}{s}=0$ uniformemente em $x \in \mathbb{R}^{3} ;$

$\left(g_{2}\right)$ Existem $g_{\infty} \in C\left(\mathbb{R}^{3}, \mathbb{R}^{+}\right), h \in C^{1}(\mathbb{R}, \mathbb{R})$ e $\eta_{1} \in\left(3,2^{*}-1\right]$ tais que

$$
\lim _{|s| \rightarrow+\infty} \frac{|g(x, s)|}{|s|^{\eta_{1}}}=g_{\infty}(x) \quad \text { e } \quad \lim _{|x| \rightarrow+\infty} g(x, s)=h(s),
$$

e

$$
\lim _{|x| \rightarrow \infty,|s| \rightarrow \infty} \frac{|g(x, s)|}{|s|^{\eta_{1}}}=\lim _{|s| \rightarrow \infty} \frac{h(s)}{|s|^{\eta_{1}}}=\lim _{|x| \rightarrow \infty} g_{\infty}(x)=a_{\infty}>0
$$

uniformemente em $x \in \mathbb{R}^{3}$;

$\left(g_{3}\right)$ Existe $\mu>4$ tal que, se $G(x, s):=\int_{0}^{s} g(x, t) d t$, então, para todo $s \neq 0$ e $x \in \mathbb{R}^{3}$,

$$
0<\mu G(x, s) \leq g(x, s) s
$$

$\left(g_{4}\right)$ A função $s \mapsto \frac{h(s)}{s^{3}}$ é não decrescente para $s \in(0,+\infty)$;

$\left(g_{5}\right)$ Existem constantes $a_{1}, a_{2}>0$ tais que

$$
\left|g_{s}(x, s)\right| \leq a_{1}+a_{2}|s|^{2^{*}-2}
$$

para todo $x \in \mathbb{R}^{3}$ e $s \in \mathbb{R}$. 
A função $g(x, s)=\frac{3|s|^{11}}{1+a(x) s^{6}}$, onde $a \in C\left(\mathbb{R}^{3}, \mathbb{R}\right)$ é uma função positva com $a(x) \rightarrow$ $a_{\infty}>0$ quando $|x| \rightarrow \infty$ é um exemplo de uma função que satisfaz as condições $\left(g_{1}\right)-$ $\left(g_{4}\right)$, tomando $\eta_{1}=5, g_{\infty}(x)=\frac{3}{a(x)}$ e $\frac{h(s)}{s^{5}}=\frac{3 s^{6}}{1+a_{\infty} s^{6}}$.

Sobre o potencial $V: \mathbb{R}^{3} \rightarrow \mathbb{R}$, escrevendo $V(x)=V^{+}(x)-V^{-}(x)$, onde $V^{ \pm}(x):=$ $\max \{ \pm V(x), 0\}$, impomos as seguintes condições.

$\left(V_{1}\right) \lim _{|x| \rightarrow \infty} V(x)=V_{\infty}>0$

$\left(V_{2}\right) V(x) \leq V_{\infty}$ para todo $x \in \mathbb{R}^{3}$ e $V(x)<V_{\infty}$ em algum conjunto de medida positiva;

$\left(V_{3}\right)$ Se $\mu_{1}=\mu / 2>2$ e $S$ é a melhor constante da imersão de Sobolev $D^{1,2}\left(\mathbb{R}^{3}\right) \hookrightarrow$ $L^{2^{*}}\left(\mathbb{R}^{3}\right)$, então

$$
\left\|V^{-}\right\|_{L^{3 / 2}\left(\mathbb{R}^{3}\right)}<S\left(\frac{\mu_{1}-2}{\mu_{1}-1}\right)<S
$$

O resultado principal deste capítulo é o seguinte teorema.

Teorema 31 Sob as condições $\left(g_{1}\right)-\left(g_{4}\right)$ e $\left(V_{1}\right)-\left(V_{3}\right)$, o problema $(P)$ possui uma solução fraca não trivial $u \in H^{1}\left(\mathbb{R}^{3}\right)$.

\subsection{Resultados principais}

Uma das dificuldades de se trabalhar com o problema $(P)$ usando métodos variacionais é que o funcional energia associado a este problema não está bem definido em $H^{1}\left(\mathbb{R}^{3}\right)$. Mais precisamente, o funcional $I: H^{1}\left(\mathbb{R}^{3}\right) \rightarrow \mathbb{R}$ associado à equação $(P)$, dado por

$$
J(u)=\frac{1}{2} \int_{\mathbb{R}^{3}}\left(|\nabla u|^{2}+V(x) u^{2}\right) d x+\int_{\mathbb{R}^{3}} u^{2}|\nabla u|^{2} d x-\int_{\mathbb{R}^{3}} G(x, u) d x,
$$

para $u \in H^{1}\left(\mathbb{R}^{3}\right)$, não está bem definido, pois $\int_{\mathbb{R}^{3}} u^{2}|\nabla u|^{2} d x$ não é finito para toda função $u \in H^{1}\left(\mathbb{R}^{3}\right)$. A fim de contornar esta dificuldade, usamos a seguinte mudança de variável introduzida por Liu, Wang e Wang em [31] e Colin e Jeanjean em [8], a saber, $u=f(w)$, onde $f$ é definida por

$$
f^{\prime}(t)=\frac{1}{\sqrt{1+2 f^{2}(t)}} \quad \text { em } \quad[0,+\infty) \quad \text { e } \quad f(t)=-f(-t) \quad \text { em } \quad(-\infty, 0]
$$

e satisfaz as seguintes propriedades, as quais podem ser encontradas em [8]:

$\left(f_{1}\right) f \in C^{\infty}(\mathbb{R})$ é unicamente determinada e invertível; 
$\left(f_{2}\right)\left|f^{\prime}(t)\right| \leq 1$ para todo $t \in \mathbb{R}$

$\left(f_{3}\right)|f(t)| \leq|t|$ para todo $t \in \mathbb{R}$

$\left(f_{4}\right) \frac{f(t)}{t} \rightarrow 1$ quando $t \rightarrow 0 ;$

$\left(f_{5}\right)|f(t)| \geq C|t|^{1 / 2}$ para algum $C>0$ e para todo $t \geq 1$;

$\left(f_{6}\right) \frac{f^{2}(t)}{2} \leq f(t) f^{\prime}(t) t \leq f^{2}(t)$ para todo $t \in \mathbb{R} ;$

$\left(f_{7}\right)$ Se $\lambda<1$, então $f^{2}(\lambda t) \geq \lambda^{2} f^{2}(t)$ para todo $t \in \mathbb{R}$;

$\left(f_{8}\right) \frac{f(t)}{2} \leq t f^{\prime}(t) \leq f(t)$ para todo $t \in \mathbb{R} ;$

$\left(f_{9}\right)\left|f(t) f^{\prime}(t)\right| \leq \frac{1}{\sqrt{2}}$ para todo $t \in \mathbb{R}$

$\left(f_{10}\right)|f(t)| \leq 2^{1 / 4}|t|^{1 / 2}$ para todo $t \in \mathbb{R}$

$\left(f_{11}\right) \frac{f(t)}{\sqrt{t}} \rightarrow 2^{1 / 4}$ quando $t \rightarrow+\infty$;

Como consequência das propriedades da função $f$, o seguinte lema é válido. Sua prova pode ser encontrada em [15], no Corolário 2.3.

Lema 32 A função $f$ satisfaz:

i) $t \mapsto f(t) f^{\prime}(t) t^{-1}$ é decrescente para $t>0$;

ii) $t \mapsto f^{3}(t) f^{\prime}(t) t^{-1}$ é crescente para $t>0$.

Após a mudança de variável $u=f(w)$ em (4.2), obtemos

$$
I(w)=\frac{1}{2} \int_{\mathbb{R}^{3}}\left(|\nabla w|^{2}+V(x) f^{2}(w)\right) d x-\int_{\mathbb{R}^{3}} G(x, f(w)) d x
$$

e o funcional $I$ está associado ao problema

$$
-\Delta w+V(x) f(w) f^{\prime}(w)=g(x, f(w)) f^{\prime}(w) \text { em } \mathbb{R}^{3}
$$

para $w \in H^{1}\left(\mathbb{R}^{3}\right)$. Notamos que, se $w$ é uma solução fraca para o problema $\left(P_{f}\right)$, então $u=f(w)$ é uma solução fraca para o problema $(P)$ (veja [8]). Assim, nos concentraremos em resolver o problema $\left(P_{f}\right)$, isto é, encontrar uma solução para a equação $I^{\prime}(w)=0$ para $w \in H^{1}\left(\mathbb{R}^{3}\right)$. Pelas hipóteses $\left(g_{1}\right),\left(g_{2}\right),\left(V_{1}\right)$ e pelas propriedades $\left(f_{1}\right)-\left(f_{3}\right)$, o funcional 
I está bem definido. Para se obter a compacidade de uma sequência de Cerami para o funcional $I$, considere o problema limite

$$
-\Delta w+V_{\infty} f(w) f^{\prime}(w)=h(f(w)) f^{\prime}(w) \quad \text { em } \mathbb{R}^{3}
$$

para $w \in H^{1}\left(\mathbb{R}^{3}\right)$, cujo funcional energia associado é dado por

$$
I_{\infty}(w)=\frac{1}{2} \int_{\mathbb{R}^{3}}\left(|\nabla w|^{2}+V_{\infty} f^{2}(w)\right) d x-\int_{\mathbb{R}^{3}} H(f(w)) d x
$$

onde $H(s):=\int_{0}^{s} h(t) d t$. Também temos que $I_{\infty}$ está bem definido. $\operatorname{Por}\left(g_{1}\right),\left(g_{2}\right),\left(f_{1}\right)$ e $\left(V_{1}\right)-\left(V_{3}\right)$ os funcionais $I$ e $I_{\infty}$ são de classe $C^{1}$. Considere o conjunto

$$
\mathcal{N}_{\infty}=\left\{w \in H^{1}\left(\mathbb{R}^{3}\right) \backslash\{0\} ; I_{\infty}^{\prime}(w) w=0\right\}
$$

No decorrer deste capítulo, $C$ denota constantes positivas possivelmente diferentes. A demonstração do seguinte resultado pode ser encontrada em [22], no Lema 2.1.

Lema 33 A forma quadrática

$$
u \mapsto \int_{\mathbb{R}^{N}}\left(|\nabla u|^{2}+V^{+}(x) u^{2}\right) d x
$$

define uma norma em $H^{1}\left(\mathbb{R}^{3}\right)$, a qual é equivalente à norma usual $\|\cdot\|_{H^{1}\left(\mathbb{R}^{3}\right)}$.

Provaremos que toda função positiva $w \in H^{1}\left(\mathbb{R}^{3}\right)$ pode ser unicamente projetada no conjunto $\mathcal{N}_{\infty}$. O resultado a seguir vem nesta direção.

Lema 34 Dado $w \in H^{1}\left(\mathbb{R}^{3}\right)$ com $w>0$, existe único $t_{w}>0$ tal que $t_{w} w \in \mathcal{N}_{\infty}$. Além disso, $I_{\infty}\left(t_{w} w\right)=\max _{t \geq 0} I_{\infty}(t w)$.

Demonstração. Seja $w \in H^{1}\left(\mathbb{R}^{3}\right) \operatorname{com} w>0$. Para cada $t \geq 0$, considere $\xi(t)=I_{\infty}(t w)$. Então, pela definição do funcional $I_{\infty}$ e como $f(0)=0$, segue que $\xi(0)=0$. Temos

$$
\begin{aligned}
\xi(t) & =\frac{1}{2} \int_{\mathbb{R}^{3}}\left(|\nabla t w|^{2}+V_{\infty} f^{2}(t w)\right) d x-\int_{\mathbb{R}^{3}} H(f(t w)) d x \\
& \geq \frac{t^{2}}{2}\left(\int_{\mathbb{R}^{3}}|\nabla w|^{2} d x-2 \int_{\mathbb{R}^{3}} \frac{H(f(t w))}{f^{2}(t w)} \frac{f^{2}(t w)}{t^{2}} d x\right) .
\end{aligned}
$$

A propriedade $\left(f_{3}\right)\left(\right.$ ou $\left.\left(f_{4}\right)\right)$ e as hipóteses $\left(g_{1}\right)$ e $\left(g_{2}\right)$ garantem que

$$
\xi(t) \geq \frac{t^{2}}{2}\left(\int_{\mathbb{R}^{3}}|\nabla w|^{2} d x-o_{t}(1)\right),
$$


quando $t \rightarrow 0^{+}$. Assim, para $t>0$ suficientemente pequeno, tem-se $\xi(t)>0$. Por outro lado, note que, pela propriedade $\left(f_{3}\right)$, existe uma constante $C>0$ tal que

$$
\begin{aligned}
\xi(t) & =\frac{1}{2} \int_{\mathbb{R}^{3}}\left(|\nabla t w|^{2}+V_{\infty} f^{2}(t w)\right) d x-\int_{\mathbb{R}^{3}} H(f(t w)) d x \\
& \leq t^{2}\left(\frac{C}{2}\|w\|_{H^{1}\left(\mathbb{R}^{3}\right)}^{2}-\int_{\mathbb{R}^{3}} \frac{H(f(t w))}{t^{2}} d x\right) .
\end{aligned}
$$

Afirmamos que

$$
\frac{H(f(t))}{t^{2}} \rightarrow+\infty \quad \text { quando } \quad t \rightarrow+\infty
$$

De fato, pela hipótese $\left(g_{2}\right), h(t) \rightarrow+\infty$ quando $t \rightarrow+\infty$. Logo, como pela propriedade $\left(f_{11}\right), f(t) \rightarrow+\infty$, temos que $H(f(t)) \rightarrow+\infty$ quando $t \rightarrow+\infty$. Portanto, a hipótese $\left(g_{2}\right)$ nos fornece

$$
\lim _{t \rightarrow+\infty} \frac{H(f(t))}{f^{4}(t)}=\lim _{t \rightarrow+\infty} \frac{h(f(t))}{4 f^{3}(t)}=\lim _{t \rightarrow+\infty} \frac{h(f(t))}{4 f^{\eta_{1}}(t)} f(t)^{\eta_{1}-3}=+\infty .
$$

Disto e da propriedade $\left(f_{11}\right)$, segue que

$$
\frac{H(f(t))}{t^{2}}=\frac{H(f(t))}{f^{4}(t)} \frac{f^{4}(t)}{(\sqrt{t})^{4}} \rightarrow+\infty \quad \text { quando } \quad t \rightarrow+\infty
$$

como afirmamos. Assim, (4.7) e (4.8) implicam que

$$
\xi(t) \rightarrow-\infty \text { quando } t \rightarrow+\infty
$$

Segue de (4.6), (4.10) e da hipótese $\left(g_{1}\right)$ que a função $\xi$ assume um máximo global $t_{w}>0$. Disto, $\xi^{\prime}\left(t_{w}\right)=0$, isto é, $t_{w} w \in \mathcal{N}_{\infty}$. Agora, a fim de provar a unicidade, mostraremos que, se $w>0$, então a equação

$$
I_{\infty}^{\prime}(t w) t w=0
$$

admite apenas uma solução para $t>0$. Com efeito, observe que a equação (4.11) é equivalente a

$$
\int_{\mathbb{R}^{3}}|\nabla w|^{2} d x+\int_{\mathbb{R}^{3}} V_{\infty} \frac{f(t w) f^{\prime}(t w)}{t} w d x=\int_{\mathbb{R}^{3}} \frac{h(f(t w)) f^{\prime}(t w)}{t} w d x
$$

Portanto, pelo Lema 32, a função em $t>0$ do lado esquerdo da igualdade (4.12) é decrescente, e a função do lado direito, pela hipótese $\left(g_{4}\right)$, é crescente. Isto conclui a demonstração do lema.

Pelas hipóteses $\left(g_{1}\right)$ e $\left(g_{2}\right)$, segue que, dado $\varepsilon>0$ e $q \geq \eta_{1}$, existe $C(\varepsilon, q)=C_{\varepsilon}>0$ 
tal que

$$
|g(x, s)| \leq \varepsilon|s|+C_{\varepsilon}|s|^{q} \quad \text { e } \quad|G(x, s)| \leq \varepsilon|s|^{2}+C_{\varepsilon}|s|^{q+1}
$$

para todo $s \in \mathbb{R}$. Observe que, como $\eta_{1} \in\left(3,2^{*}-1\right]$, podemos escolher $q=2^{*}-1$ nas desigualdades (4.13).

Agora, considere o nível do passo da montanha

$$
c=\inf _{\gamma \in \Gamma} \max _{t \in[0,1]} I(\gamma(t)),
$$

onde

$$
\Gamma=\left\{\gamma \in C\left([0,1], H^{1}\left(\mathbb{R}^{3}\right)\right) ; \gamma(0)=0 \text { e } I(\gamma(1))<0\right\} .
$$

Considere também o valor auxiliar

$$
c_{2}=\inf _{u \in H^{1}\left(\mathbb{R}^{N}\right) \backslash\{0\}} \max _{t \geq 0} I(t u) .
$$

O seguinte teorema, que pode ser encontrado em [18], no Capítulo 4, Teorema 6, garante a existência de uma sequência de Cerami no nível $c$ para o funcional $I$.

Teorema 35 Sejam E um espaço de Banach e $I \in C^{1}(E, \mathbb{R})$. Seja também $\Sigma$ um subconjunto fechado em $E$ que desconecta $E$ em duas componentes conexas distintas, $E_{1} e$ $E_{2}$. Suponha que $I(0)=0$ e

$\left(I_{1}\right) 0 \in E_{1}$ e existe $\alpha>0$ tal que $\left.I\right|_{\Sigma} \geq \alpha>0$;

$\left(I_{2}\right)$ existe $e \in E_{2}$ tal que $I(e)<0$.

Então, I possui uma sequência de Cerami no nivel c e $c \geq \alpha>0$.

Usando o teorema 35, podemos provar o seguinte resultado.

Lema 36 O funcional I possui uma sequência de Cerami no nivel $c>0$.

Demonstração. Vamos mostrar que $I$ satisfaz as condições do Teorema 35. Com efeito, para $\rho>0$, considere $\Sigma_{\rho}=\left\{w \in H^{1}\left(\mathbb{R}^{3}\right) ;\|f(w)\|_{H^{1}\left(\mathbb{R}^{3}\right)}=\rho\right\}$. Pela propriedade $\left(f_{1}\right), f$ é uma função contínua. Então, $\Sigma_{\rho}$ desconecta o espaço $H^{1}\left(\mathbb{R}^{3}\right)$. Mais especificamente, $\Sigma_{\rho}$ divide o espaço $H^{1}\left(\mathbb{R}^{3}\right)$ em duas componentes conectas distintas, a saber, $E_{1}=\{w \in$ $\left.H^{1}\left(\mathbb{R}^{3}\right) ;\|f(w)\|_{H^{1}\left(\mathbb{R}^{3}\right)}<\rho\right\}$ e $E_{2}=\left\{w \in H^{1}\left(\mathbb{R}^{3}\right) ;\|f(w)\|_{H^{1}\left(\mathbb{R}^{3}\right)}>\rho\right\}$. Pela desigualdade 
de Hölder e de Gagliardo-Nirenberg, temos

$$
\begin{aligned}
\int_{\mathbb{R}^{3}} V^{-}(x) f^{2}(w) d x & \leq\left\|V^{-}\right\|_{L^{3 / 2}\left(\mathbb{R}^{3}\right)}\left(\int_{\mathbb{R}^{3}}|f(w)|^{2^{*}} d x\right)^{2 / 2^{*}} \\
& \leq \frac{\left\|V^{-}\right\|_{L^{3 / 2}\left(\mathbb{R}^{3}\right)}}{S} \int_{\mathbb{R}^{3}}|\nabla f(w)|^{2} d x
\end{aligned}
$$

para todo $w \in H^{1}\left(\mathbb{R}^{3}\right)$. Como $0 \in E_{1}$ e, pela propriedade $\left(f_{2}\right)$ e pelas desigualdades (4.13) e (4.14), para $w \in \Sigma$, temos

$$
\begin{aligned}
I(w) & \geq \frac{1}{2}\left(1-\frac{\left\|V^{-}\right\|_{L^{3 / 2}\left(\mathbb{R}^{3}\right)}}{S}\right) \int_{\mathbb{R}^{3}}|\nabla f(w)|^{2} d x+\frac{1}{2} \int_{\mathbb{R}^{3}} V^{+}(x) f^{2}(x) d x \\
& -\varepsilon \int_{\mathbb{R}^{3}} f^{2}(w) d x-C_{\varepsilon} \int_{\mathbb{R}^{3}}|f(w)|^{2^{*}} d x .
\end{aligned}
$$

Pelas imersões de Sobolev, hipótese $\left(V_{3}\right)$ e Lema 33, existe uma constante $C>0$ tal que

$$
\begin{aligned}
I(w) & \geq C\|f(w)\|_{H^{1}\left(\mathbb{R}^{3}\right)}^{2}-\varepsilon\|f(w)\|_{H^{1}\left(\mathbb{R}^{3}\right)}^{2}-C_{\varepsilon}\|f(w)\|_{H^{1}\left(\mathbb{R}^{3}\right)}^{2^{*}} \\
& \geq(C-\varepsilon) \rho^{2}-C_{\varepsilon} \rho^{2^{*}} .
\end{aligned}
$$

Agora, tomando $0<\varepsilon<C$ e, em seguida, escolhendo $0<\rho<\left(\frac{C-\varepsilon}{C_{\varepsilon}}\right)^{\frac{1}{2^{*}-2}}$, segue de (4.16) que

$$
I(w) \geq(C-\varepsilon) \rho^{2}-C_{\varepsilon} \rho^{2^{*}}:=\alpha>0
$$

para todo $w \in \Sigma$. Assim, $I$ satisfaz $\left(I_{1}\right)$ do Teorema $35 \operatorname{com} \Sigma=\Sigma_{\rho}$. Para provar que $I$ também satisfaz $\left(I_{2}\right)$, pela propriedade $\left(f_{3}\right)$ e Lema 33 , existe $C>0$ tal que, se $w \neq 0$, então

$$
\begin{aligned}
I(t w) & =\frac{1}{2} \int_{\mathbb{R}^{3}}\left(|\nabla t w|^{2}+V^{+}(x) f^{2}(t w)\right) d x-\frac{1}{2} \int_{\mathbb{R}^{3}} V^{-}(x) f^{2}(t w) d x-\int_{\mathbb{R}^{3}} G(x, f(t w)) d x \\
& \leq t^{2}\left(\frac{C}{2}\|w\|_{H^{1}\left(\mathbb{R}^{3}\right)}^{2}-\int_{\mathbb{R}^{3}} \frac{G(x, f(t w))}{t^{2}} d x\right) .
\end{aligned}
$$

Logo, como provamos em (4.9),

$$
I(t w) \rightarrow-\infty \text { quando } t \rightarrow+\infty
$$

Portanto, $I(t w)<0$ para $t$ suficientemente grande e $w \neq 0$. Seja $\eta \in C_{0}^{\infty}\left(\mathbb{R}^{3}\right)$ tal que $\eta \neq 0$ e $0 \leq \eta(x)<1$ para todo $x \in \mathbb{R}^{3}$. Aplicando a propriedade $\left(f_{7}\right)$, obtemos

$$
\|f(t \eta)\|_{H^{1}\left(\mathbb{R}^{3}\right)}^{2}=t^{2} \int_{\mathbb{R}^{3}}\left|f^{\prime}(t \eta)\right|^{2}|\nabla \eta|^{2} d x+\int_{\mathbb{R}^{3}} f^{2}(t \eta) d x \geq f^{2}(t) \int_{\mathbb{R}^{3}}|\eta|^{2} d x .
$$


De $\left(f_{5}\right)$, se $t \rightarrow+\infty$, tem-se

$$
\|f(t \eta)\|_{H^{1}\left(\mathbb{R}^{3}\right)}^{2} \rightarrow+\infty
$$

Assim, podemos tomar $t$ suficientemente grande de forma que $t \eta$ pertença a $E_{2}$ e $I(t \eta)$ seja negativo. Tomando $e=t \eta$, vemos que $I$ satisfaz a condição $\left(I_{2}\right)$, o que conclui a demonstração do lema.

Observação 37 Observamos que, a fim de absorver a parte negativa $V^{-}$de $V$ nas integrais em (4.15), foi necessário trabalhar com $f(w)$ ao invés de $w$, o que implica uma restrição em tomar $q=2^{*}-1$ na desigualdade (4.13). Logo, para que o intervalo $\left(3,2^{*}-1\right]$ em $\left(g_{2}\right)$ seja não degenerado, a restrição na dimensão $N=3$ é assumida.

O seguinte resultado garante que toda sequência de Cerami para o funcional $I$ em níveis positivos é limitada. A demonstração é baseada nas ideias contidas no Lema 4.4 em [8].

Lema 38 Seja $\left(u_{n}\right) \subset H^{1}\left(\mathbb{R}^{3}\right)$ uma sequência de Cerami para I no nível $d>0$, isto é, quando $n \rightarrow \infty$

$$
I\left(u_{n}\right) \rightarrow d>0 \quad \text { e }\left\|I^{\prime}\left(u_{n}\right)\right\|\left(1+\left\|u_{n}\right\|\right) \rightarrow 0
$$

Então, a menos de subsequência, $\left(u_{n}\right)$ é limitada.

Demonstração. Primeiramente, mostraremos que a sequência $\left(f\left(u_{n}\right)\right) \subset H^{1}\left(\mathbb{R}^{3}\right)$ é limitada. Com efeito, como a sequência $\left(u_{n}\right)$ é uma sequência de Cerami para $I$ no nível $d>0$, temos que

$$
\frac{1}{2} \int_{\mathbb{R}^{3}}\left|\nabla u_{n}\right|^{2} d x+\frac{1}{2} \int_{\mathbb{R}^{3}} V(x) f^{2}\left(u_{n}\right) d x-\int_{\mathbb{R}^{3}} G\left(x, f\left(u_{n}\right)\right) d x=d+o_{n}(1) .
$$

Considere $\varphi_{n}=\frac{f\left(u_{n}\right)}{f^{\prime}\left(u_{n}\right)}$. Por $\left(f_{8}\right),\left\|\varphi_{n}\right\|_{L^{2}\left(\mathbb{R}^{3}\right)}^{2} \leq C\left\|u_{n}\right\|^{2}$ para algum $C>0$. Também vale

$$
\left|\nabla \varphi_{n}\right|=\left(1+\frac{2 f^{2}\left(u_{n}\right)}{1+2 f^{2}\left(u_{n}\right)}\right)\left|\nabla u_{n}\right| \leq 2\left|\nabla u_{n}\right| .
$$

Assim, $\varphi_{n} \in H^{1}\left(\mathbb{R}^{3}\right)$ com $\left\|\varphi_{n}\right\|_{H^{1}\left(\mathbb{R}^{3}\right)} \leq C\left\|u_{n}\right\|_{H^{1}\left(\mathbb{R}^{3}\right)}$. Lembrando que $\left(u_{n}\right)$ é uma sequência de Cerami, segue que

$$
\begin{aligned}
o_{n}(1)=I^{\prime}\left(u_{n}\right) \varphi_{n}= & \int_{\mathbb{R}^{3}}\left(1+\frac{2 f^{2}\left(u_{n}\right)}{1+2 f^{2}\left(u_{n}\right)}\right)\left|\nabla u_{n}\right|^{2} d x \\
& +\int_{\mathbb{R}^{3}} V(x) f^{2}\left(u_{n}\right) d x-\int_{\mathbb{R}^{3}} g\left(x, f\left(u_{n}\right)\right) f\left(u_{n}\right) d x .
\end{aligned}
$$


Calculando (4.18) - $\frac{1}{4}(4.19)$, obtemos

$$
\begin{aligned}
d+o_{n}(1) & =\int_{\mathbb{R}^{3}}\left(\frac{1}{2}-\frac{1}{4}\left(1+\frac{2 f^{2}\left(u_{n}\right)}{1+2 f^{2}\left(u_{n}\right)}\right)\right)\left|\nabla u_{n}\right|^{2} d x+\frac{1}{4} \int_{\mathbb{R}^{3}} V(x) f^{2}\left(u_{n}\right) d x \\
& +\int_{\mathbb{R}^{3}}\left(\frac{g\left(x, f\left(u_{n}\right)\right) f\left(u_{n}\right)}{4}-G\left(x, f\left(u_{n}\right)\right)\right) d x
\end{aligned}
$$

Pela hipótese $\left(g_{3}\right)$ e como $\mu>4$, a última integral em (4.20) é não negativa. Logo,

$$
\int_{\mathbb{R}^{3}}\left(\frac{1}{2}-\frac{1}{4}\left(1+\frac{2 f^{2}\left(u_{n}\right)}{1+2 f^{2}\left(u_{n}\right)}\right)\right)\left|\nabla u_{n}\right|^{2} d x+\frac{1}{4} \int_{\mathbb{R}^{3}} V(x) f^{2}\left(u_{n}\right) d x \leq d+o_{n}(1),
$$

ou equivalentemente,

$$
\frac{1}{4} \int_{\mathbb{R}^{3}} \frac{\left|\nabla u_{n}\right|^{2}}{1+2 f^{2}\left(u_{n}\right)} d x+\frac{1}{4} \int_{\mathbb{R}^{3}} V(x) f^{2}\left(u_{n}\right) d x \leq d+o_{n}(1)
$$

Como $\nabla f\left(u_{n}\right)=f^{\prime}\left(u_{n}\right) \nabla u_{n}$ e $\left(f^{\prime}\left(u_{n}\right)\right)^{2}=\frac{1}{1+2 f^{2}\left(u_{n}\right)}$, segue de (4.21) que

$$
\frac{1}{4} \int_{\mathbb{R}^{3}}\left|\nabla f\left(u_{n}\right)\right|^{2} d x+\frac{1}{4} \int_{\mathbb{R}^{3}} V(x) f^{2}\left(u_{n}\right) d x \leq d+o_{n}(1)
$$

Por (4.14), tem-se

$$
\frac{1}{4}\left[\left(1-\frac{\left\|V^{-}\right\|_{L^{3 / 2}\left(\mathbb{R}^{3}\right)}}{S}\right) \int_{\mathbb{R}^{3}}\left|\nabla f\left(u_{n}\right)\right|^{2} d x+\int_{\mathbb{R}^{3}} V^{+}(x) f^{2}\left(u_{n}\right) d x\right] \leq d+o_{n}(1) .
$$

Portanto, a hipótese $\left(V_{3}\right)$ e o Lema 33 garantem que $\left(f\left(u_{n}\right)\right)$ é limitada. Por isso, pelas imersões de Sobolev e pela desigualdade (4.13) $\operatorname{com} \varepsilon=1$, existe $C>0$ tal que

$$
\int_{\mathbb{R}^{3}} G\left(x, f\left(u_{n}\right)\right) d x \leq \int_{\mathbb{R}^{3}} f^{2}\left(u_{n}\right) d x+C_{\varepsilon} \int_{\mathbb{R}^{N}}\left|f\left(u_{n}\right)\right|^{2^{*}} d x \leq C .
$$

Aplicando (4.22) em (4.18), temos pela desigualdade de Hölder e pelas imersões de Sobolev que

$$
\begin{aligned}
\frac{1}{2} \int_{\mathbb{R}^{3}}\left|\nabla u_{n}\right|^{2} d x & \leq d+o_{n}(1)+C+\frac{\left\|V^{-}\right\|_{L^{3 / 2}\left(\mathbb{R}^{3}\right)}}{2}\left(\int_{\mathbb{R}^{3}}\left|f\left(u_{n}\right)\right|^{2^{*}} d x\right)^{2 / 2^{*}} \\
& \leq d+o_{n}(1)+C+\frac{C\left\|V^{-}\right\|_{L^{3 / 2}\left(\mathbb{R}^{3}\right)}}{2}\left\|f\left(u_{n}\right)\right\|_{H^{1}\left(\mathbb{R}^{3}\right)}^{2}
\end{aligned}
$$


concluindo que $\int_{\mathbb{R}^{3}}\left|\nabla u_{n}\right|^{2} d x$ é uma sequência real limitada. Agora, a fim de provar que $\left(u_{n}\right)$ é limitada, note que, pela propriedade $\left(f_{7}\right)$, temos

$$
\int_{\left|u_{n}\right| \leq 1}\left|u_{n}\right|^{2} d x \leq \frac{1}{f^{2}(1)} \int_{\left|u_{n}\right| \leq 1} f^{2}\left(u_{n}\right) d x \leq \frac{1}{f^{2}(1)} \int_{\mathbb{R}^{3}} f^{2}\left(u_{n}\right) d x \leq C
$$

Por outro lado, pela propriedade $\left(f_{5}\right)$ e pelas imersões contínuas de Sobolev, existe $C>0$ tal que

$$
\int_{\left|u_{n}\right| \geq 1}\left|u_{n}\right|^{2} d x \leq \frac{1}{C} \int_{\left|u_{n}\right| \geq 1}\left|f\left(u_{n}\right)\right|^{4} d x \leq C\left\|f\left(u_{n}\right)\right\|_{H^{1}\left(\mathbb{R}^{3}\right)}^{4} \leq C
$$

uma vez que $\left(f\left(u_{n}\right)\right)$ é limitada em $H^{1}\left(\mathbb{R}^{3}\right)$. De (4.23), (4.24) e (4.25), concluímos que $\left(u_{n}\right)$ é limitada em $H^{1}\left(\mathbb{R}^{3}\right)$ e o lema está provado.

Recordamos aqui um resultado de existência de uma solução de energia mínima para o problema limite $\left(P_{\infty}\right)$.

Teorema 39 Suponha que $\left(g_{1}\right)-\left(g_{2}\right)$ sejam válidas. Então, o problema $\left(P_{\infty}\right)$ possui uma solução positiva de energia mínima $v_{0} \in H^{1}\left(\mathbb{R}^{3}\right)$ tal que

$$
I_{\infty}\left(v_{0}\right)=m_{\infty}:=\inf \left\{I_{\infty}(w) ; w \neq 0 \quad \text { e } \quad I_{\infty}^{\prime}(w)=0\right\}>0 .
$$

A demonstração deste teorema é baseada nos resultados contidos em [4]. Por questão de completude, vamos incluí-la aqui.

Demonstração. Vamos verificar que a função

$$
k(s):=f^{\prime}(s)\left(h(f(s))-V_{\infty} f(s)\right), \quad s \in \mathbb{R},
$$

satisfaz as condições apresentadas em [4], no Teorema 1. Com efeito, temos que $k$ é contínua e, como $h(0)=f(0)=0$, tem-se $k(0)=0$. Além disso, em vista que $f^{\prime}(0)=1$, por $\left(g_{1}\right)$, obtemos

$$
k^{\prime}(0)=f^{\prime \prime}(0)\left(h(f(0))-V_{\infty} f(0)\right)+f^{\prime}(0)\left(h^{\prime}(f(0)) f^{\prime}(0)-V_{\infty} f^{\prime}(0)\right)=-V_{\infty}<0 .
$$

Segue da hipótese $\left(g_{2}\right)$ e da propriedade $\left(f_{9}\right)$ que

$$
\begin{aligned}
0 \leq \frac{|k(s)|}{|s|^{2^{*}-1}} \leq \frac{\left|f^{\prime}(s) h(f(s))\right|}{|s|^{2^{*}-1}}+\frac{V_{\infty}\left|f^{\prime}(s) f(s)\right|}{|s|^{2^{*}-1}} & \leq \frac{|h(f(s))||f(s)|^{\eta_{1}}}{\left.|f(s)|\right|_{1}} \frac{C}{|s|^{2^{*}-1}}+\frac{C}{|s|^{2^{*}-1}} \\
& \leq C \frac{|h(f(s))|}{\left.|f(s)|\right|^{\eta_{1}}} \frac{|s|^{\frac{\eta_{1}}{2}}}{|s|^{2^{*}-1}}+\frac{C}{|s|^{2^{*}-1}} \\
& =o_{s}(1)
\end{aligned}
$$


quando $s \rightarrow+\infty$. Isto mostra que $k(s) \rightarrow 0$ quando $s \rightarrow+\infty$. Por fim, precisamos mostrar que existe $\xi_{0}>0$ tal que $K\left(\xi_{0}\right):=\int_{0}^{\xi_{0}} k(t) d t=H\left(f\left(\xi_{0}\right)\right)-\frac{V_{\infty}}{2} f^{2}\left(\xi_{0}\right)=$ $f^{2}\left(\xi_{0}\right)\left(\frac{H\left(f\left(\xi_{0}\right)\right)}{f^{2}\left(\xi_{0}\right)}-\frac{V_{\infty}}{2}\right)>0$. Mas isto segue diretamente da hipótese $\left(g_{2}\right)$, do fato de $f(s) \rightarrow+\infty$ quando $s \rightarrow+\infty$, da propriedade $\left(f_{3}\right)$ e de (4.9). Segue o resultado.

Com o intuito de provar a compacidade, é importante considerar alguma comparação entre os funcionais $I$ e $I_{\infty}$.

Lema $40 c \leq c_{2}<m_{\infty}$.

Demonstração. Seja $u \in H^{1}\left(\mathbb{R}^{3}\right) \backslash\{0\}$. Como na prova do Lema 34, existe $t_{0}>0$ tal que $I(t u)<0$ se $t \geq t_{0}$ para algum $t_{0}>0$. Considere $\gamma_{u} \in C\left([0,1], H^{1}\left(\mathbb{R}^{3}\right)\right)$ dado por $\gamma_{u}(s)=s t_{0} u$. Temos que

$$
\inf _{u \in H^{1}\left(\mathbb{R}^{3}\right) \backslash\{0\}} \max _{t \geq 0} I(t u)=\inf _{u \in H^{1}\left(\mathbb{R}^{3}\right) \backslash\{0\}} \max _{s \in[0,1]} I\left(\gamma_{u}(s)\right)
$$

Desde que $\gamma_{u}(0)=0$ e $I\left(\gamma_{u}(1)\right)<0$, vale que $\gamma_{u} \in \Gamma$ e, então,

$$
c_{2}=\inf _{u \in H^{1}\left(\mathbb{R}^{3}\right) \backslash\{0\}} \max _{t \geq 0} I(t u)=\inf _{u \in H^{1}\left(\mathbb{R}^{3}\right) \backslash\{0\}} \max _{s \in[0,1]} I\left(\gamma_{u}(s)\right) \geq \inf _{\gamma \in \Gamma} \max _{t \in[0,1]} I(\gamma(t))=c .
$$

Isto mostra que $c \leq c_{2}$. Pelo Lema 34 , para $w \in \mathcal{N}_{\infty}$, temos $w \neq 0$ e, da hipótese $\left(V_{2}\right)$, segue que

$$
c_{2} \leq \max _{t \geq 0} I(t w)=I\left(s_{w} w\right)<I_{\infty}\left(s_{w} w\right) \leq \max _{t \geq 0} I_{\infty}(t w)=I_{\infty}\left(t_{w} w\right)
$$

Agora, do Teorema 39, escolhemos $v_{0} \in H^{1}\left(\mathbb{R}^{3}\right)$ e observamos que, de (4.26),

$$
c<I_{\infty}\left(t_{v_{0}} v_{0}\right)
$$

Como $v_{0}>0$ e $I_{\infty}^{\prime}\left(v_{0}\right) v_{0}=0$, segue do Lema 34 que $t_{v_{0}}=1$. Assim, de (4.27), $c<m_{\infty}$ e o lema está provado.

O lema seguinte é um resultado padrão.

Lema 41 (Splitting) Seja $\left(u_{n}\right)$ uma sequência limitada em $H^{1}\left(\mathbb{R}^{3}\right)$ tal que

$$
I\left(u_{n}\right) \rightarrow d>0 \quad \text { e } \quad\left\|I^{\prime}\left(u_{n}\right)\right\|\left(1+\left\|u_{n}\right\|\right) \rightarrow 0
$$

Então, a menos de subsequência, existe uma solução $u_{0}$ de $(P)$, um número $k \in \mathbb{N} \cup\{0\}$, $k$ funções $u_{1}, \cdots, u_{k}$ e $k$ sequências de pontos $\left(y_{n}^{j}\right) \in \mathbb{R}^{3}, 1 \leq j \leq k$, satisfazendo: 
a) $u_{n} \rightarrow u_{0}$ em $H^{1}\left(\mathbb{R}^{3}\right)$ ou

b) $u_{j}$ são soluções não triviais of $\left(P_{\infty}\right)$;

c) $\left|y_{n}^{j}\right| \rightarrow+\infty e\left|y_{n}^{j}-y_{n}^{i}\right| \rightarrow+\infty i \neq j$;

d) $I\left(u_{n}\right) \rightarrow I\left(u_{0}\right)+\sum_{i=1}^{k} I_{\infty}\left(u_{j}\right)$.

Observação 42 Os argumentos em [10] e [50] podem ser aplicados para se provar o Lema 41. Tal lema é uma versão da concentração e compacidade de P. L. Lions [30] e pode ser encontrada em [43]. Os principais ingredientes são o Lema de Lions e o Lema de Brezis-Lieb [5]. A demonstração pode ser adaptada de [10], Proposição 2.31 (veja 2.11 e 2.46 a 2.55 lá). Sob a condição $\left(g_{5}\right)$, desigualdades (4.13), pela regularidade das funções $g, G$ e $f$ e propriedade $\left(f_{2}\right)$, o teorema do valor médio garante um limite uniforme em $\mathbb{R}^{N} \backslash \Omega$ com $\Omega \subset \subset \mathbb{R}^{N}$ escolhido apropriadamente. No conjunto $\Omega$, o argumento depende da imersão compacta de Sobolev $H^{1}\left(\mathbb{R}^{3}\right) \hookrightarrow L_{\text {loc }}^{q+1}\left(\mathbb{R}^{3}\right)$, onde $2 \leq q+1<2^{*}$. Neste sentido, (4.13) e a propriedade $\left(f_{10}\right)$ implicam que, para qualquer $q \in\left[\eta_{1}, 2^{*}-1\right]$, vale $q u e|f(s)|^{q+1} \leq 2^{\frac{1}{4}}|s|^{\frac{q+1}{2}}$ com $\frac{q+1}{2}<2^{*}$, concluindo a demonstração do lema.

Finalmente, apresentamos a prova do resultado principal deste capítulo.

Prova do Teorema 31: Pelo Lema 36, existe uma sequência de Cerami $\left(u_{n}\right)$ para o funcional $I$ no nível $c>0$. O Lema 38, por sua vez, garante que, a menos de subsequência, $\left(u_{n}\right)$ é limitada e, então, $u_{n} \rightarrow u_{0}$ em $H^{1}\left(\mathbb{R}^{3}\right)$ para algum $u_{0} \in H^{1}\left(\mathbb{R}^{3}\right)$. Se $u_{n} \rightarrow u_{0}$ não fosse verdade, o Lema 41 nos forneceria $k$ soluções $u_{i} \in H^{1}\left(\mathbb{R}^{3}\right)$ do problema $\left(P_{\infty}\right)$ tais que

$$
I\left(u_{n}\right) \rightarrow I\left(u_{0}\right)+\sum_{i=1}^{k} I_{\infty}\left(u_{i}\right) \geq k m_{\infty} \geq m_{\infty},
$$

contradizendo o Lema 40, uma vez que $I\left(u_{n}\right)=c+o_{n}(1)$. Aqui, usamos que $I\left(u_{0}\right) \geq 0$. Este fato é verdadeiro, pois, como $u_{n} \rightarrow u_{0}$, pelas imersões compactas de Sobolev, temos $I^{\prime}\left(u_{0}\right)=0$. Logo, se $\mu_{1}:=\mu / 2>2$, então

$$
\begin{aligned}
I\left(u_{0}\right)=I\left(u_{0}\right)-\frac{1}{\mu_{1}} I^{\prime}\left(u_{0}\right) u_{0}= & \left(\frac{1}{2}-\frac{1}{\mu_{1}}\right) \int_{\mathbb{R}^{3}}\left|\nabla u_{0}\right|^{2} d x \\
& +\int_{\mathbb{R}^{3}} V^{+}(x)\left(\frac{f^{2}\left(u_{0}\right)}{2}-\frac{f\left(u_{0}\right) f^{\prime}\left(u_{0}\right) u_{0}}{\mu_{1}}\right) d x \\
& +\int_{\mathbb{R}^{3}} V^{-}(x)\left(\frac{f\left(u_{0}\right) f^{\prime}\left(u_{0}\right) u_{0}}{\mu_{1}}-\frac{f^{2}\left(u_{0}\right)}{2}\right) d x \\
& +\int_{\mathbb{R}^{3}}\left(\frac{g\left(x, f\left(u_{0}\right)\right) f^{\prime}\left(u_{0}\right) u_{0}}{\mu_{1}}-G\left(x, f\left(u_{0}\right)\right)\right) d x
\end{aligned}
$$


que se torna pelas propriedades $\left(f_{2}\right)$ e $\left(f_{6}\right)$ em

$$
\begin{aligned}
I\left(u_{0}\right) & \geq\left(\frac{1}{2}-\frac{1}{\mu_{1}}\right)\left[\int_{\mathbb{R}^{3}}\left|\nabla f\left(u_{0}\right)\right|^{2} d x+\int_{\mathbb{R}^{3}} V^{+}(x) f^{2}\left(u_{0}\right)\right] d x \\
& -\left(\frac{1}{2}-\frac{1}{2 \mu_{1}}\right) \int_{\mathbb{R}^{3}} V^{-}(x) f^{2}\left(u_{0}\right) d x \\
& +\int_{\mathbb{R}^{3}}\left(\frac{g\left(x, f\left(u_{0}\right)\right)}{\mu_{1} f\left(u_{0}\right)} f\left(u_{0}\right) f^{\prime}\left(u_{0}\right) u_{0}-G\left(x, f\left(u_{0}\right)\right)\right) d x
\end{aligned}
$$

Pela propriedade $\left(g_{3}\right), \frac{g(x, s)}{s}>0$ para todo $s \neq 0$. Portanto, segue da propriedade $\left(f_{6}\right)$ que

$$
\begin{aligned}
I\left(u_{0}\right) & \geq\left(\frac{1}{2}-\frac{1}{\mu_{1}}\right)\left[\int_{\mathbb{R}^{3}}\left|\nabla f\left(u_{0}\right)\right|^{2} d x+\int_{\mathbb{R}^{3}} V^{+}(x) f^{2}\left(u_{0}\right)\right] d x \\
& -\left(\frac{1}{2}-\frac{1}{2 \mu_{1}}\right) \int_{\mathbb{R}^{3}} V^{-}(x) f^{2}\left(u_{0}\right) d x \\
& +\int_{\mathbb{R}^{3}}\left(\frac{g\left(x, f\left(u_{0}\right)\right) f\left(u_{0}\right)}{2 \mu_{1}}-G\left(x, f\left(u_{0}\right)\right)\right) d x .
\end{aligned}
$$

Por (4.14) e pela definição da constantes $S$ e $\mu_{1}$, temos

$$
\begin{aligned}
I\left(u_{0}\right) & \geq\left(\frac{1}{2}-\frac{1}{\mu_{1}}\right)\left[\int_{\mathbb{R}^{3}}\left|\nabla f\left(u_{0}\right)\right|^{2} d x+\int_{\mathbb{R}^{3}} V^{+}(x) f^{2}\left(u_{0}\right)\right] d x \\
& -\left(\frac{1}{2}-\frac{1}{2 \mu_{1}}\right) \frac{\left\|V^{-}\right\|_{L^{3 / 2}\left(\mathbb{R}^{3}\right)}}{S} \int_{\mathbb{R}^{3}}\left|\nabla f\left(u_{0}\right)\right|^{2} d x \\
& +\int_{\mathbb{R}^{3}}\left(\frac{g\left(x, f\left(u_{0}\right)\right) f\left(u_{0}\right)}{\mu}-G\left(x, f\left(u_{0}\right)\right)\right) d x .
\end{aligned}
$$

A hipótese $\left(V_{3}\right)$ garante que

$$
\left(\frac{1}{2}-\frac{1}{\mu_{1}}\right)-\left(\frac{1}{2}-\frac{1}{2 \mu_{1}}\right) \frac{\left\|V^{-}\right\|_{L^{3 / 2}\left(\mathbb{R}^{3}\right)}}{S}>0
$$

Assim, por $\left(g_{3}\right)$ e pelo Lema 33 , existe uma constante $C>0$ tal que

$$
I\left(u_{0}\right) \geq C\left\|f\left(u_{0}\right)\right\|_{H^{1}\left(\mathbb{R}^{3}\right)}^{2} \geq 0 .
$$

Como $I\left(u_{n}\right)=c+o_{n}(1)$ e $c>0, u_{0}$ é uma solução fraca não trivial de $(P)$. Isto conclui a prova do teorema. 


\section{Capítulo 5}

\section{Apêndice A}

Seja $\partial B_{1}$ a fronteira do conjunto $B_{1}$, onde $B_{1}$ é a bola aberta de centro na origem e raio 1 contida no espaço de dimensão finita gerado pelas funções $u_{0}^{+}(\cdot-y), \phi_{1}, \cdots, \phi_{k}$.

Lema 43 Vale que

$$
\lim _{R \rightarrow \infty} \int_{\mathbb{R}^{N}}\left(\frac{m}{2}-\frac{F(R u)}{(R u)^{2}}\right) u^{2} d x=0
$$

uniformemente para $u \in \partial B_{1}$.

Demonstração. De fato, para cada $R=n \in \mathbb{N}$, considere $J_{n}: \partial B_{1} \rightarrow \mathbb{R}$ a função dada por $J_{n}(u)=\int_{\mathbb{R}^{N}}\left(\frac{m}{2}-\frac{F(n u)}{(n u)^{2}}\right) u^{2} d x$. A continuidade da função $F$ mostra que $J_{n}$ é uma função contínua para cada $n$. A hipótese $\left(f_{2}\right)$ e a equivalência das normas $\|\cdot\|$ e $\|\cdot\|_{E}$ nos fornecem

$$
0 \leq J_{n}(u)=\int_{\mathbb{R}^{N}}\left(\frac{m}{2}-\frac{F(n u)}{(n u)^{2}}\right) u^{2} d x \leq m\|u\|_{H^{1}\left(\mathbb{R}^{N}\right)}^{2} \leq C
$$

para qualquer $u \in \partial B_{1}$. Assim, a continuidade do funcional $J_{n}$ no conjunto compacto $\partial B_{1}$ garante que, para cada $n$, a função $J_{n}$ assume um valor máximo em, digamos, $u_{n} \in \partial B_{1}$. Considere $\left(u_{n}\right)$ a sequência destes pontos de máximo. Como $\left\|u_{n}\right\|=1$ para cada $n$ e o espaço gerado pelas funções $u_{0}^{+}(\cdot-y), \phi_{1}, \cdots, \phi_{k}$ é de dimensão finita, existe $\bar{u} \in \partial B_{1}$ tal que, a menos de subsequência,

$$
u_{n} \rightarrow \bar{u}
$$

fortemente na norma $\|\cdot\|$. Para toda $u \in \partial B_{1}$ e para cada $n$,

$$
0 \leq J_{n}(u) \leq J_{n}\left(u_{n}\right)
$$


isto é,

$$
0 \leq \int_{\mathbb{R}^{N}}\left(\frac{m}{2}-\frac{F(n u)}{(n u)^{2}}\right) u^{2} d x \leq \int_{\mathbb{R}^{N}}\left(\frac{m}{2}-\frac{F\left(n u_{n}\right)}{\left(n u_{n}\right)^{2}}\right) u_{n}^{2} d x
$$

para toda $u$ e para cada $n$. Antes de passar o limite $n \rightarrow \infty$, note que

$$
u_{n}(x) \rightarrow \bar{u}(x) \quad \text { q.t.p. em } \quad \mathbb{R}^{N}
$$

Assim, se $\bar{u}(x) \neq 0$, segue que $\left|n u_{n}(x)\right| \rightarrow \infty$ quando $n \rightarrow \infty$. Portanto, a hipótese $\left(f_{2}\right)$ garante que

$$
\left(\frac{m}{2}-\frac{F\left(n u_{n}(x)\right)}{\left(n u_{n}(x)\right)^{2}}\right) u_{n}^{2}(x) \rightarrow 0
$$

quando $n \rightarrow \infty$. Se $\bar{u}(x)=0$, também temos (5.3). Pela convergência forte (5.1), existe uma função $\bar{h} \in L^{1}\left(\mathbb{R}^{N}\right)$ tal que, a menos de subsequência,

$$
0 \leq\left(\frac{m}{2}-\frac{F\left(n u_{n}(x)\right)}{\left(n u_{n}(x)\right)^{2}}\right) u_{n}^{2}(x) \leq m u_{n}^{2}(x) \leq m \bar{h}(x) \in L^{1}\left(\mathbb{R}^{N}\right) \text {. }
$$

Por (5.3) e (5.4), o Teorema da Convergência Dominada de Lebesgue garante que

$$
\lim _{n \rightarrow \infty} \int_{\mathbb{R}^{N}}\left(\frac{m}{2}-\frac{F\left(n u_{n}\right)}{\left(n u_{n}\right)^{2}}\right) u_{n}^{2} d x=0 .
$$

Logo, fazendo $n \rightarrow \infty$ em (5.2), obtemos

$$
\lim _{n \rightarrow \infty} \int_{\mathbb{R}^{N}}\left(\frac{m}{2}-\frac{F(n u)}{(n u)^{2}}\right) u^{2} d x=0
$$

uniformemente em $u \in \partial B_{1}$, como queríamos demonstrar.

Sob as mesmas notações do Lema 43, o seguinte resultado é válido.

Lema 44 O limite

$$
\lim _{R \rightarrow \infty} \int_{\mathbb{R}^{N}}\left(\frac{a(x)}{2}-\frac{F(x, R u)}{(R u)^{2}}\right) u^{2} d x=0
$$

uniformemente para $u \in \partial B_{1}$.

Demonstração. Com efeito, para cada $R=n \in \mathbb{N}$, considere $J_{n}: \partial B_{1} \rightarrow \mathbb{R}$ a função dada por $J_{n}(u)=\int_{\mathbb{R}^{N}}\left(\frac{a(x)}{2}-\frac{F(x, n u)}{(n u)^{2}}\right) u^{2} d x$. A continuidade da função $F$ mostra que $J_{n}$ é uma função contínua para cada $n$. A hipótese $\left(f_{2}\right)$ e a equivalência das normas $\|\cdot\| \mathrm{e}\|\cdot\|_{E}$ nos fornecem

$$
0 \leq J_{n}(u)=\int_{\mathbb{R}^{N}}\left(\frac{a(x)}{2}-\frac{F(x, n u)}{(n u)^{2}}\right) u^{2} d x \leq a_{0}\|u\|_{H^{1}\left(\mathbb{R}^{N}\right)}^{2} \leq C
$$


para toda $u \in \partial B_{1}$, onde $a_{0}=\sup _{x \in \mathbb{R}^{N}} a(x)$. Assim, a continuidade do funcional $J_{n}$ no conjunto compacto $\partial B_{1}$ garante que, para cada $n$, a função $J_{n}$ assume um valor máximo em, digamos, $u_{n} \in \partial B_{1}$. Considere $\left(u_{n}\right)$ a sequência destes pontos de máximo. Como $\left\|u_{n}\right\|=1$ para cada $n$ e o espaço gerado pelas funções $u_{0}^{+}(\cdot-y), \phi_{1}, \cdots, \phi_{k}$ é de dimensão finita, existe $\bar{u} \in \partial B_{1}$ tal que, a menos de subsequência,

$$
u_{n} \rightarrow \bar{u}
$$

fortemente na norma $\|\cdot\|$. Para toda $u \in \partial B_{1}$ e para cada $n$,

$$
0 \leq J_{n}(u) \leq J_{n}\left(u_{n}\right)
$$

isto é,

$$
0 \leq \int_{\mathbb{R}^{N}}\left(\frac{a(x)}{2}-\frac{F(x, n u)}{(n u)^{2}}\right) u^{2} d x \leq \int_{\mathbb{R}^{N}}\left(\frac{a(x)}{2}-\frac{F\left(x, n u_{n}\right)}{\left(n u_{n}\right)^{2}}\right) u_{n}^{2} d x
$$

para qualquer $u \in \partial B_{1}$ e para cada $n$. Antes de passar o limite $n \rightarrow \infty$, note que

$$
u_{n}(x) \rightarrow \bar{u}(x) \quad \text { q.t.p. em } \quad \mathbb{R}^{N}
$$

Assim, se $\bar{u}(x) \neq 0$, segue que $\left|n u_{n}(x)\right| \rightarrow \infty$ quando $n \rightarrow \infty$. Portanto, a hipótese $\left(f_{2}\right)$ garante que

$$
\left(\frac{a(x)}{2}-\frac{F\left(x, n u_{n}(x)\right)}{\left(n u_{n}(x)\right)^{2}}\right) u_{n}^{2}(x) \rightarrow 0
$$

quando $n \rightarrow \infty$. Se $\bar{u}(x)=0$, também temos (5.7). Pela convergência forte (5.5), existe uma função $\bar{h} \in L^{1}\left(\mathbb{R}^{N}\right)$ tal que, a menos de subsequência,

$$
0 \leq\left(\frac{a(x)}{2}-\frac{F\left(x, n u_{n}(x)\right)}{\left(n u_{n}(x)\right)^{2}}\right) u_{n}^{2}(x) \leq a_{0} u_{n}^{2}(x) \leq a_{0} \bar{h}(x) \in L^{1}\left(\mathbb{R}^{N}\right) .
$$

Por (5.7) e (5.8), o Teorema da Convergência Dominada de Lebesgue garante que

$$
\lim _{n \rightarrow \infty} \int_{\mathbb{R}^{N}}\left(\frac{a(x)}{2}-\frac{F\left(x, n u_{n}\right)}{\left(n u_{n}\right)^{2}}\right) u_{n}^{2} d x=0 .
$$

Logo, fazendo $n \rightarrow \infty$ em (5.6), obtemos

$$
\lim _{n \rightarrow \infty} \int_{\mathbb{R}^{N}}\left(\frac{a(x)}{2}-\frac{F(x, n u)}{(n u)^{2}}\right) u^{2} d x=0
$$

uniformemente em $u \in \partial B_{1}$, como queríamos demonstrar. 


\section{Referências Bibliográficas}

[1] J. F.L. Aires e M. A. S. Souto, Existence of solutions for a quasilinear Schrödinger equation with vanishing potentials. J. Math. Anal. Appl., 416 (2014), 924-946.

[2] N. Arckemman, On a periodic Schrödinger equation with nonlocal superlinear part, Mathematische Zeitschrift, 248 (2004), 423-443.

[3] A. Azzollini e A. Pomponio, On the Schrödinger equation in $\mathbb{R}^{N}$ under the effect of a general nonlinear term, Indiana University Mathematics Journal, 58 (2009), 3.

[4] H. Berestycki e P. L. Lions, Nonlinear scalar field equations. I. Existence of a ground state, Arch. Rational Mech. Anal., 82 (1983), 313-345.

[5] H. Brezis e E. Lieb, A relation between pointwise convergence of functions and convergence of functionals, Proc. Amer. Math. Soc., 88 (1983), 486-490.

[6] G. Cerami, Un criterio di ezistenza per i punti critici su varietà illimitate, Rend. Accad. Sc. Lett. Inst. Lombardo, 112 (1978), 332-336.

[7] M. Clapp e L. A. Maia, A positive bound state for an asymptotically linear or superlinear Schrödinger equation. J. Differential Equations. Preprint (2015).

[8] M. Colin e L. Jeanjean, Solutions for a quasilinear Schrödinger equation: a dual approach. Nonlinear Analysis, 56 (2004), 213-226.

[9] D. G. Costa e H. Tehrani, Existence and multiplicity results for a class of Schrödinger equations with indefinite nonlinearities, Adv. Difference Equation, 8 (2003), 13191340 .

[10] V. Coti-Zelati e P. Rabinowitz, Homoclinic type solutions for a semilinear elliptic PDE on $\mathbb{R}^{N}$, Comm. Pure Appl. Math, 46 (1992), 1217-1269.

[11] R. Dautray e J-L. Lions, Mathematical analysis and numerical methods for science and technology, Volume 1, Physical origins and classical methods, Springer-Verlag, Berlin, 1990. 
[12] Y. Ding, Variational methods for strongly indefinite problems, Volume 7, World Scientific, Chine, 2007.

[13] Y. Ding e C. Lee, Multiple solutions of Schrödinger equations with indefinite linear part and super or asymptotically linear terms, J. Differential Equations, 222 (2006), 137-163.

[14] Y. Ding e B. Ruf, Solutions of a nonlinear Dirac equation with external fields, Arch. Rational Mech. Anal., 190 (2008), 57-82.

[15] J. M. do ó e U. Severo, Solitary waves for a class of quasilinear Schrödinger equations in dimension two. Calc. Var., 38 (2010), 275-315.

[16] A. L. Edelson e C. A. Stuart, The Principle Branch of Solutions of a Nonlinear Elliptic Eigenvalue Problem on $\mathbb{R}^{N}$. J. Differential Equations, 124 (1996), 279-301.

[17] Y. Egorov e V. Kondratiev, On Spectral Theorey of Elliptic Operators, Birkhäuser, Basel, 1996.

[18] I. Ekeland, Convexity Methods in Hamiltonian Mechanics, Springer-Velarg, Berlin, 1990.

[19] G. Évéquoz e T. Weth, Entire solutions to nonlinear scalar field equations with indefinite linear part, Adv. Nonlinear Stud., 12 (2012), 281-314.

[20] X-D. Fang e Z-Q. Han, Existence of nontrivial solutions for a quasilinear Schrödinger equations with sign-changing potential. Elect. J. of Differ. Equations., 5 (2014), 1-8.

[21] X. D. Fang e A. Szulkin, Multiple solutions for a quasilinear Schrödinger equation. J. Differential Equations, 254 (2013), 2015-2032.

[22] M. F. Furtado, L. A. Maia e E. S. Medeiros, Positive and nodal solitons for a nonlinear Schrödinger equation with indefinite potential, Adv. Nonlinear Stud., 8 (2008), 353-373.

[23] D. Gilbarg e N. S. Trundinger, Elliptic Partial Differential Equations of Second Order, Spring.

[24] L. Jeanjean, On the existence of bounded Palais-Smale sequences and application to a Landesman-Lazer-type problem set on $\mathbb{R}^{N}$, Proc. Roy. Soc. Edinburgh Sect. A, 129 (1999), 787-809. 
[25] L. Jeanjean e K. Tanaka, A positive solution for an asymptotically linear elliptic problem on $\mathbb{R}^{N}$ autonomous at infinity, ESAIM Control Optim. Calc. Var., 7 (2002), 597-614.

[26] L. Jeanjean e K. Tanaka, A remark on least energy solutions in $\mathbb{R}^{N}$, Proc. Amer. Math. Soc., 131 (2002), 2399-2408.

[27] W. Kryszewski e A. Szulkin, Generalized linking theorem with an application to semilinear Schrödinger equations, Adv. Diff. Equas., 3 (1998), 441-472.

[28] G. Li e A. Szulkin, An asymptotically periodic Schrödinger equation with indefinite linear part, Communications in Contemporary Mathematics, 4 (2002), 763-776.

[29] G. Li e C. Wang, The existence of a nontrivial solution to a nonlinear elliptic problem of linking type without the Ambrosetti-Rabinowitz condition, Annales Academiae Scientiarum Fennicae, 36 (2011), 461-480.

[30] P.L. Lions, The concentration-compactness principle in the calculus of variations. The locally compact case. Parts I and II, Ann. Inst. H. Poincaré Anal. Non Linéaire, 1 (1984), 109-145 and 223-283.

[31] J. Liu, Y. Wang e Z. Wang, Soliton solutions for quasilinear Schrödinger equations II. J. Differential Equations, 187 (2003), 473-493.

[32] Z. Liu, J. Su e T. Weth, Compactness results for Schrödinger equations with asymptotically linear terms, Journal of Differential Equations, 231 (2006), 501-512.

[33] A. Moameni, Existence of soliton solutions for a quasilinear Schrödinger equation involving critical exponent in $\mathbb{R}^{N}$. J. Differential Equations, 229 (2006), 570-587.

[34] R.S. Palais, The principle of symmetric criticality, Comm. Math. Phys., 69 (1979), $19-30$.

[35] A. A. Pankov, Periodic nonlinear Schrödinger equation with application to photonic crystals, Milan J. Math., 73 (2005), 259-287.

[36] A. A. Pankov e K. Pflüger, On a semilinear Schrödinger equation with periodic potential, Nonlinear Analysis, 33 (1998), 593-690.

[37] M. Poppenberg, K. Schmitt e Z. Q. Wang, On the existence of soliton solutions to quasilinear Schrödinger equations. Calc. Var. Partial Differential Equations, 14 (2002), 329-344. 
[38] P. H. Rabinowitz, Some critical point theorems and applications to semilinear elliptic partial differential equations, Ann. Scuola Norm. Sup. Pisa Cl. Sci., 5 (1978), 215-223.

[39] M. Reed e B. Simon, Methods of Mathematical Physics, Volume IV, Academic Press, New York, 1978.

[40] W. Rudin, Real and Complex Analysis, Third Edition, McGrawn-Hill Book Co., 1987.

[41] D. Ruiz e G. Siciliano, Existence of ground states for a nonlinear Schrödinger equation. Nonlinearity, 23 (2010), 1221-1233.

[42] E. A. B. Silva e G. F. Vieira, Quasilinear asymptotically periodic Schrödinger equations with critical growth. Calc. Var., 39 (2010), 1-33.

[43] M. Struwe, A global compactness result for elliptic boundary value problems involving limiting nonlinearities. Math. Z., 187, no. 4, (1984), 511-517.

[44] C. A. Stuart, An introduction to elliptic equation in $\mathbb{R}^{N}$, Trieste Notes, 1998.

[45] C. A. Stuart, Guidance properties of nonlinear planar waveguides, Arch. Rational Mech. Anal., 125 (1993), 145-200.

[46] C. A. Stuart e H. S. Zhou, Applying the mountain pass theorem to an asymptotically linear elliptic equation on $\mathbb{R}^{N}$, Comm. Partial Differential Equations, 24 (1999), $1731-1758$.

[47] A. Szulkin e T. Weth, Ground state solutions for some indefinite variational problems, J. Func. Anal., 257 (2009), 3802-3822.

[48] M. Yang e Y. Ding, Existence of semiclassical states for a quasilinear Schrödinger equation with critical exponent in $\mathbb{R}^{N}$. Ann. Mat. Pura Appl., 192 (2013), 783-804.

[49] J. Zhang, X. Tang e W. Zhang, Infinitely many solutions of quasilinear Schrödinger equation with sign-changing potential. J. Math. Anal. and Appl., 420 (2014), 17621775 .

[50] M. Willem, Minimax Theorems, Volume 24, Birkhauser, Boston, 1996. 